\title{
A Comprehensive Statistically-Based Method to Interpret Real-Time Flowing Measurements
}

\author{
Annual Report
}

Period Start August 2003

Period End August 2004

By

Pinan Dawkrajai, Analis A. Romero, Keita Yoshioka, Dr. Ding Zhu, Dr. A. D. Hill and Dr. Larry W. Lake

Oct 2004

DOE Award Number; DE-FC26-03NT15402

The University of Texas at Austin

Texas A\&M University at College Station 


\section{Disclaimer}

This report was prepared as an account of work sponsored by an agency of the United States Government. Neither the United States Government nor any agency thereof, nor any of their employees makes any warranty, express or implied, or assumes any legal liability or responsibility for the accuracy, that its use would not infringe privately owned rights. Reference herein to any specific commercial product, process, or service by trade name, trademark, manufacturer, or otherwise does not necessarily agency thereof. The views and opinions of authors expressed herein do not necessarily state or reflect those of United States Government or any agency thereof. 


\begin{abstract}
In this project, we are developing new methods for interpreting measurements in complex wells (horizontal, multilateral and multi-branching wells) to determine the profiles of oil, gas, and water entry. These methods are needed to take full advantage of "smart" well instrumentation, a technology that is rapidly evolving to provide the ability to continuously and permanently monitor downhole temperature, pressure, volumetric flow rate, and perhaps other fluid flow properties at many locations along a wellbore; and hence, to control and optimize well performance.

In this first year, we have made considerable progress in the development of the forward model of temperature and pressure behavior in complex wells. In this period, we have progressed on three major parts of the forward problem of predicting the temperature and pressure behavior in complex wells. These three parts are the temperature and pressure behaviors in the reservoir near the wellbore, in the wellbore or laterals in the producing intervals, and in the build sections connecting the laterals, respectively.

Many models exist to predict pressure behavior in reservoirs and wells, but these are almost always isothermal models. To predict temperature behavior we derived general mass, momentum, and energy balance equations for these parts of the complex well system. Analytical solutions for the reservoir and wellbore parts for certain special conditions show the magnitude of thermal effects that could occur. Our preliminary sensitivity analyses show that thermal effects caused by near-wellbore reservoir flow can cause temperature changes that are measurable with smart well technology. This is encouraging for the further development of the inverse model.
\end{abstract}




\section{Table of Contents}

Title Page

Disclaimer

Abstract

Table of Contents

Lists of Graphical Materials

1. Introduction $\quad--1$

2. Executive Summary

3. Reservoir Model

3.1 Background Information

3.2 Derivation of Governing Equations for Reservoir Flow ---7

3.2.1 Mass Balance

3.2.2 Energy Balance

3.3 Temperature Model for Slightly Compressible Flow $\quad$---10

4. Well Model ---13

4.1 Physical Problem Description of Producing Wellbore ---13

4.2 Derivation of Governing Equations

4.2.1 Mass Balance

4.2.2 Momentum Balance $\quad$---15

4.2.3 Energy Balance $\quad$---17

4.2.4 Steady State Equations $\quad---20$

4.3 Model Development

4.3.1 Iterative Numerical Method $\quad$---24 
4.4 Results and Discussion

4.4.1 Example Calculation

4.4.2 Comparisons of Several Cases

4.5 Conclusion of Well Model

5. Model Development of Build Section

5.2 Temperature at Junctions

5.3 Results and Discussion

5.3.2 Temperature Profile along the Build Section and Mixed Zone ---53

Appendix A

Temperature Model for Slightly Compressible Fluid

Appendix B

Temperature Model for Slightly Compressible Fluid in Radial Coordinate

---66

Appendix C

Another Derivation of Governing Equation for Producing Wellbore 


\section{Lists of Graphical Materials}

Table

4.1

Sample values of properties $\quad$---31

4.2

Properties in example calculation $\quad---34$

5.1

Properties used in calculation of temperature profiles

$---53$

5.2

Properties used in calculation of temperature profiles for two

laterals with same heat capacities and flow rates mixed at junction ---55

5.3 Properties used in calculation of temperature profiles along build section and junction with different heat capacities and flow rates ---56

Figure

3.1

3.2

3.3

3.4

4.1

4.2

4.3

4.4

4.5

4.6

4.7

4.8

4.9

4.10

4.11

4.12

4.13

4.14

4.15

4.16

4.17

4.18

4.19

4.20

4.21

4.22

4.23

4.24

4.25

4.26

4.27

5.1
Horizontal well flow geometry in a rectangular reservoir

Isenthalpic flow diagram

Flow region in rectangular reservoir

Temperature of fluid flowing into a horizontal wellbore

Physical system - Wellbore model

Averaged velocities for laminar flow and turbulent flow

Boundary pressure

Cell image

Program flow chart

Flow rate profile with uniform inflow

Generated friction factor value with given flow rate profile

Velocity profile comparison with prediction model

Pressure profile comparison with prediction model

Temperature profile comparison with prediction model

Predicted flow rate profile

Predicted pressure profile

Predicted temperature profile

Inflow rate profile comparison

Flow rate profile comparison

Pressure profile comparison

Temperature profile comparison

Image of outside temperature distribution with damage zone

Inflow profile with damage zone

Flow rate profile with damage zone

Pressure profile with damage zone

Temperature profile with hot region

Production scheme

Inflow profile with production intervals

Flow rate profile with production intervals

Pressure profile with production intervals

Temperature profile with production intervals

Control volume
$---5$

$---8$

$---10$

$---12$

$---13$

$---17$

$---21$

$---24$

$---27$

$---29$

$---32$

$---32$

$---32$

$---33$

$---34$

$---35$

---35

---36

$---37$

$---37$

---38

---39

---39

$---40$

---40

---41

---42

---42

$---43$

---43

---44

$---47$ 
Comparison of predicted temperature profile with constant angles and variable angle along build section 


\section{Introduction}

Intelligent well completions are being increasingly used in complex wells (horizontal, multilateral and multibranching). Intelligent completions supply real time temperature and pressure profile measurements which can be used to determine flow rate profile, phases, reservoir productivity and fluid properties.

The temperature prediction models for vertical well or inclined well have been studied extensively, however there is little work on horizontal wells. Temperature prediction models of vertical wells focus on the conductive heat transfer between the formation and the wellbore. Since in the vertical production system, temperature near surface is significantly different from the temperature at the deep producing zone, dominant heat transfer will be conduction and it is less difficult to describe temperature profile along the vertical well.

Horizontal or nearly horizontal wells are usually surrounded by almost the same formation temperature. In development of a temperature model for this case, overlooking any effect might lead to misunderstanding of the temperature distribution. It is also true for the pressure profile. In horizontal wells, because there is little gravity pressure drop, any other term such as friction or momentum difference will be the dominant terms. In this problem, there exist two flow directions. One direction is the main flow which runs through the wellbore and the other stream is the inflow from the formation that flows in the radial direction. Therefore, the main difference between vertical and horizontal wells is that the equations must explain the effect of inflow.

In this period, we have progressed on three major parts of the forward problem of predicting the temperature and pressure behavior in complex wells. These three parts are the temperature and pressure behaviors in the reservoir near the wellbore, in the wellbore or laterals in the producing intervals, and in the build sections connecting the laterals, respectively. Detailed results for each of these regions are given in the following sections. 


\section{Executive Summary}

In this project, we are developing new methods for interpreting measurements in complex wells (horizontal, multilateral and multi-branching wells) to determine the profiles of oil, gas, and water entry. These methods are needed to take full advantage of "smart" well instrumentation, a technology that is rapidly evolving to provide the ability to continuously and permanently monitor downhole temperature, pressure, volumetric flow rate, and perhaps other fluid flow properties at many locations along a wellbore; and hence, to control and optimize well performance. This spatial and temporal measurement density is unprecedented in the oil industry, and offers the promise of revolutionary changes in the way complex wells are operated. However, the key to realizing the value of smart wells is the efficient and accurate interpretation of the raw data being acquired. Converting this raw information about wellbore conditions into the useful knowledge of the phase flow profiles is the primary goal of this project.

The specific objectives of the project are:

1. Develop a model to predict temperature, pressure, and flow profiles in complex wells, including nominally horizontal laterals, variably-inclined build sections, wellbore junctions, each of which may have commingled fluids with different properties.

2. Develop inverse methods to infer phase flow profiles (the distribution of oil, water, and gas inflow along a complex well) from continuously monitored data.

In this first year, we have made considerable progress in the development of the forward model of temperature and pressure behavior in complex wells. In this period, we have progressed on three major parts of the forward problem of predicting the temperature and pressure behavior in complex wells. These three parts are the temperature and pressure behaviors in the reservoir near the wellbore, in the wellbore or laterals in the producing intervals, and in the build sections connecting the laterals, respectively.

To develop the forward model of reservoir behavior, we began by deriving very general mass and energy balance equations for this system. The unique feature of this model compared with most models of reservoir flow is that it does not assume isothermal conditions. Instead, subtle energy effects that affect temperature including frictional dissipation and Joule-Thomson expansion are included. We obtained analytical solutions to the governing equations for non-isothermal reservoir flow that have been very informative. In particular, they show that larger enough thermal effects caused by flow in the near-well vicinity occur to be detectable with current downhole temperature measurements.

A similar approach was taken to develop a model of temperature and pressure behavior in the producing laterals. General mass, momentum, and energy balance equations were derived to solve for the temperature, pressure, and flow profiles along the wellbore. A numerical solution to these equations was obtained which can be applied to a wide range of well flow conditions. We tested the numerical model against an analytical solution that we obtained for the special condition of constant inflow along the well, and found excellent agreement, validating the numerical model. The wellbore model shows that thermal effects generated by the wellbore flow itself are small, but changes that occur when inflow conditions vary may be detectable. 
To model the temperature in the build sections connecting individual laterals, we adapted the Ramey equation to the condition of a changing wellbore inclination. We then developed energy balance equation applied at the junctions. The combination of these allows us to predict the temperature profile along all build sections, and above the junction locations. This temperature model is now being coupled with a two-phase pressure drop algorithm to obtain both the pressure and temperature profiles in build sections. 


\section{Reservoir Model}

\subsection{Background Information}

Horizontal Inflow Models. Hydrocarbon production by means of horizontal wells has become popular during the past few decades. Generally, horizontal wells are rarely perfectly horizontal; rather, they have many bends and curves with local inclinations over 80 degrees from the vertical. The horizontal length could be several thousands of feet long. Horizontal wells provide larger contact area with the reservoir by increasing the surface area of the wellbore. That means a higher production rate and larger drainage area compared to a vertical well. In addition, more than one horizontal section could be drilled from the same vertical section to recover hydrocarbon from the same or different reservoirs. These benefits draw attention to modeling the flow behavior of fluid into horizontal wells.

There are many isothermal steady-state inflow models for horizontal wells. They are much more complex than vertical well inflow models because the flow is constrained by the horizontal reservoir boundaries and more affected by permeability anisotropy. Certain assumptions must be made to derive an analytical model. Butler (1994) derived a model for a fully-penetrating horizontal well by using conformal mapping. Butler's model for an isotropic reservoir is

$p_{y}-p_{w}=\frac{q \mu}{2 k L h}\left\{|y|+\frac{h}{\pi} \ln \left(\frac{h}{2 \pi r_{w}}\right)\right\} \quad$ for large $|y|$

Gringarten et al. (1973) and Ouyang et al., (1998) used Green's functions (instantaneous source function) to solve the diffusivity equation. The plane, line, and point sources are used with Newman's product method to generate solution for reservoir flow. The solution applies to steady-state flow by using long time approximation $(t \rightarrow \infty)$. For a fully-penetrating horizontal well in rectangular reservoir, the pressure drop can be written as below

$$
\begin{aligned}
& S_{y}=\frac{1}{2 \sqrt{\pi \eta_{y} \tau}} \exp \left[-\frac{\left(y-y_{w}\right)^{2}}{4 \eta_{y} \tau}\right] \\
& S_{z}=\frac{1}{h}\left[1+2 \sum_{n=1}^{\infty} \exp \left(-\frac{n^{2} \pi^{2} \eta_{z} \tau}{h^{2}}\right) \cos \left(\frac{\pi z_{w}}{h}\right) \cos \left(\frac{n \pi z}{h}\right)\right] \\
& \eta_{i}=\frac{k_{i}}{\phi c_{t} \mu}
\end{aligned}
$$

and

$$
\Delta p\left(y, z, y_{w}, z_{w}, t\right)=\frac{q}{\phi c_{t} L} \int_{0}^{t} S_{y} S_{z} d \tau
$$


Symbols are defined in the Nomenclature. The solution is not easy to couple with an energy equation to predict temperature. It is presented here just for a comparison.

A recent inflow model presented by Furui et al. (2003) is based on finite element simulations for a fully penetrating horizontal well. The model is more simple, concise, and easy to couple with an energy balance. The pressure drops in two flow regions (linear and radial flow) are

$\Delta p_{\text {linear }}=\frac{(q / 2) \mu}{k h L}\left(\frac{Y}{2}-y_{t}\right)$

and

$\Delta p_{\text {radial }}=\frac{q \mu}{2 \pi k L} \ln \left(\frac{r_{t}}{r_{w}}\right)$

where $r_{t}=\frac{h}{2} \sqrt{2}$ and $y_{t}=\frac{h}{2}$, the position of $\mathrm{r}_{\mathrm{t}}$ and $\mathrm{y}_{\mathrm{t}}$ are shown on the figure below
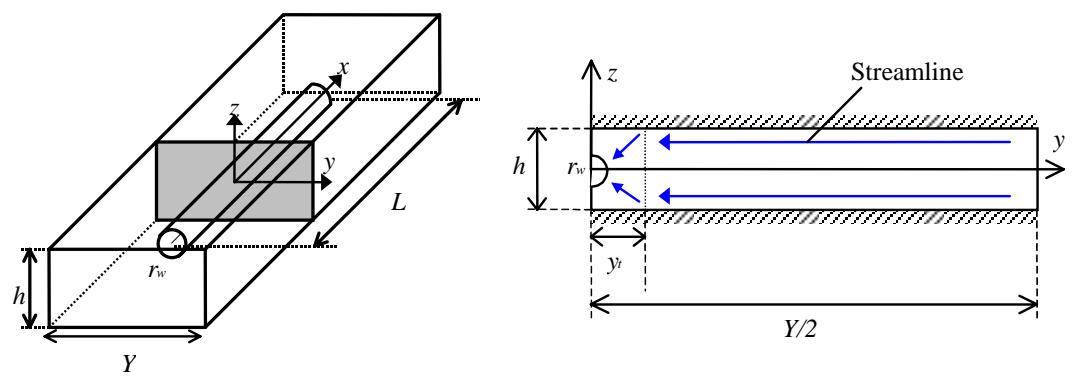

Fig. 3.1 Horizontal well flow geometry in a rectangular reservoir (Furui et. al., 2002)

Temperature Logging. Non-isothermal flow models are usually found in the temperature log interpretation literature (Hill, 1990). It has been recognized that gas entering a wellbore often creates a cooling due to Joule-Thomson expansion and water entry causes the heat increase. The temperature change of fluids can be roughly determined from the Joule-Thomson coefficient $\mathrm{K}_{\mathrm{JT}}$ which is

$$
K_{J T}=\left(\frac{\partial T}{\partial p}\right)_{\hat{H}}=\frac{1}{C_{p}}\left[T\left(\frac{\partial \hat{V}}{\partial T}\right)_{p}-\hat{V}\right]=\frac{\beta T-1}{\rho C_{p}}
$$

Thus, the relationship between the change of temperature and pressure is

$$
\Delta T=\left(\frac{\beta T-1}{\rho C_{p}}\right) \Delta p
$$


for constant $\mathrm{K}_{\mathrm{JT}}$, where $\beta$ is the thermal expansion coefficient defined as $\frac{1}{\hat{V}}\left(\frac{\partial \hat{V}}{\partial T}\right)_{p}$, T is temperature, $\hat{V}$ is specific volume, $\mathrm{C}_{\mathrm{p}}$ is specific heat capacity and $\rho$ is the density of the fluid. For an ideal gas, $\beta=\frac{1}{T}, K_{J T}=0$. For real gases at low pressure, $\beta T>1$, resulting in $K_{J T}>0$. Thus, a pressure drop $(\Delta p<0)$ causes cooling. On the other hand, for liquid flow $\beta T<1, K_{J T}<0$. Therefore, a pressure drop causes heating.

Most studies have focused on developing wellbore models for thermal changes caused by conduction and convection. They also have assumed that the produced fluid enters the wellbore at the geothermal temperature. Steffensen and Smith (1973) recognized the importance of the heating or cooling of the produced fluid before it enters the wellbore and developed models incorporating the Joule-Thomson coefficient. However; flow in permeable media does not fulfill isenthalpic conditions because there is also heat generated by friction between rock matrix and flowing fluids. The amount of frictional heating is greatest near the wellbore where the pressure gradient is the largest (Hill, 1990).

Maubeuge et al. (1994) presented an interesting approach to production logging interpretation. They acknowledged the decompression of the fluid and the frictional heating that occurs in the formation and developed a finite element numerical well model named MOTHER, a 2D radial symmetric single well model. Only a single phase is flowing and its properties are considered constant for the liquids and are calculated by correlation for gases. A standard analytical solution from well testing is used for the pressure distribution in reservoir. The model was tested by matching its results with measurements from dynamic gauges (production logs). The good fits in both pressure and downhole flow rate are obtained. Nevertheless, MOTHER has not yet quantitatively fitted a temperature profile because it underestimates heating in case of an oil producing well. Maubeuge et al. suggested the possibilities of further development by taking into account formation damage in the neighborhood of the well. The energy equation used in MOTHER is

$$
\rho C_{p} \overrightarrow{\mathbf{u}} \cdot \vec{\nabla} T+\overrightarrow{\mathbf{u}} \cdot \vec{\nabla} p-\beta T \overrightarrow{\mathbf{u}} \cdot \vec{\nabla} p-\vec{\nabla} \cdot K_{T t} \vec{\nabla} T=-\left(\rho c_{p}\right)_{\text {total }} \frac{d T}{d t}+\beta T \phi \frac{\partial p}{\partial t}
$$

Equation 3.8 will later be compared with the energy equation presented in Section 3.2.2 of this research.

Distributed Temperature Measurement. Distributed temperature monitoring of downhole conditions in horizontal wells is an advanced measurement technology that can be used to obtain reservoir temperature information. Fiber sensors now provide reliable temperature measurements with resolution less than $0.1{ }^{\circ} \mathrm{C}$. They can provide information at distance of up to $10 \mathrm{~km}$, with a spatial resolution of $1 \mathrm{~m}$, and with a measurement time of typically a few minutes (Sensonet Ltd, 2004). 
Fiber sensors have proven useful in many applications. For example, in an Oman oilfield fiber sensors were installed in several long horizontal open-hole completion intervals of production and injection wells. The results show that it is cost effective and less risky than conventional production logging in horizontal wells. Analysis of the data has helped the understanding of flow in a horizontal producer and injector (Brown et al., 2003). Another application of this technology is to install sensors together with downhole mechanical instrumentation such as valves and inflow control devices. Distributed temperature devices at meter long intervals in the wellbore provide real-time data that help identify water flowing into a particular section. Then, an action to shut in the zone is possible with remotely operated hydraulic interval control values (Tolan et al., 2001).

\subsection{Derivation of Governing Equations for Reservoir Flow}

The fundamental equations describing fluid flow in a reservoir are mass balance, Darcy's law, and energy balance. These equations are very general. They are discussed and formulated to fit the scope of this study.

\subsubsection{Mass Balance}

A starting point for studying fluid flow is the mass balance. It is the conservation of mass per unit area (perpendicular to the velocity vector) per unit time. By understanding the mechanisms of mass flow, we can infer velocity and pressure distribution of fluid in space. The velocity and pressure distribution will then be used in the energy equation.

Lake (1989) formulated a mass balance that can apply directly to fluid flow in permeable media. It is simplified here for steady state flow condition.

$$
\vec{\nabla} \cdot(\rho \overrightarrow{\mathbf{u}})=0
$$

where the auxiliary relation $\overrightarrow{\mathbf{u}}=-\frac{\overrightarrow{\overrightarrow{\mathbf{k}}}}{\mu} \cdot(\vec{\nabla} p+\rho \overrightarrow{\mathbf{g}})$ is derived from Darcy's law.

\subsubsection{Energy Balance}

The law of conservation of energy is an extension of the first law of thermodynamics, which involves the difference in internal energy of two equilibrium states of a closed system because of the heat added to the system and the work done on the system.

$$
\Delta U=\mathrm{Q}+\mathrm{W}
$$

where $\Delta U$ is the differential of the internal energy, $\mathrm{U}$, and $\mathrm{Q}$ and $\mathrm{W}$ are the heat absorbed by the system, and the work done on the system. $\Delta U$ is the differential of the state variable $\mathrm{U}$. Q and $\mathrm{W}$ are not functions of state. 


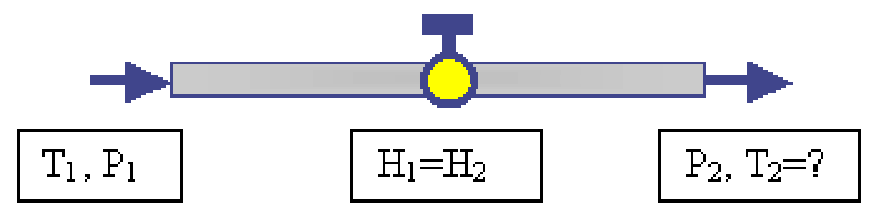

Fig. 3.2 Isenthalpic flow diagram

The Joule-Thomson experiment can be explained by this law. Assume that the throttle valve above is insulated so that no heat is transferred during the process. And imagine this as a closed system with the same amount of gas entering and leaving the valve but both systems have different volumes (just like free expansion). The gas initially has a pressure $P_{1}$, temperature $T_{1}$ and volume $V_{1}$. After it passes through the valve, its pressure is $\mathrm{P}_{2}$ and the volume is $\mathrm{V}_{2}$. If the kinetic and potential energy change of the gas can be neglected, then the first law of this system can be written as $U_{2}$ $\mathrm{U}_{1}=\mathrm{Q}+\mathrm{W}$. By neglecting any shaft work, and the system is insulated $(\mathrm{Q}=0) . \mathrm{W}=\mathrm{P}_{1} \mathrm{~V}_{1^{-}}$ $\mathrm{P}_{2} \mathrm{~V}_{2}$ so that the above equation becomes $\mathrm{U}_{2}+\mathrm{P}_{2} \mathrm{~V}_{2}=\mathrm{U}_{1}+\mathrm{P}_{1} \mathrm{~V}_{1}$ or $\mathrm{H}_{2}=\mathrm{H}_{1}$.

Thus, the Joule-Thomson experiment is an isenthalpic process. The expression for JouleThomson coefficient is shown in Eq. 3.6.

The general form of the conservation of energies is derived rigorously in Bird et al. (2002) and presented here as

$$
\frac{\partial}{\partial t}\left(\frac{1}{2} \rho|\overrightarrow{\mathbf{v}}|^{2}+\rho \hat{U}\right)=-\left(\vec{\nabla} \cdot\left(\frac{1}{2} \rho v^{2}+\rho \hat{U}\right) \overrightarrow{\mathbf{v}}\right)-\vec{\nabla} \cdot \vec{q}-\vec{\nabla} \cdot p \overrightarrow{\mathbf{v}}-\vec{\nabla} \cdot[\overrightarrow{\overline{\boldsymbol{\tau}}} \cdot \overrightarrow{\mathbf{v}}]+\rho(\overrightarrow{\mathbf{v}} \cdot \overrightarrow{\mathbf{g}})
$$

The terms from left to right are (1)rate of increase of energy (2)rate of energy by convection transport (3)energy by heat conduction (4) work done on fluid by pressure forces (5) work done on fluid by viscous forces (6) work done on fluid by gravity forces. To express the energy equation in terms of measurable quantities (P,T, etc.), we can rederive the equation for permeable media starting from the fact that the change in combined energy flux vector is equal to zero for steady-state flow.

$$
\vec{\nabla} \cdot \overrightarrow{\mathbf{e}}=0
$$

Substituting the combined energy flux vector, $\vec{e}$, derived in Bird et al. (2002) which is

$$
\begin{aligned}
& \overrightarrow{\mathbf{e}}=\left(\frac{1}{2} \rho|\overrightarrow{\mathbf{v}}|^{2}+\rho \hat{H}\right) \overrightarrow{\mathbf{v}}+\overrightarrow{\overline{\boldsymbol{\tau}}} \cdot \overrightarrow{\mathbf{v}}-\overrightarrow{\overrightarrow{\mathbf{K}}} \vec{\nabla} T \text {, the equation becomes } \\
& \vec{\nabla} \cdot\left[\left(\frac{1}{2} \rho|\overrightarrow{\mathbf{v}}|^{2}+\rho \hat{H}\right) \overrightarrow{\mathbf{v}}+\overrightarrow{\overline{\boldsymbol{\tau}}} \cdot \overrightarrow{\mathbf{v}}-\overrightarrow{\overrightarrow{\mathbf{K}}} \vec{\nabla} T\right]=0
\end{aligned}
$$


Neglecting the kinetic energy term $\left(\frac{1}{2} \rho|\overrightarrow{\mathbf{v}}|^{2}\right)$ and knowing from mass balance that $\rho \overrightarrow{\mathbf{v}}$ is constant, the equation becomes $\rho \overrightarrow{\mathbf{v}} \cdot \vec{\nabla} \hat{H}+\vec{\nabla} \cdot(\overrightarrow{\overline{\boldsymbol{\tau}}} \cdot \overrightarrow{\mathbf{v}})-\vec{\nabla} \cdot(\overrightarrow{\mathbf{K}} \vec{\nabla} T)=0$. The enthalpy $\hat{H}$ can be expanded using a thermodynamic relationship

$$
\begin{aligned}
& \rho \overrightarrow{\mathbf{v}} \cdot\left\{\left[\left(\frac{\partial \hat{H}}{\partial p}\right)_{T} \vec{\nabla} p+\left(\frac{\partial \hat{H}}{\partial T}\right)_{p} \vec{\nabla} T\right]\right\}+\vec{\nabla} \cdot(\overrightarrow{\overline{\boldsymbol{\tau}}} \cdot \overrightarrow{\mathbf{v}})-\overrightarrow{\overrightarrow{\mathbf{K}}} \vec{\nabla} T \\
& =\rho \overrightarrow{\mathbf{v}} \cdot\left\{\left[\hat{V}-T\left(\frac{\partial \hat{V}}{\partial T}\right)_{p}\right] \vec{\nabla} p+C_{p} \vec{\nabla} T\right\}+\vec{\nabla} \cdot(\overrightarrow{\overline{\boldsymbol{\tau}}} \cdot \overrightarrow{\mathbf{v}})-\overrightarrow{\overrightarrow{\mathbf{K}}} \vec{\nabla} T \\
& =\rho \overrightarrow{\mathbf{v}} \cdot\left\{\left[\frac{1}{\rho}-\frac{\beta T}{\rho}\right] \vec{\nabla} p+C_{p} \vec{\nabla} T\right\}+\vec{\nabla} \cdot(\overrightarrow{\overline{\boldsymbol{\tau}}} \cdot \overrightarrow{\mathbf{v}})-\overrightarrow{\overrightarrow{\mathbf{K}}} \vec{\nabla} T \\
& =\overrightarrow{\mathbf{v}} \cdot \vec{\nabla} p-\beta T \overrightarrow{\mathbf{v}} \vec{\nabla} p+\rho C_{p} \overrightarrow{\mathbf{v}} \vec{\nabla} T+\vec{\nabla} \cdot(\overrightarrow{\vec{\tau}} \cdot \overrightarrow{\mathbf{v}})-\overrightarrow{\overrightarrow{\mathbf{K}}} \vec{\nabla} T=0
\end{aligned}
$$

The $\overrightarrow{\overrightarrow{\boldsymbol{\tau}}} \cdot \overrightarrow{\mathbf{v}}$ is frictional energy that converted from mechanical work (sometimes called the viscous dissipation heating). Using the fact that $\tau_{x y}=\tau_{x z}=\tau_{y z}=0$, the work done by the frictional forces is given by $\vec{\nabla} \cdot(\overrightarrow{\overrightarrow{\boldsymbol{\tau}}} \cdot \overrightarrow{\mathbf{v}})=\frac{\partial}{\partial x}\left(\tau_{x} v_{x}\right)+\frac{\partial}{\partial y}\left(\tau_{y} v_{y}\right)+\frac{\partial}{\partial z}\left(\tau_{z} v_{z}\right)$. Introducing the constitutive equation $\left(\tau_{x}=\tau_{y}=\tau_{z}=p\right)$, the equation is simplified to $\vec{\nabla} \cdot(\overrightarrow{\boldsymbol{\tau}} \cdot \overrightarrow{\mathbf{v}})=\vec{\nabla} \cdot(p \overrightarrow{\mathbf{v}})$. The work done by the frictional forces is commonly represented by $\vec{\nabla} \cdot(p \overrightarrow{\mathbf{v}})$, see Ingham et al. (1990) and Al-Hadhrami et al. (2002).

In permeable media, the velocity in $\mathrm{x}$-direction, $\vec{v}$ is replaced by superficial velocity, $\frac{\overrightarrow{\mathbf{u}}}{\phi}$. And, the heat conduction term, $\overrightarrow{\mathbf{K}} \vec{\nabla} T$, is converted to effective heat conduction which combines both fluid and matrix. Then, the equation becomes

$$
\rho C_{p} \overrightarrow{\mathbf{u}} \cdot \vec{\nabla} T+\overrightarrow{\mathbf{u}} \cdot \vec{\nabla} p-\beta T \overrightarrow{\mathbf{u}} \cdot \vec{\nabla} p+\vec{\nabla} \cdot(p \overrightarrow{\mathbf{u}})-\vec{\nabla} \cdot \overrightarrow{\mathbf{K}}_{T t} \vec{\nabla} T=0
$$

The first three terms combined describes the Joule-Thomson effect which includes convection transport, work done on fluid by pressure forces (heating), and thermal expansion (cooling). The fourth term stands for the frictional heating. The last term is effective heat conduction which combines both fluid and matrix conduction.

If we were to use the energy equation (Eq. 3.13) to describe the Joule-Thomson experiment, which is a steady state isenthalpic process with no heat conduction and frictional heating terms, we would arrive at $\rho C_{p} \overrightarrow{\mathbf{u}} \cdot \vec{\nabla} T+\overrightarrow{\mathbf{u}} \cdot \vec{\nabla} p-\beta T \overrightarrow{\mathbf{u}} \cdot \vec{\nabla} p=0$ In one dimensional flow (x-direction), the equation become 
$\rho C_{p} u_{x} \frac{d T}{d x}+u_{x} \frac{d p}{d x}-\beta T u_{x} \frac{d p}{d x}=0 \quad$, which can be rearranged as $\Delta T=\left(\frac{\beta T-1}{\rho C_{p}}\right) \Delta p$

The term $\frac{\beta T-1}{\rho C_{p}}$ is the Joule-Thomson coefficient, $\mathrm{K}_{\mathrm{JT}}$. This is a well-known relationship that describes the change in temperature of a fluid upon expansion in a steady state flow with neither heat nor work done on the system. An example of this kind of process is a flow through an expansion valve.

\subsection{A Temperature Model for Slightly Compressible Fluid}

Consider a horizontal well fully penetrated through a rectangular homogeneous reservoir with no-flow boundaries at the top and bottom of the reservoir as shown in the figure below. Flow in the reservoir is in the y-direction and the z-direction, the $\mathrm{x}$ direction is the horizontal wellbore direction.

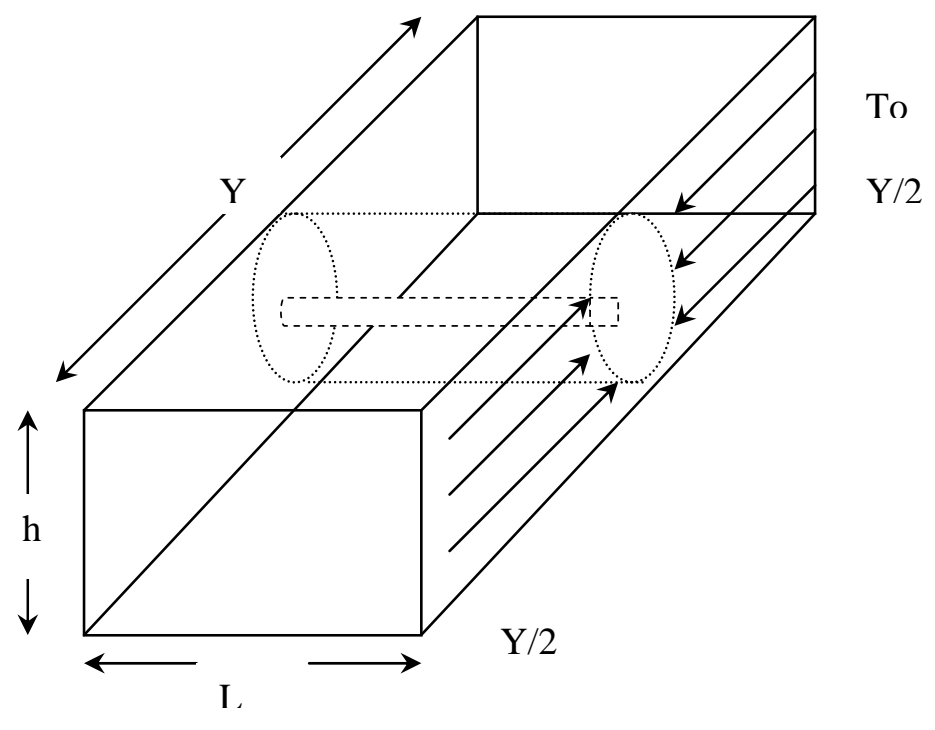

Fig. 3.3 Flow region in rectangular reservoir

For steady state-flow, there are two flow regions (radial and linear) if the transitional flow region between linear and radial flow is neglected. Proper boundary conditions for this model are

1) $T=T_{o}$ at $y=\frac{Y}{2}$

2) $T$ is finite as $r$ approaches zero 
3) $\left(\frac{\partial T}{\partial r}\right)_{r \rightarrow h / 2}=\left(\frac{\partial T}{\partial y}\right)_{y \rightarrow h / 2}$

4) $T_{r \rightarrow h / 2}=T_{y \rightarrow h / 2}$

With these boundary conditions, mass and energy balances discussed earlier can be solved analytically, and the temperature of the fluid entering the horizontal wellbore is obtained. (See Appendix A for a detailed derivation)

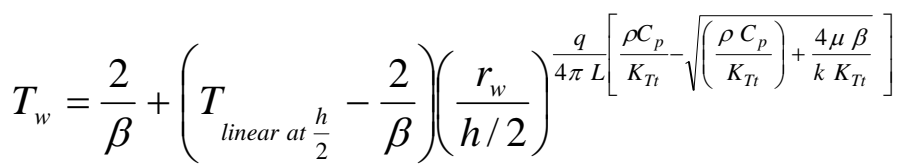

Where

$$
\begin{aligned}
& T_{\text {linear } a t \frac{h}{2}}=c_{1} e^{m_{1} h / 2}+c_{2} e^{m_{2} h / 2}+\frac{2}{\beta} \\
& m_{1}=\frac{q}{4 h L}\left[\frac{\rho C_{p}}{K_{T t}}+\sqrt{\left(\frac{\rho C_{p}}{K_{T t}}\right)^{2}+\frac{4 \beta \mu}{k K_{T t}}}\right] \\
& m_{2}=\frac{q}{4 h L}\left[\frac{\rho C_{p}}{K_{T t}}-\sqrt{\left(\frac{\rho C_{p}}{K_{T t}}\right)^{2}+\frac{4 \beta \mu}{k K_{T t}}}\right] \\
& c_{1}=\frac{(2 / h) m_{r}\left(T_{e}-2 / \beta\right) e^{m_{2} Y / 2}-\left(T_{o}-2 / \beta\right) m_{2} e^{m_{2} h / 2}}{m_{1} e^{m_{1} h / 2+m_{2} Y / 2}-m_{2} e^{m_{2} h / 2+m_{1} Y / 2}} \\
& C_{2}=\frac{\left(T_{o}-2 / \beta\right) m_{1} e^{m_{1} h / 2}-(2 / h) m_{r}\left(T_{e}-2 / \beta\right) e^{m_{1} Y / 2}}{m_{1} e^{m_{1} h / 2+m_{2} Y / 2}-m_{2} e^{m_{2} h / 2+m_{1} Y / 2}}
\end{aligned}
$$

To visualize the result, we can insert some typical parameters and plot the temperature distribution in the reservoir as below.

$\mathrm{q}=500 \mathrm{bbl} /$ day, $1,000 \mathrm{bbl} /$ day, $1,500 \mathrm{bbl} /$ day, and 2,000 bbl/day

$\mathrm{Y}=8,100$ feet

$\mathrm{r}_{\mathrm{w}}=0.5$ feet

$\mathrm{h}=100$ feet

$\mathrm{L}=1,000$ feet 
$\mathrm{k}=200 \mathrm{md}$

$\mathrm{C}_{\mathrm{p}}=0.52802 \mathrm{BTU} /\left(\mathrm{lbm}^{\circ} \mathrm{F}\right)$

Viscosity $=1.7 \mathrm{cp}$

Density $=50 \mathrm{lb} / \mathrm{ft}^{3}$

$\mathrm{K}_{\mathrm{Tt}}=2 \quad \mathrm{BTU} /\left(\mathrm{hr} \mathrm{ft}^{\circ} \mathrm{F}\right)$

$\mathrm{T}_{0}=180{ }^{\circ} \mathrm{F}$

$\beta=0.0005761 /{ }^{\circ} \mathrm{F}$

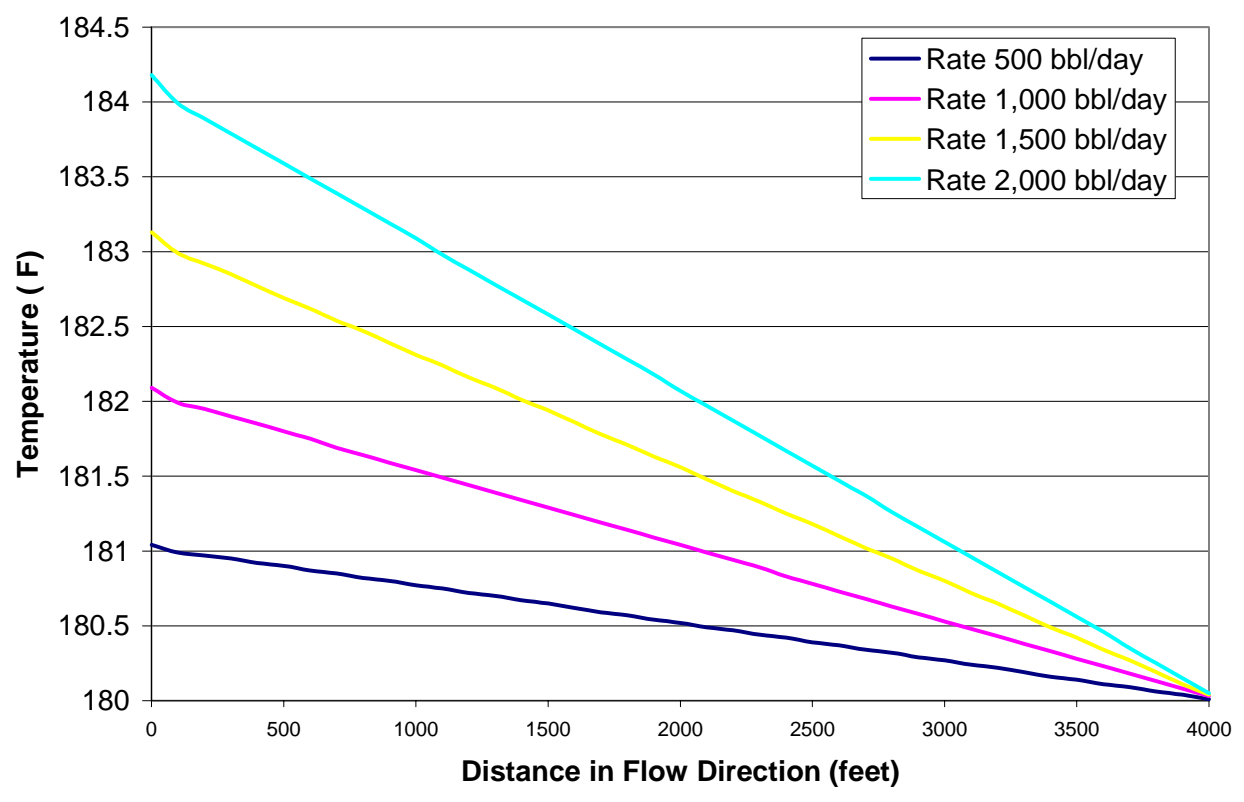

Fig. 3.4 Temperature of fluid flowing into a horizontal wellbore

As shown in the model (Fig. 3.4), the temperature of fluid entering a well $\left(T_{w}\right)$ depends on flow rate, drawdown pressure, type of fluid, and reservoir properties. By varying these parameters, we would be able to match the measured temperature in a similar manner as history matching. That means this analytical model must be coupled with a wellbore model together with multisegment technique to obtain temperature distribution along a horizontal wellbore. 


\section{Well Model}

\subsection{Physical Problem Description of Producing Wellbore}

In development of the forward model, our objective is that given inflow rate information such as productivity index and reservoir pressure or inflow rate itself, to predict the temperature and pressure profile. Of course pressure profile will be used to know flow rate and temperature distribution. Therefore, we need to estimate the three unknowns that are flow rate, pressure and temperature along the wellbore with or without inflow. The physical system is shown in Fig. 4.1.

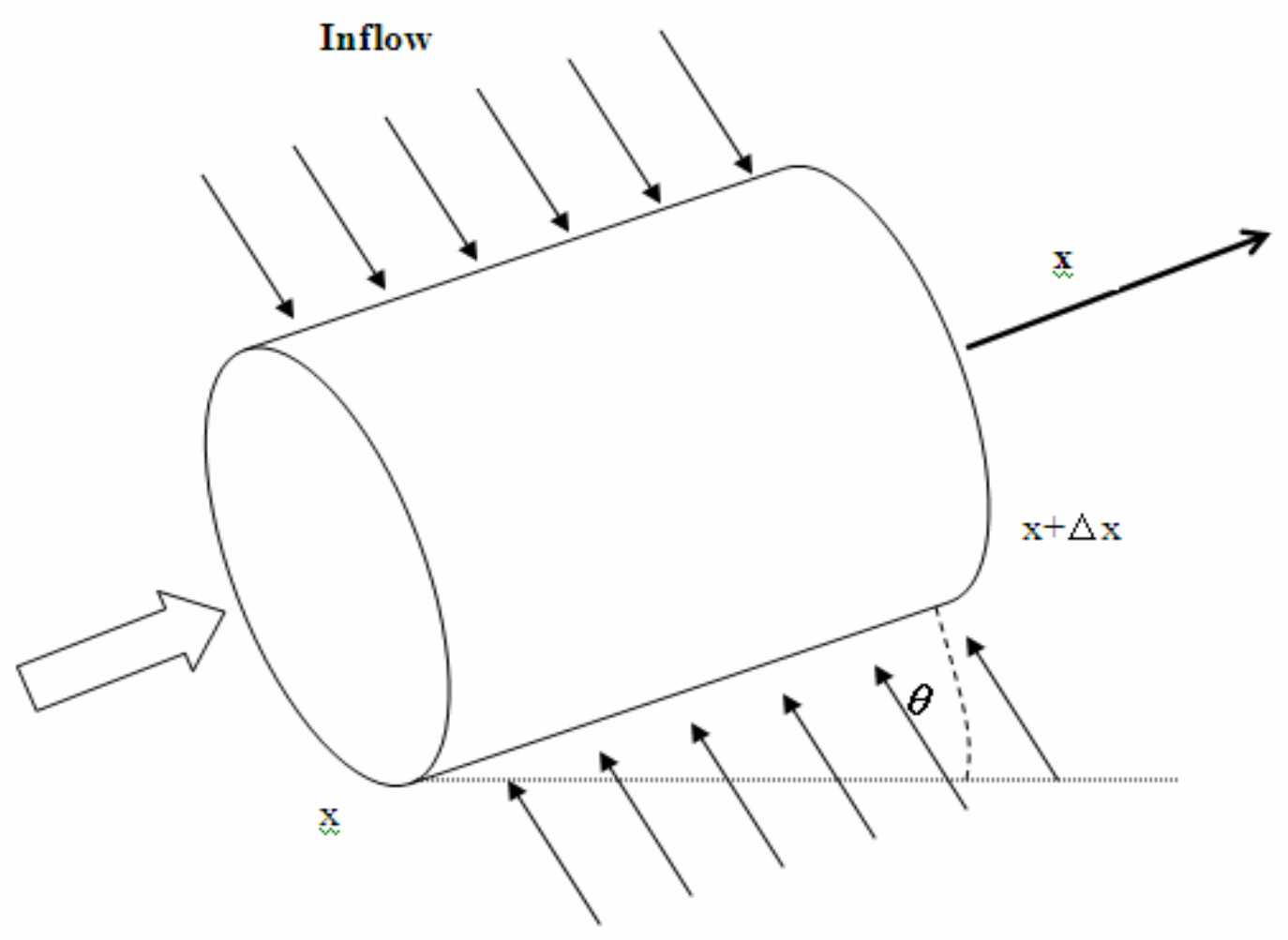

Fig. 4.1 Physical system - Wellbore model

\subsection{Derivation of Governing Equations}

Since we have three unknowns, three equations will be necessary which should be mass, momentum and energy balance equation. In this problem, there are two different velocities. The main stream is flowing through the wellbore and the other is the inflow from the environment (reservoir). Derivation of the governing equations has been done in one dimension following a macroscopic method. 
- Properties are considered to be averaged in cross section.

- Averaged product can be product of averaged component e.g. $\overline{\rho v}=\bar{\rho} \bar{v}$.

- Area averaged velocity is defined in microscopic coordinate as

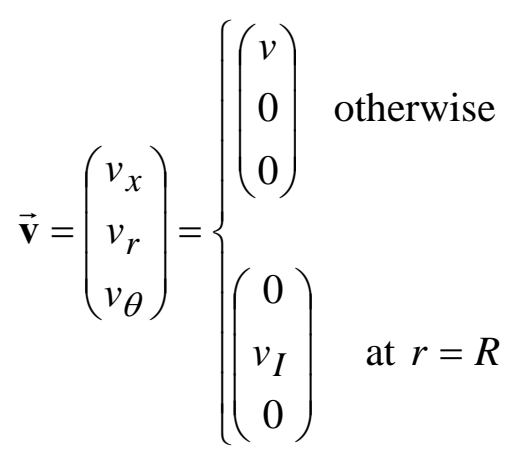

- Eddy flow is neglected and axial velocity is averaged in entire cross sectional area except wall boundary. At the wall boundary velocity is considered only in the radial direction.

\subsubsection{Mass Balance}

Total mass in at $r=R$ is

$\left.\left(\rho v_{r}\right)\right|_{R} 2 \pi R \Delta x$

Total mass in at $x=x$ is

$(\rho v)_{x} \pi R^{2}$

Total mass in at $x=x+\Delta x$ is

$(\rho v)_{x+\Delta x} \pi R^{2}$

Accumulated mass over $\Delta t$ in $\mathrm{CV}$ can be expressed

$\left(\left.\rho\right|_{t=t}-\left.\rho\right|_{t=t+\Delta t}\right) \pi R^{2} \Delta x$

Equating these terms

$\left(\left.\rho\right|_{t=t}-\left.\rho\right|_{t=t+\Delta t}\right) \pi R^{2} \Delta x=\left(\left.\left(\rho v_{r}\right)\right|_{R} 2 \pi R \Delta x+\rho v_{x} \pi R^{2}-\rho v_{x+\Delta x} \pi R^{2}\right) \Delta t$

or 


$$
\frac{\left(\left.\rho\right|_{t=t}-\left.\rho\right|_{t=t+\Delta t}\right)}{\Delta t}=\frac{\rho v_{X}-\rho v_{X+\Delta x}}{\Delta x}+\left.\frac{2}{R}\left(\rho v_{r}\right)\right|_{R}
$$

Taking $\Delta x \rightarrow 0, \Delta t \rightarrow 0$ yields

$\frac{\partial \rho}{\partial t}=-\frac{\partial\left(\rho v_{X}\right)}{\partial x}+\left.\frac{2}{R}\left(\rho v_{r}\right)\right|_{R}$

From the assumption of velocity (Eq. 4.1), it becomes

$$
\frac{\partial \rho}{\partial t}=-\frac{\partial(\rho v)}{\partial x}+\frac{2}{R} \rho v_{I}
$$

\subsubsection{Momentum Balance}

We only need to consider momentum balance in the axial direction not in the radial direction.

Momentum on the surface at $r=R$ is

$\left(\rho v_{r} \cdot v_{x}-\tau_{r x}\right)_{R} 2 \pi R \Delta x$

Now we assume inflow is perpendicular to the axial direction (or from no slip $\left.v_{x}\right|_{R}=0$ assumption). Then, it becomes

$\left(\rho v_{r} \cdot v_{x}-\tau_{r x}\right)_{R} 2 \pi R \Delta x=\left(0-\tau_{r x}\right)_{R} 2 \pi R \Delta x=-\left(\tau_{r x}\right)_{R} 2 \pi R \Delta x$

Momentum at $x=x$ is

$\left(\rho v_{X} \cdot v_{X}+p-\tau_{x X}\right)_{X} \pi R^{2}$

Momentum on $x=x+\Delta x$ is

$\left(\rho v_{X} \cdot v_{X}+p-\tau_{X X}\right)_{X+\Delta x} \pi R^{2}$

Gravity force is given by

$\rho g \sin \theta \pi R^{2} \Delta x$

Accumulated momentum over $\Delta t$ in $\mathrm{CV}$

$\left(\left(\rho v_{x}\right)_{t=t}-\left(\rho v_{x}\right)_{t=t+\Delta t}\right) \pi R^{2} \Delta x$ 
Applying macroscopic condition to stress tensors derived by Navier and Stokes,

The stress tensor in the $x-x$ direction is

$$
\tau_{x x}=2 \mu \frac{\partial v_{x}}{\partial x}-\frac{2}{3} \mu\left[\frac{1}{r} \frac{\partial\left(r v_{r}\right)}{\partial r}+\frac{\partial v_{x}}{\partial x}\right]=2 \mu \frac{\partial v_{x}}{\partial x}-\frac{2}{3} \mu\left[0+\frac{\partial v_{x}}{\partial x}\right]=\frac{4}{3} \mu \frac{\partial v_{x}}{\partial x}
$$

Stress tensor in $x-r$ direction is defined as

$$
\left(\tau_{r x}\right)_{R}=\tau_{w}=\frac{\rho f v_{x}^{2}}{2}
$$

Stress tensor in $r-r$ direction is intuitively

$\tau_{r r}=0$

Equating momentum yields

$$
\begin{aligned}
& \left(\left(\rho v_{x}\right)_{t=t}-\left(\rho v_{x}\right)_{t=t+\Delta t}\right) \pi R^{2} \Delta x \\
& =\left(-\left(\tau_{r x}\right)_{R} 2 \pi R \Delta x+\left\{\left(\rho v_{x} \cdot v_{x}+p-\tau_{x x}\right)_{x}-\left(\rho v_{x} \cdot v_{x}+p-\tau_{x x}\right)_{x+\Delta x}-\rho g \sin \theta\right\} \pi R^{2}\right) \Delta t
\end{aligned}
$$

or

$$
\begin{aligned}
& \frac{\left.\left(\rho v_{x}\right)_{t=t}-\left(\rho v_{x}\right)_{t=t+\Delta t}\right)}{\Delta t}= \\
& -\tau_{w} \frac{2}{R}+\frac{\left(\rho v_{X} \cdot v_{X}+p-\tau_{x X}\right)_{X}-\left(\rho v_{X} \cdot v_{X}+p-\tau_{X X}\right)_{X+\Delta x}}{\Delta x}-\rho g \sin \theta
\end{aligned}
$$

Taking $\Delta x \rightarrow 0, \Delta t \rightarrow 0$, we have

$$
\frac{\partial\left(\rho v_{x}\right)}{\partial t}=-\tau_{w} \frac{2}{R}-\frac{\partial\left(\rho v_{x} \cdot v_{x}+p-\tau_{x x}\right)}{\partial x}-\rho g \sin \theta
$$

We have averaged the velocity in cross sectional area, the image of the averaged velocities are shown in Fig. 4.2. The momentum correction factor $\alpha$ for One-D area averaged velocity is suggested by White that is

$$
\alpha=\frac{1}{\pi R^{2}} \int_{0}^{2 \pi} \int_{0}^{R}\left(\frac{v_{x}}{v}\right) d r d \theta \quad \begin{array}{ll}
\text { For laminar flow } & \alpha=1.3333 \\
& \text { For laminar flow } \quad \alpha=1.013 \sim 1.037
\end{array}
$$




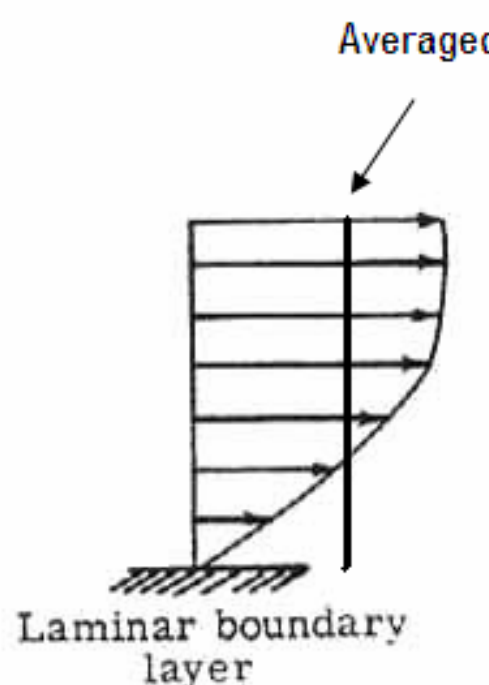

layer

\section{Turbulent boundary}

layer

Fig. 4.2 Averaged velocities for laminar flow and turbulent flow

Taking into account this correction factor and averaged velocity, the momentum balance equation becomes

$$
\frac{\partial(\rho v)}{\partial t}=-\frac{\rho v^{2} f}{R}-\frac{\partial\left(\alpha \rho v^{2}+p\right)}{\partial x}+\frac{\partial}{\partial x}\left(\frac{4}{3} \mu \frac{\partial v}{\partial x}\right)-\rho g \sin \theta
$$

\subsubsection{Energy Balance}

The combined energy flux vector defined by Bird et al. is

$$
\begin{aligned}
\overrightarrow{\mathbf{e}} & =\left(\frac{1}{2} \rho v^{2}+\rho \hat{U}\right) \overrightarrow{\mathbf{v}}+[\overrightarrow{\overline{\boldsymbol{\pi}}} \cdot \overrightarrow{\mathbf{v}}]+\overrightarrow{\mathbf{q}} \\
& =\left(\frac{1}{2} \rho v^{2}+\rho \hat{H}\right) \overrightarrow{\mathbf{v}}+[\overline{\overline{\boldsymbol{\tau}}} \cdot \overrightarrow{\mathbf{v}}]+\overrightarrow{\mathbf{q}}
\end{aligned}
$$

Where

$\overrightarrow{\boldsymbol{\pi}}=p \boldsymbol{\delta}+\overrightarrow{\overline{\boldsymbol{\tau}}}$

Total energy in at $r=R$

$\left.e_{r}\right|_{R} 2 \pi R \Delta x$

Total energy in at $x=x$ 
$\left.e_{x}\right|_{x} \pi R^{2}$

Total energy out at $x=x+\Delta x$

$\left.e_{x}\right|_{x+\Delta x} \pi R^{2}$

Work done by gravity

$\rho v g \sin \theta \pi R^{2}$

Let total accumulative energy be

$\left(\frac{1}{2} \rho v^{2}+\hat{U}\right)=E_{t}$

Total energy in the control volume $(\mathrm{CV})$ is

$\left(\frac{1}{2} \rho v^{2}+\hat{U}\right) \pi R^{2} \Delta x=E_{t} \pi R^{2} \Delta x$

Then accumulated energy over $\Delta t$ in $\mathrm{CV}$ becomes

$\left(\left.E_{t}\right|_{t}-\left.E_{t}\right|_{t+\Delta t}\right) \pi R^{2} \Delta x$

The energy balance equation becomes

$\left(\left.E_{t}\right|_{t}-\left.E_{t}\right|_{t+\Delta t}\right) \pi R^{2} \Delta x$

$=\left(\left.e_{r}\right|_{R} 2 \pi R \Delta x+\left.e_{x}\right|_{X} \pi R^{2}-\left.e_{x}\right|_{X+\Delta x} \pi R^{2}+\rho v g \sin \theta \pi R^{2} \Delta x\right) \Delta t$

or

$\frac{\left(\left.E_{t}\right|_{t}-\left.E_{t}\right|_{t+\Delta t}\right)}{\Delta t}=\left.e_{r}\right|_{R} \frac{2}{R}+\frac{\left.e_{x}\right|_{x}-\left.e_{x}\right|_{x+\Delta x}}{\Delta x}+\rho v g \sin \theta$

Taking $\Delta x \rightarrow 0, \Delta t \rightarrow 0$

$\frac{\partial E_{t}}{\partial t}=\left.e_{r}\right|_{R} \frac{2}{R}-\frac{\partial e_{x}}{\partial x}+\rho v g \sin \theta$

Now, we have 


$$
\begin{aligned}
& \left.e_{r}\right|_{R}=\left[\left(\frac{1}{2} \rho v^{2}+\rho \hat{H}\right) v_{r}\right]_{R}+\left.q_{r}\right|_{R}-\left.\left(\tau_{r X} \cdot v_{X}\right)\right|_{R}-\left.\left(\tau_{r r} \cdot v_{r}\right)\right|_{R} \\
& =\left[\left(E_{t}+p\right) v_{r}\right]_{R}-\left.q_{r}\right|_{R}-\left.\left(\tau_{r x} \cdot v_{X}\right)\right|_{R}-0
\end{aligned}
$$

From the assumption,

$$
\left.\left(\tau_{r x} v_{x}\right)\right|_{R}=\tau_{w} \cdot v_{I}=0
$$

Noting that $\left.A\right|_{R}=A_{I}$

$$
\left.e_{r}\right|_{R}=\left(\left(E_{t}\right)_{I}+p_{I}\right) v_{I}+q_{I}
$$

Also

$$
\begin{aligned}
e_{x} & =\left(\frac{1}{2} \rho v^{2}+\hat{H}\right) v_{x}-\tau_{x x} \cdot v_{x}-\tau_{x r} \cdot v_{r}+q_{x} \\
& =\left(E_{t}+p\right) v_{x}-\frac{4}{3} \mu \frac{\partial v_{x}}{\partial x} v_{x}-\tau_{x r} \cdot v_{r}+q_{x}
\end{aligned}
$$

$\tau_{x r}$ is only defined at the wall ( $r=R$ ) though, $v_{r}$ is zero other than the wall. Also at the wall, radial velocity $\left(v_{r}\right)_{R}=v_{I}$ is perpendicular to $\tau_{w}$ direction. Therefore $\tau_{x r} \cdot v_{r}=0$. Applying averaged velocity, we have

$e_{x}=\left(E_{t}+p\right) v-\frac{4}{3} \mu \frac{\partial v}{\partial x} v+q_{x}$

Substituting Eqs. 4.19 and 4.21 into Eq. 4.17 gives

$$
\frac{\partial E_{t}}{\partial t}=-\frac{\partial}{\partial x}\left(\left(E_{t}+p-\frac{4}{3} \mu \frac{\partial v}{\partial x}\right) v+q_{x}\right)+\frac{2}{R}\left[\left(\left(E_{t}\right)_{I}+p_{I}\right) v_{I}+q_{I}\right]+\rho v g \sin \theta
$$

To estimate $E_{t}$

$$
\begin{aligned}
E_{t} & =\rho\left(\frac{1}{2} v^{2}+\hat{U}\right)=\rho\left(\frac{1}{2} v^{2}+\hat{U}+\frac{p}{\rho}\right)-p=\rho\left(\frac{1}{2} v^{2}+\hat{H}\right)-p \\
& =\rho\left(\frac{1}{2} v^{2}+H^{0}+\int_{T^{0}}^{T} C_{p} d T+\int_{p^{0}}^{p}\left[\frac{1}{\rho}-\frac{\beta T}{\rho}\right] d p\right)-p
\end{aligned}
$$

Considering small change from $H^{0}$ 


$$
E_{t}=\rho\left(\frac{1}{2} v^{2}+H^{0}\right)+\rho C_{p}\left(T-T^{0}\right)+(1-\beta T)\left(p-p^{0}\right)-p
$$

or

$$
E_{t}+p=\rho\left(\frac{1}{2} v^{2}+H^{0}\right)+\rho C_{p}\left(T-T^{0}\right)+(1-\beta T)\left(p-p^{0}\right)
$$

Where $\left(E_{t}\right)_{I}$ will be

$$
\left(E_{t}\right)_{I}=\rho\left(\frac{1}{2} v_{I}^{2}+H^{0}\right)+\rho C_{p}\left(T_{I}-T^{0}\right)+(1-\beta T)\left(p_{I}-p^{0}\right)-p_{I}
$$

or

$$
\left(E_{t}\right)_{I}+p_{I}=\rho\left(\frac{1}{2} v_{I}^{2}+H^{0}\right)+\rho C_{p}\left(T_{I}-T^{0}\right)+(1-\beta T)\left(p_{I}-p^{0}\right)
$$

We can also obtain same results by integrating Two-D cylindrical equations for pipe flow. It is shown in Appendix C.

\subsubsection{Steady State Equations}

The profile in the wellbore is determined by the environmental (reservoir) condition. We have derived unsteady state equations as a general form though it takes only a few seconds to minutes to get a steady state condition in the wellbore. Considering the time scale in reservoir, we can say the wellbore flow is always in steady state.

For steady state, the mass balance equation becomes

$$
\frac{d(\rho v)}{d x}=\frac{2}{R}\left(\rho_{I} v_{I}\right)
$$

The momentum balance equation is

$\frac{d p}{d x}=-\frac{\rho v^{2} f}{R}-\alpha \frac{d\left(\rho v^{2}\right)}{d x}-\rho g \sin \theta$

After substitution of mass balance, we obtain

$$
\frac{d p}{d x}=-\frac{\rho v^{2} f}{R}-\alpha\left(\frac{2}{R} \rho_{I} v_{I} v+\rho v \frac{d v}{d x}\right)-\rho g \sin \theta
$$

The energy balance becomes

$$
0=-\frac{d}{d x}\left(\left(E_{t}+p-\frac{4}{3} \mu \frac{\partial v}{\partial x}\right) v+q_{x}\right)+\frac{2}{R}\left[\left(\left(E_{t}\right)_{I}+p_{I}\right) v_{I}+q_{I}\right]+\rho v g \sin \theta
$$


Substituting Eqs. 4.24 and 4.25 yields

$0=-\frac{d}{d x}\left(\left(\frac{1}{2} \rho v^{2}+\rho \hat{H}-\frac{4}{3} \mu \frac{\partial v}{\partial x}\right) v+q_{x}\right)+\frac{2}{R}\left[\left(\frac{1}{2} \rho_{I} v_{I}^{2}+\rho_{I} \hat{H}_{I}\right) v_{I}+q_{I}\right]-\rho v g \sin \theta$

Where

$\frac{d}{d x}\left(\frac{1}{2} \rho v^{3}\right)=\frac{1}{2} v^{2} \frac{d(\rho v)}{d x}+\rho v \frac{d}{d x}\left(\frac{1}{2} v^{2}\right)$

From mass balance (Eq. 4.26)

$\frac{1}{2} v^{2} \frac{d(\rho v)}{d x}+\rho v \frac{d}{d x}\left(\frac{1}{2} v^{2}\right)=\frac{1}{2} v^{2} \frac{2}{R} \rho_{I} v_{I}+\rho v^{2} \frac{d v}{d x}=\frac{1}{R} \rho_{I} v_{I} v^{2}+\rho v^{2} \frac{d v}{d x}$

Similarly,

$\frac{d(\rho \hat{H} v)}{d x}=\rho v \frac{d \hat{H}}{d x}+\hat{H} \frac{d(\rho v)}{d x}=\rho v \frac{d \hat{H}}{d x}+\hat{H} \frac{2}{R} \rho_{I} v_{I}$

Substitution gives

$$
\begin{aligned}
& \frac{\rho_{I} v_{I}}{R}\left(v_{I}^{2}-v^{2}\right)-\rho v^{2} \frac{d v}{d x}-\rho v \frac{d \hat{H}}{d x}+\frac{2}{R} \rho_{I} v_{I}\left(\hat{H}_{I}-\hat{H}\right) \\
& +\frac{4}{3} \mu v \frac{d v}{d x}-\frac{d q_{x}}{d x}+\frac{2}{R} q_{I}-\rho v g \sin \theta=0
\end{aligned}
$$

Let's think about the boundary pressure, $p_{I}$, shown on the figure below.

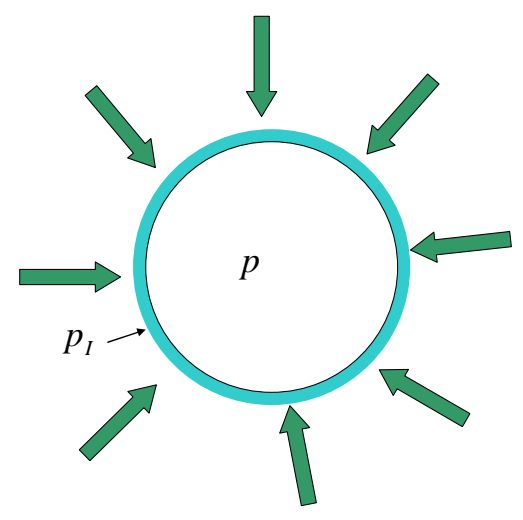

Fig. 4.3 Boundary pressure 
The boundary conditions are given at just outside of the wellbore, inflow is driven by the difference between wellbore pressure and reservoir pressure. So the pressure at the pipe surface can be assumed to be the same as the wellbore pressure. Then, we have

$\rho_{I}=\rho$

The enthalpy difference is

$\hat{H}_{I}-\hat{H}=C_{p}\left(T_{I}-T\right)+\frac{1}{\rho}\left(1-\beta T_{I}\right)\left(p_{I}-p\right)=C_{p}\left(T_{I}-T\right)$

Also, a small enthalpy difference is given as

$d \hat{H}=C_{p} d T+\frac{1}{\rho}\left(1-\beta T_{I}\right) d p$

Finally, the energy balance equation is

$$
\begin{aligned}
& -\rho v C_{p} \frac{d T}{d x}-v(1-\beta T) \frac{d p}{d x}-\rho v^{2} \frac{d v}{d x}+\frac{4}{3} \mu \frac{\partial v}{\partial x}-\frac{d q_{x}}{d x}+\frac{\rho v_{I}}{R}\left(v_{I}^{2}-v^{2}\right) \\
& +\frac{2}{R} \rho v_{I} C_{p}\left(T_{I}-T\right)+\frac{2}{R} q_{I}-\rho v g \sin \theta=0
\end{aligned}
$$

Viscous shear stress between fluids and heat flux between fluids are too small to take into account. Then we have

$$
\begin{aligned}
& -\rho v C_{p} \frac{d T}{d x}-v(1-\beta T) \frac{d p}{d x}-\rho v^{2} \frac{d v}{d x}+\frac{\rho v_{I}}{R}\left(v_{I}^{2}-v^{2}\right) \\
& +\frac{2}{R} \rho v_{I} C_{p}\left(T_{I}-T\right)+\frac{2}{R} q_{I}-\rho v g \sin \theta=0
\end{aligned}
$$

Solving for temperature gradient,

$$
\begin{aligned}
& \frac{d T}{d x}=-\frac{(1-\beta T)}{\rho C_{P}} \frac{d p}{d x}-\frac{v}{C_{P}} \frac{d v}{d x}+\frac{1}{R C_{P}} \frac{v_{I}}{v}\left(v_{I}^{2}-v^{2}\right) \\
& +\frac{2}{R} \frac{v_{I}}{v}\left(T_{I}-T\right)+\frac{2}{R \rho v C_{P}} q_{I}-\frac{1}{C_{P}} g \sin \theta
\end{aligned}
$$

Joule-Thomson coefficient is defined as

$$
\frac{\beta T-1}{\rho C_{P}}=K_{J T}
$$


Conductive heat flux from the surroundings can be estimated using the heat transfer coefficient, $U$, of the completion,

$q_{I}=U\left(T_{I}-T\right)$

And $\frac{1}{2 U / \operatorname{R} \rho v C_{P}}$ is called relaxation distance $(=A)$.

Substituting into Eq. 4.40 yields

$$
\frac{d T}{d x}=\frac{1}{C_{p}}\left(K_{J T} C_{P} \frac{d p}{d x}-v \frac{d v}{d x}+\frac{1}{R} \frac{v_{I}}{v}\left(v_{I}^{2}-v^{2}\right)-g \sin \theta\right)+\left(\frac{2}{R} \frac{v_{I}}{v}+\frac{1}{A}\right)\left(T_{I}-T\right)
$$

Now we can infer what causes temperature increase or decrease. The first term on the right-hand side of Eq. 4.43 is the Joule-Thomson effect. The second and third terms are the temperature decrease due to kinetic energy changes. The fourth term represents the work done by gravity force. The convective heat transfer is expressed by the fifth term and the conductive by sixth.

If there's no inflow to the system, the equation would be

$$
\frac{d T}{d x}=\frac{1}{C_{P}}\left(K_{J T} C_{P} \frac{d p}{d x}-v \frac{d v}{d x}-g \sin \theta\right)+\frac{1}{A}\left(T_{I}-T\right)
$$

This equation is same equation as the one derived by Shoham for non-producing wellbore temperature prediction.

\subsection{Model Development}

In the last section, we derived three equations to be solved for three unknowns. The 3 unknowns are $v, p, T$; and the 3 equations are

$$
\begin{aligned}
& \frac{d(\rho v)}{d x}=\frac{2}{R}\left(\rho v_{I}\right) \\
& \frac{d p}{d x}=-\frac{\rho v^{2} f}{R}-\alpha\left(\frac{2}{R} \rho v_{I} v+\rho v \frac{d v}{d x}\right)-\rho g \sin \theta \\
& \frac{d T}{d x}=\frac{1}{C_{P}}\left(K_{J T} C_{P} \frac{d p}{d x}-v \frac{d v}{d x}+\frac{1}{R} \frac{v_{I}}{v}\left(v_{I}^{2}-v^{2}\right)-g \sin \theta\right)+\left(\frac{2}{R} \frac{v_{I}}{v}+\frac{1}{A}\right)\left(T_{I}-T\right)
\end{aligned}
$$


For a real fluid, thermodynamic properties are dependent on pressure and temperature such as

$\rho=\rho(p, T)$

$\mu=\mu(p, T)$

$C_{p}=C_{p}(p, T)$

$\beta=\beta(p, T)$

And we have a rate dependent property which is friction factor

$$
f=f\left(N_{\mathrm{Re}}, N_{\mathrm{Re}, w}, \varepsilon\right)
$$

To solve this problem, we consider the following numerical method. Then to validate numerical model we develop an analytical solution with some simplifications.

\subsubsection{Iterative Numerical Method}

Numerically, we divide the wellbore into cells and consider $v, p, T$ as average values in the cells as shown in below figure.

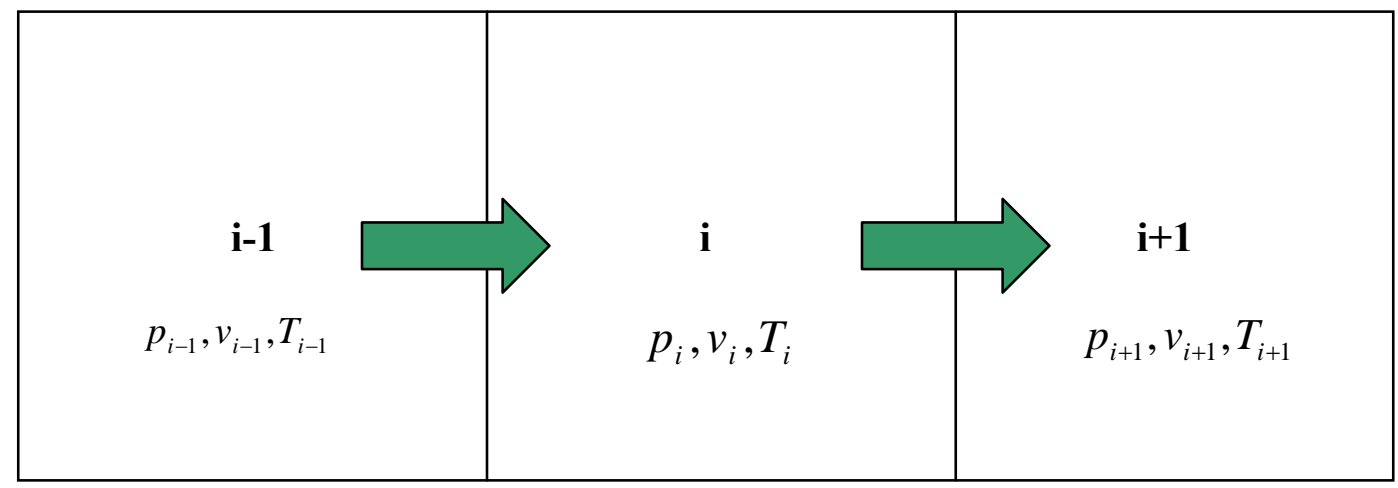

Fig. 4.4 Cell image

The procedure is as follows:

1. Start with 3 known variables $p_{i}, v_{i}, T_{i}$

2. Assume $T_{i+1}$

3. Assume $p_{i+1}$, calculate all properties with assumed temperature and pressure. 
4. Calculate $v_{i+1}$ from Mass balance using forward finite difference:

$v_{i+1}=\frac{2 \Delta x}{R} \frac{\rho_{i}}{\rho_{i+1}}\left(v_{I}\right)_{i}+\frac{\rho_{i}}{\rho_{i+1}}(v)_{i}$

For estimation of $v_{I}$, we suppose $J$ (productivity index) and $p_{R}$ (reservoir pressure) to be known, then inflow velocity is calculated as

$\left(v_{I}\right)_{i}=\frac{J_{i}\left(p_{R}-p_{i}\right)}{2 \pi R \Delta x}$

Then, the velocity is estimated by

$v_{i+1}=\frac{2 J_{i}\left(p_{R}-p_{i}\right)}{\pi R^{2}} \frac{\rho_{i}}{\rho_{i+1}}+v_{i-1} \frac{\rho_{i-1}}{\rho_{i+1}}$

5. Calculate $p_{i+1}$ from momentum balance

$$
\begin{aligned}
& \frac{p_{i+1}-p_{i}}{\Delta x}=-\frac{\rho_{i} v_{i}^{2} f_{i}}{R}-\alpha\left(\frac{2}{R} \rho_{i}\left(v_{I}\right)_{i} v_{i}+\rho_{i} v_{i} \frac{v_{i+1}-v_{1}}{\Delta x}\right)-\rho_{i} g \sin \theta_{i} \\
& p_{i+1}=\Delta x\left[-\frac{\rho_{i} v_{i}^{2} f_{i}}{R}-\alpha\left(\frac{2}{R} \rho_{i}\left(v_{I}\right)_{i} v_{i}+\rho_{i} v_{i} \frac{v_{i+1}-v_{i}}{\Delta x}\right)-\rho_{i} g \sin \theta_{i}\right]+p_{i}
\end{aligned}
$$

Friction factor and momentum correction factor are rate dependent. Therefore, if

$\left(N_{\mathrm{Re}}\right)_{i}=\frac{2 R v_{i} \rho_{i}}{\mu_{i}}<2100$ (Laminar flow), then

$\alpha=1.3333$

Friction factor with inflow is calculated by Ouyang's correlation which is

$$
f_{i}=\left(f_{o}\right)_{i}\left(1+0.0430\left(N_{\mathrm{Re}, w}\right)_{i}^{0.6142}\right)
$$

where $f_{0}$ is the friction factor without inflow, and $N_{\mathrm{Re}, w}$ is the wall Reynolds number. They can be computed as

$$
\left(N_{\mathrm{Re}, w}\right)_{i}=\frac{2 R v_{I} \rho_{i}}{\mu_{i}}
$$


$\left(f_{0}\right)_{i}=\frac{16}{\left(N_{\mathrm{Re}}\right)_{i}}$

In case if $\left(N_{\mathrm{Re}}\right)_{i}=\frac{2 R v_{i} \rho_{i}}{\mu_{i}}>2100$ (turbulent flow), then,

$\alpha=1.013$

$f_{i}=\left(f_{o}\right)_{i}\left(1-0.0153\left(N_{\mathrm{Re}, w}\right)_{i}^{0.3978}\right)$

For turbulent flow, friction factor is calculated by Chen's Equation

$\left(f_{0}\right)_{i}=\left[-4 \log \left\{\frac{\varepsilon}{3.7065}-\frac{5.0452}{\left(N_{\mathrm{Re}}\right)_{i}} \log \left[\frac{\varepsilon^{1.1098}}{2.8257}+\left(\frac{7.149}{\left(N_{\mathrm{Re}}\right)_{i}}\right)^{0.8981}\right]\right\}\right]^{-2}$

Then, compare the calculated pressure and guessed pressure. Until they match, procedure 3,4 and 5 are repeated.

6. Calculate $T_{i+1}$ from energy balance

$$
\begin{aligned}
& T_{i+1}=T_{i}+\Delta x\left[\frac{1}{\left(C_{p}\right)_{i}}\left(\left(K_{J T} C_{P}\right)_{i} \frac{p_{i+1}-p_{i}}{\Delta x}-v_{i} \frac{v_{i+1}-v_{i}}{\Delta x}+\frac{1}{R} \frac{\left(v_{I}\right)_{i}}{v_{i}}\left\{\left(v_{I}\right)_{i}^{2}-v_{i}^{2}\right\}-g \sin \theta_{i}\right)\right. \\
& \left.+\left(\frac{2}{R} \frac{\left(v_{I}\right)_{i}}{v_{i}}+\frac{1}{A_{i}}\right)\left\{\left(T_{I}\right)_{i}-T_{i}\right\}\right]
\end{aligned}
$$

Again, if temperature is not the same as the assumed one, we need to go back to the process 2 until convergence is achieved.

The whole procedure is shown in the following flow chart (Fig. 4.5).

\subsubsection{Validation of Prediction Model}

Even if we wrote numerical code and the method looked perfect mathematically, that result can never be assured unless compared with an analytical solution. Here we solve the equations for a simplified case with some assumptions in order to have a means to check the numerical model results.

To simplify the problem, we consider incompressible flow, no pipe inclination (horizontal) and inflow is uniform along the wellbore. Also, we assume thermal properties such as viscosity are constant, which is appropriate for most liquids. 


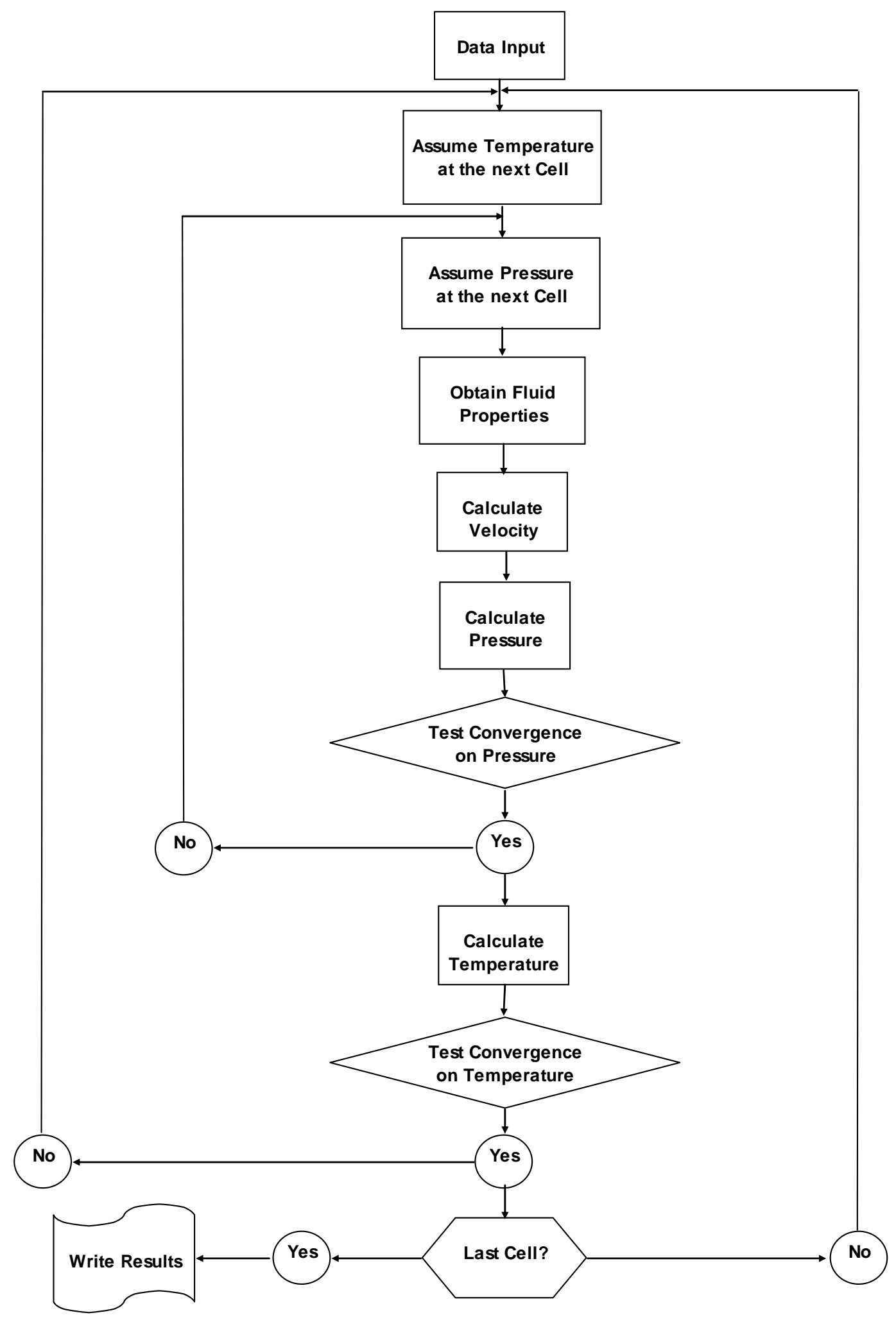

Fig. 4.5 Program flow chart 


\section{$\underline{\text { Velocity Profile }}$}

Mass balance is given as

$\frac{d(\rho v)}{d x}=\frac{2}{R}\left(\rho v_{I}\right)$

Since fluid is incompressible,

$\frac{d v}{d x}=\frac{2}{R} v_{I}$

Integrating yields

$\int d v=\int \frac{2}{R} v_{I} d x$

Applying the boundary condition, at $x=0, v=0$, the solution of velocity can be obtained.

$v=\frac{2}{R} v_{I} x$

\section{Pressure Profile}

Substituting Eqs. 4.61 and 4.62 into the momentum balance equation (Eq. 4.29), we have

$$
\frac{d p}{d x}=-\rho\left(\frac{2}{R} v_{I}\right)^{2}\left(\frac{f}{R} x^{2}+2 \alpha x\right)
$$

For an inflow rate of $50[\mathrm{bbl} / \mathrm{d} / \mathrm{ft}]$ along the producing well, the flow rate profile will be as shown in Fig. 4.6. Friction factor values corresponding to this flow profile (viscosity 1.7 [cp], density $50\left[\mathrm{lb} / \mathrm{ft}^{3}\right]$ ) are shown in Fig. 4.7. For most of the well, friction factor is constant, so we assume it constant for the entire well. Also another rate dependent property that is momentum correction $\alpha$ is almost 1 for both laminar and turbulent case. Therefore, letting $\alpha=1$ and integrating Eq. 4.63, gives

$$
\int d p=-\rho\left(\frac{2}{R} v_{I}\right)^{2} \int\left(\frac{f}{R} x^{2}+2 x\right) d x
$$

At the toe, $x=0$, pressure is $p=p_{0}$, then we obtain the solution to the pressure profile as

$$
p=p_{0}-\rho\left(\frac{2}{R} v_{I}\right)^{2}\left(\frac{f}{3 R} x^{3}+x^{2}\right)
$$




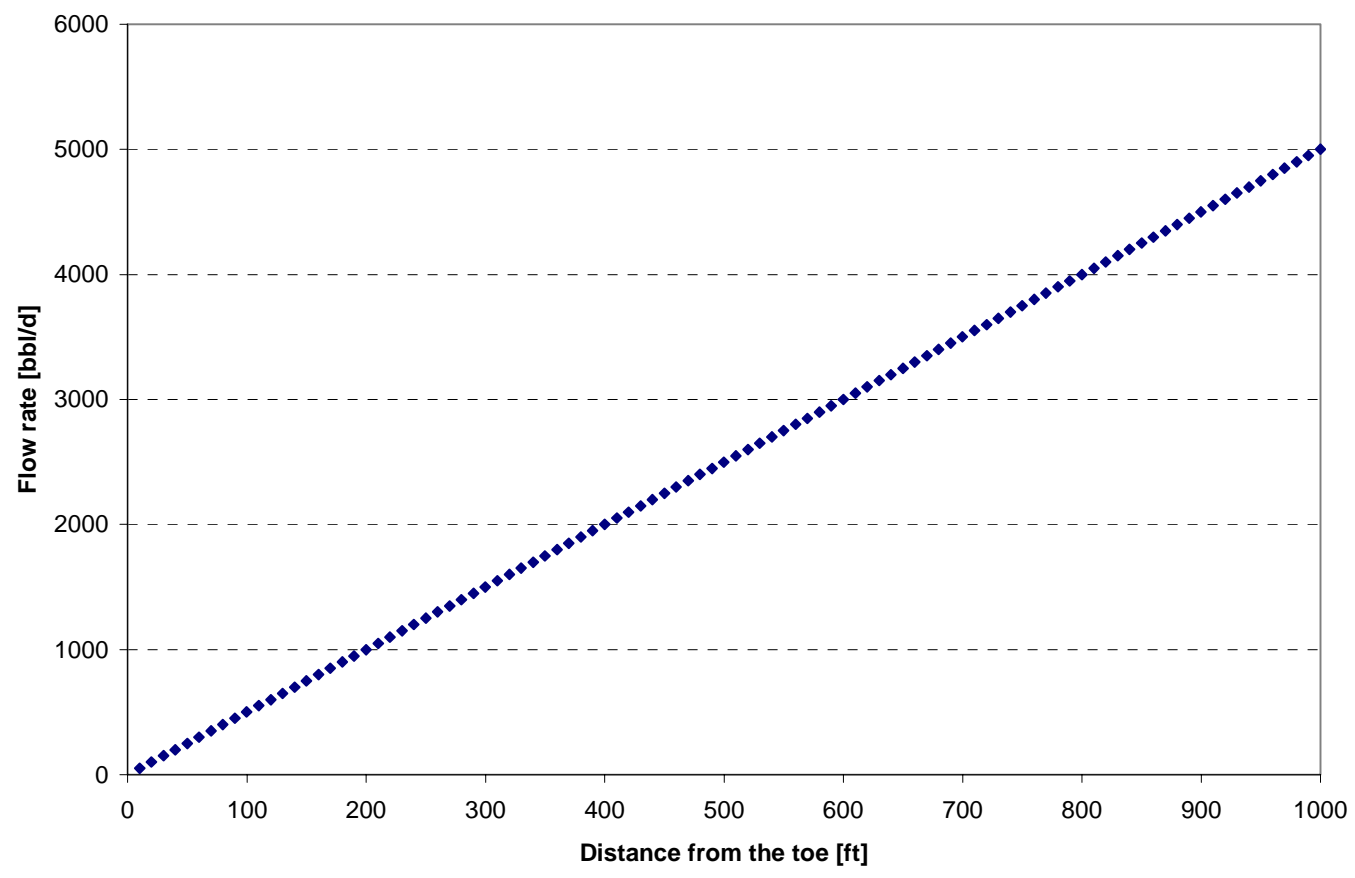

Fig. 4.6 Flow rate profile with uniform inflow

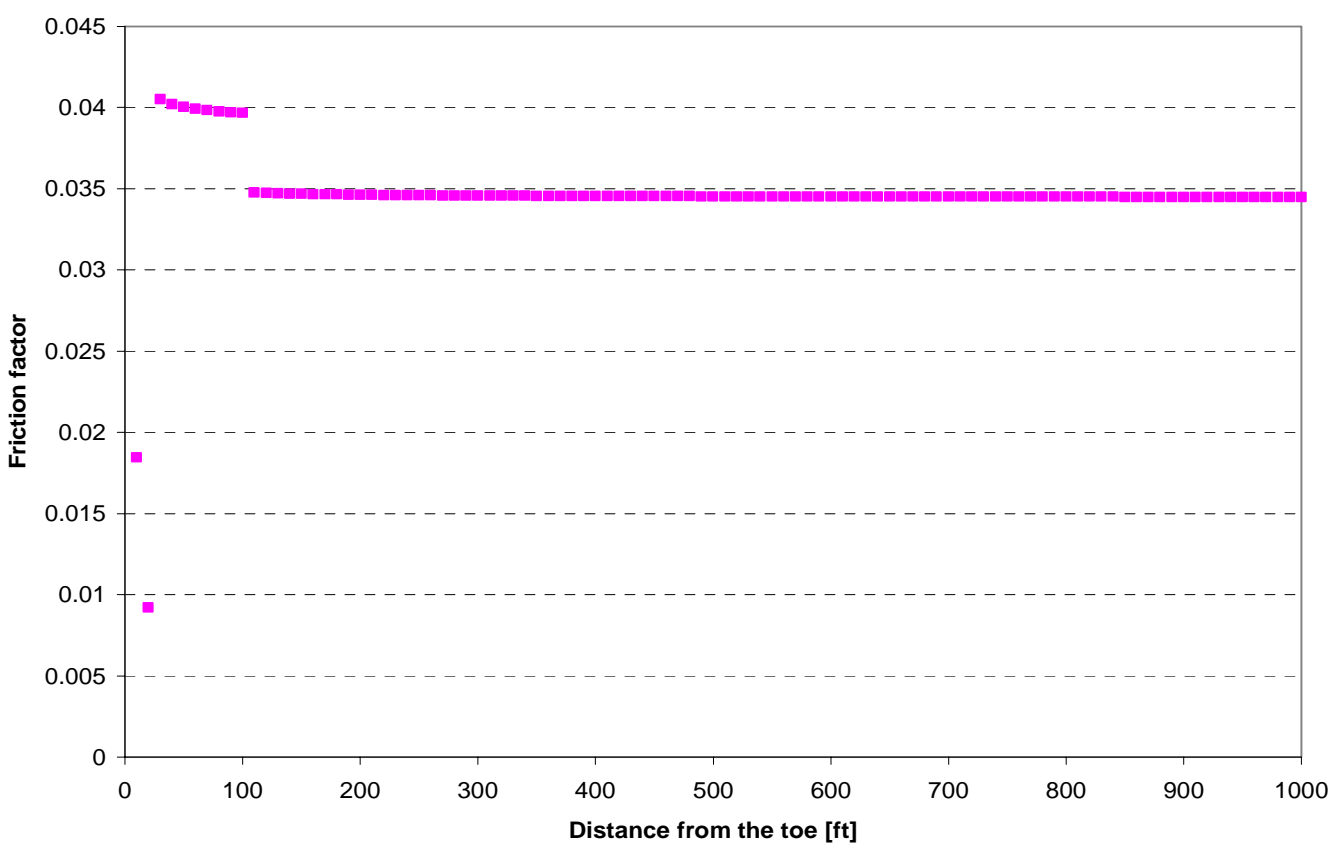

Fig. 4.7 Generated friction factor value with given flow rate profile 


\section{Temperature Profile}

With the above simplifications, the energy balance equation becomes

$$
\frac{d T}{d x}=K_{J T} \frac{d p}{d x}-\frac{v}{C_{P}} \frac{d v}{d x}+\frac{1}{R C_{P}} \frac{v_{I}}{v}\left(v_{I}^{2}-v^{2}\right)+\left(\frac{2}{R} \frac{v_{I}}{v}+\frac{2 U}{R \rho v C_{P}}\right)\left(T_{I}-T\right)
$$

We have assumed thermal properties are constant including the Joule-Thomson coefficient $\eta$. After substitution of Eqs. 4.61, 4.62 and 4.63, we obtain a $1^{\text {st }}$ order linear differential equation of the form

$$
\frac{d T}{d x}+p(x) T=q(x)
$$

Where

$$
\begin{aligned}
& p(x)=m_{1} \frac{1}{x} \\
& q(x)=m_{2} x^{2}+m_{3} x+m_{4} \frac{1}{x} \\
& m_{1}=1+\frac{U}{\rho C_{P} v_{I}} \\
& m_{2}=-\frac{4 K_{J T} f \rho v_{I}^{2}}{R^{3}} \\
& m_{3}=-\frac{8 K_{J T} \rho v_{I}^{2}}{R^{2}}-\frac{6 v_{I}^{2}}{R^{2} C_{P}} \\
& m_{4}=\frac{v_{I}^{2}}{2 C_{p}}+\left(1+\frac{U}{\rho C_{p} v_{I}}\right) T_{I}
\end{aligned}
$$

The solution is

$$
T=e^{-\int p d x}\left[\int e^{\int p d x} q d x+C\right]
$$

Integrations in the equations are

$$
e^{-\int p(x) d x}=e^{-\int\left(m_{1} \frac{1}{x}\right) d x}=C^{\prime} x^{-m_{1}}
$$




$$
\int e^{\int p(x) d x} q(x) d x=C^{\prime}\left(\frac{m_{2}}{m_{1}+3} x^{m_{1}+3}+\frac{m_{3}}{m_{1}+2} x^{m_{1}+2}+\frac{m_{4}}{m_{1}}\right)+C
$$

Putting those into the equation yields

$$
T=e^{-\int p d x}\left[\int e^{\int p d x} q d x\right]=C^{\prime}\left(\frac{m_{2}}{m_{1}+3} x^{3}+\frac{m_{3}}{m_{1}+2} x^{2}+\frac{m_{4}}{m_{1}}\right)+C x^{-m_{1}}
$$

At $x=0, T$ has a finite value, therefore $C=0$. Let $T(0)=T_{I}$, then, we have

$$
C^{\prime}=\frac{m_{1}}{m_{4}} T_{I}
$$

Finally, the solution is

$$
T=T_{I}\left(\frac{m_{1} m_{2}}{m_{4}\left(m_{1}+3\right)} x^{3}+\frac{m_{1} m_{3}}{m_{4}\left(m_{1}+2\right)} x^{2}+1\right)
$$

\section{Comparisons with Numerical Solutions}

As mentioned earlier, the validity of the prediction model has to be tested. The physical values used are shown in Table 4.1. The validation of velocity, pressure and temperature is shown by Figs. $4.8-4.10$, which compared the numerical and analytical results for the conditions of Table 4.1. The comparison is very good and within the expected error of the numerical solution.

\begin{tabular}{|c|c|}
\hline Pipe Diameter [in] & 2.295 \\
\hline Pipe Length [ft] & 1000 \\
\hline Pressure at the toe [psi] & 5800 \\
\hline Pipe Inclination [ $\left.{ }^{\circ}\right]$ & 0 \\
\hline & 6000 \\
\hline Reservoir Pressure [psi] & 180 \\
\hline Reservoir Temperature [psi] & \\
\hline Heat Capacity [btu/lb/F] & 0.52802 \\
\hline Viscosity [cp] & 1.7 \\
\hline Density [lb/ft^3] & 50 \\
\hline Thermal Conductivity [btu/hr/ft/F] & 2 \\
\hline Isothermal Expansion [1/F] & 0.000576 \\
\hline Joule-Thompson Coefficient [F $\left.{ }^{\star} \mathrm{ft}^{\wedge} 3 / \mathrm{but}\right]$ & 0.065803 \\
\hline
\end{tabular}

Table 4.1 Sample values of properties 


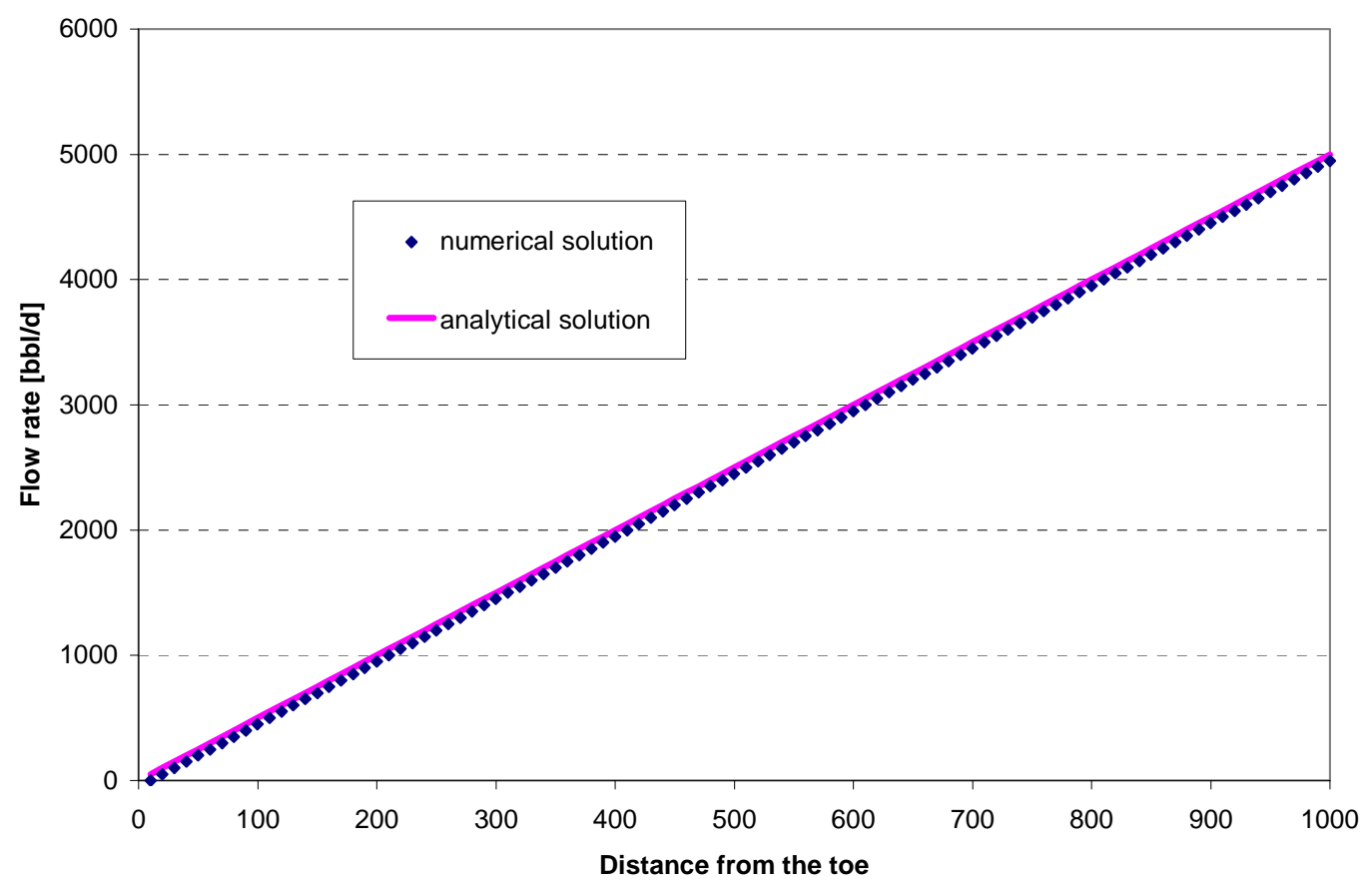

Fig. 4.8 Velocity profile comparison with prediction model

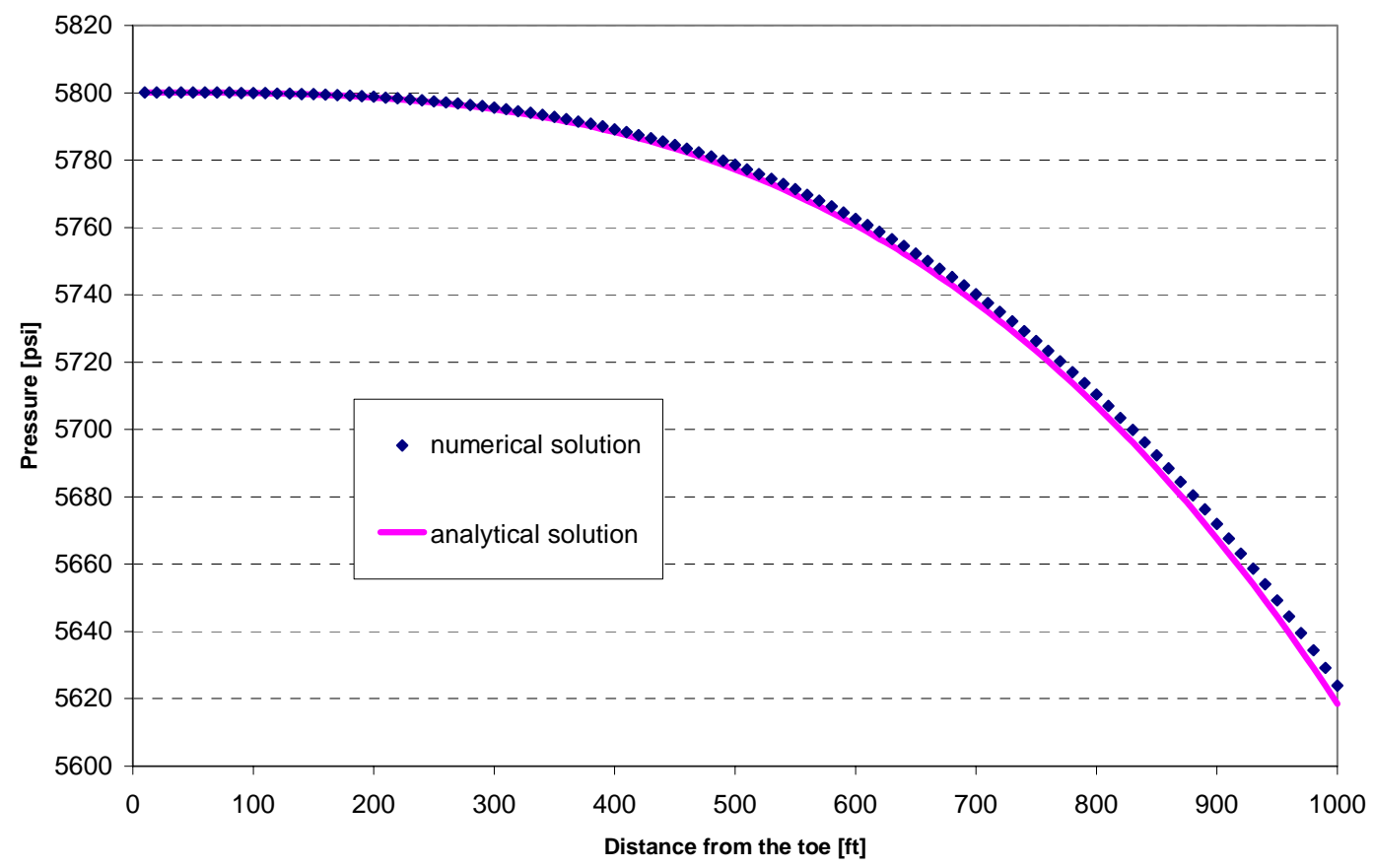

Fig. 4.9 Pressure profile comparison with prediction model 


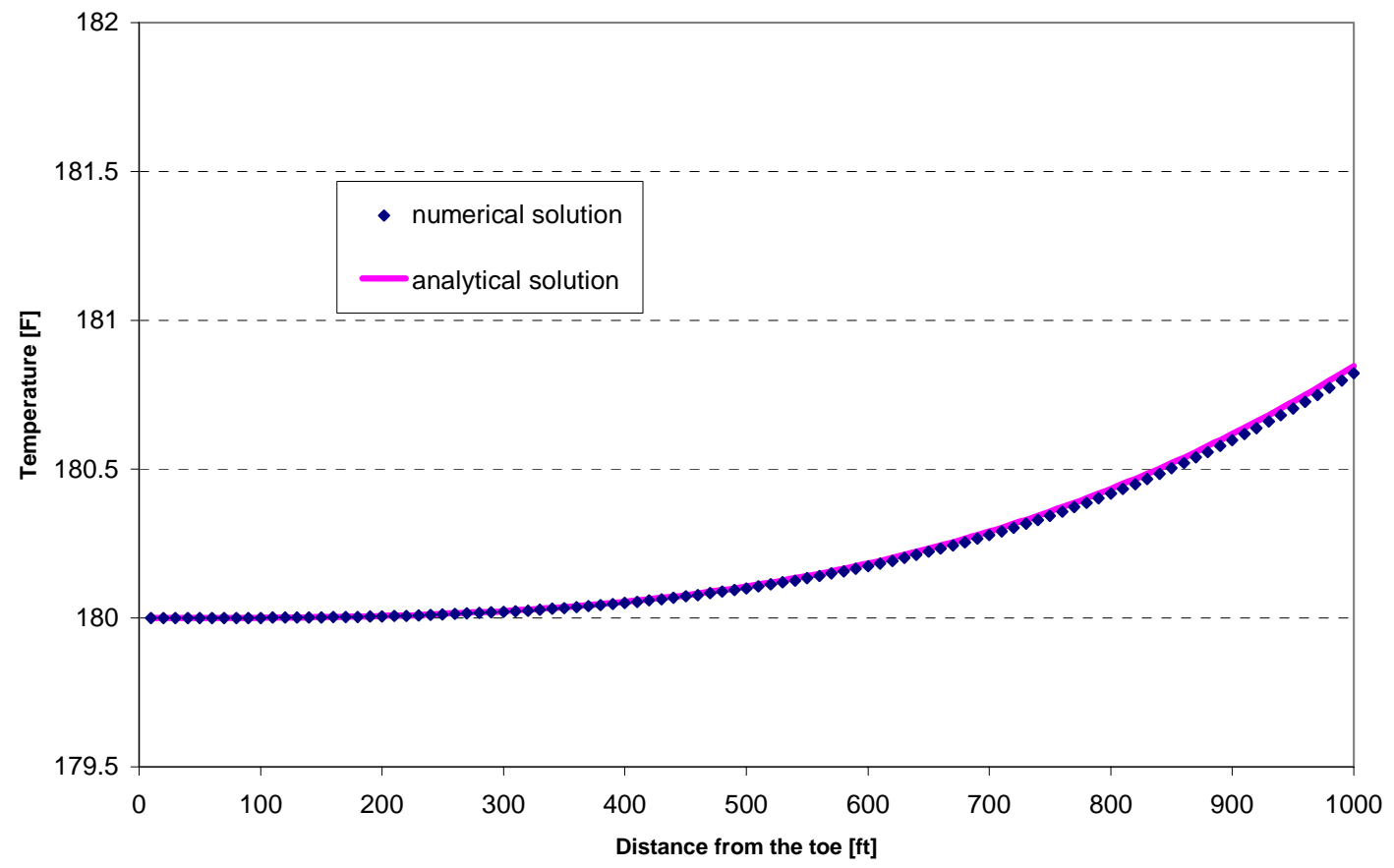

Fig. 4.10 Temperature profile comparison with prediction model

In analytical calculation, friction factors were generated after obtaining velocity profile. Then mean value was used for the solution. That averaged friction factor was adapted to numerical calculation.

Basically, in finite-difference method, the accuracy of the model will be the order of the grid size $\Delta x$. In addition, this model uses an iterative method in each step. However, from above observations, we can conclude the developed prediction model yields accurate profiles of temperature and pressure.

\subsection{Results and Discussion}

\subsubsection{Example Calculation}

We have developed the prediction model and the accuracy of the model is confirmed by an analytical solution for a simplified case. Now we can predict the profiles with realistic, more complicated cases such as compressible fluid, variable fluid properties, inflow as a function of wellbore pressure etc.

We show an example case of a compressible fluid. Inflow will be determined by productivity index and pressure difference between wellbore and reservoir. The example properties are shown in Table 4.2. The results are shown in the following figures. 


\begin{tabular}{|c|c|}
\hline Pipe Diameter [in] & 2.295 \\
\hline Pipe Length [ft] & 1000 \\
\hline Pipe Inclination [ $\left.{ }^{\circ}\right]$ & 0 \\
\hline Productivity Index [bb//d/psi] & 20 \\
\hline Pressure at the toe [psi] & 5800 \\
\hline Reservoir Pressure [psi] & \\
\hline Reservoir Temperature [psi] & 6000 \\
\hline Heat Capacity [btu//b/F] & 180 \\
\hline Viscosity [cp] & 0.52802 \\
\hline Density [lb/ft^3] & 1.7 \\
\hline Thermal Conductivity [btu/hr/ft/F] & 50 \\
\hline Isothermal Expansion [1/F] & 2 \\
\hline Isothermal Compressibility [lb/ft^3/psi] & 0.000000965 \\
\hline
\end{tabular}

Table 4.2 Properties in example calculation

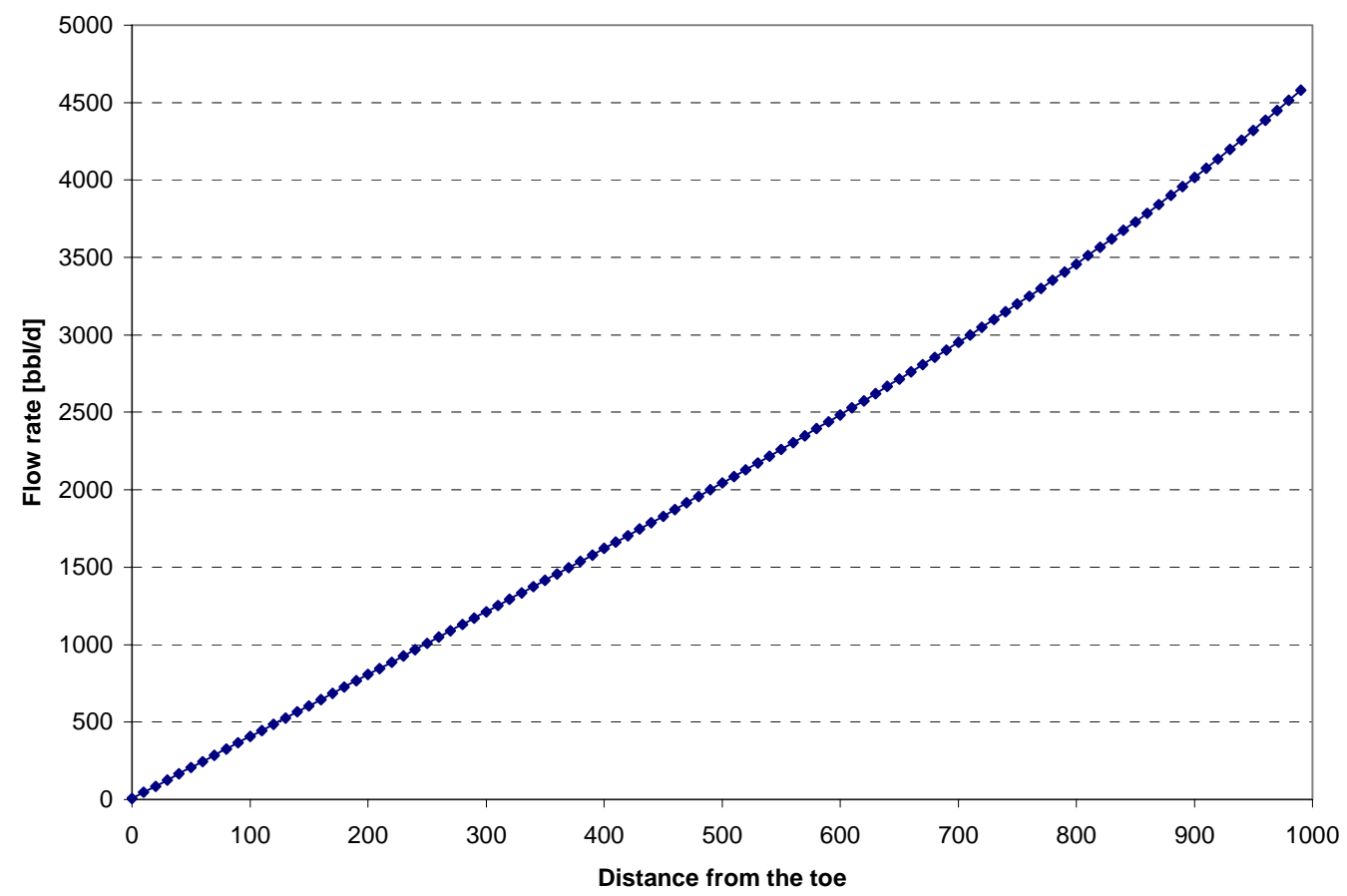

Fig. 4.11 Predicted flow rate profile 


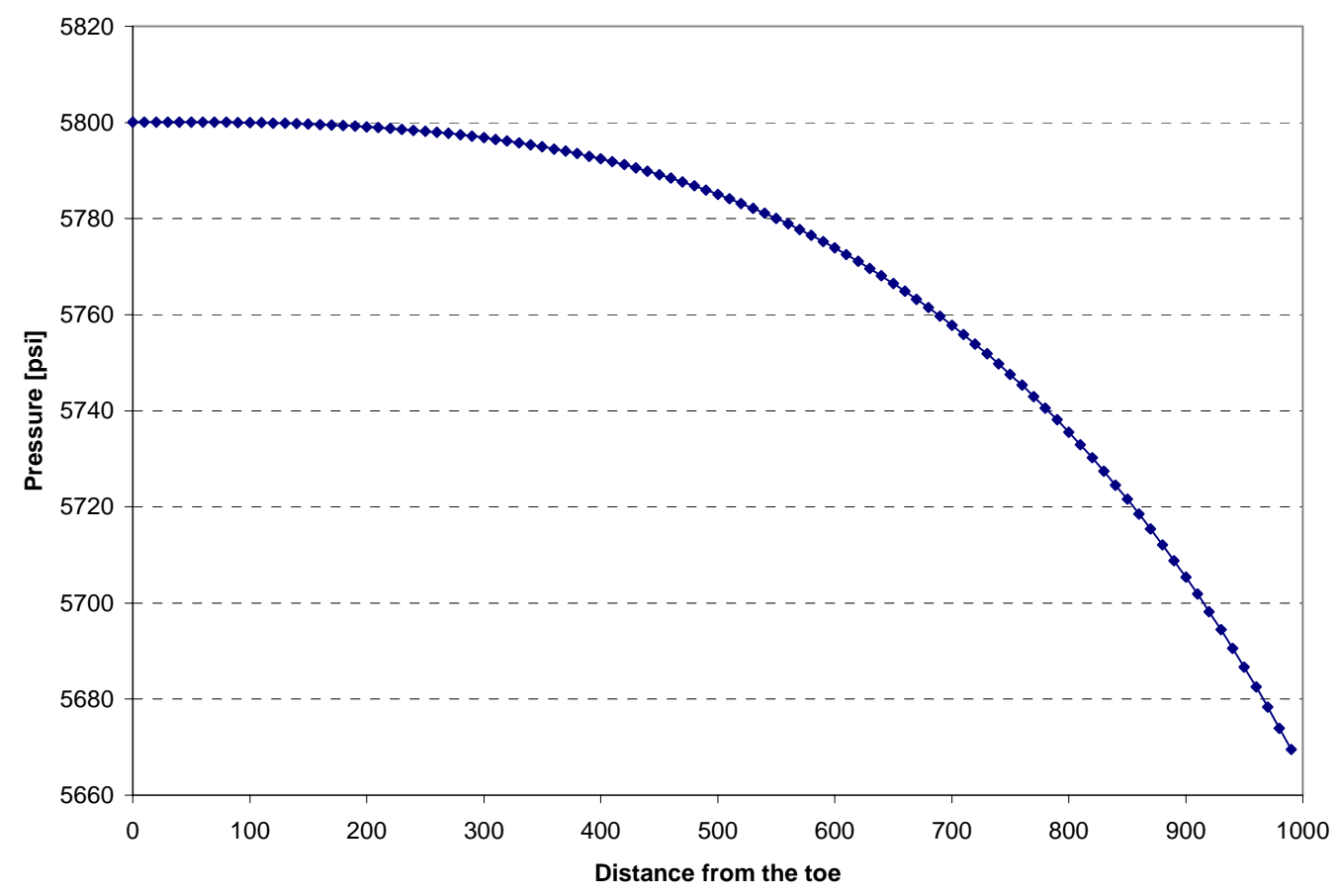

Fig. 4.12 Predicted pressure profile

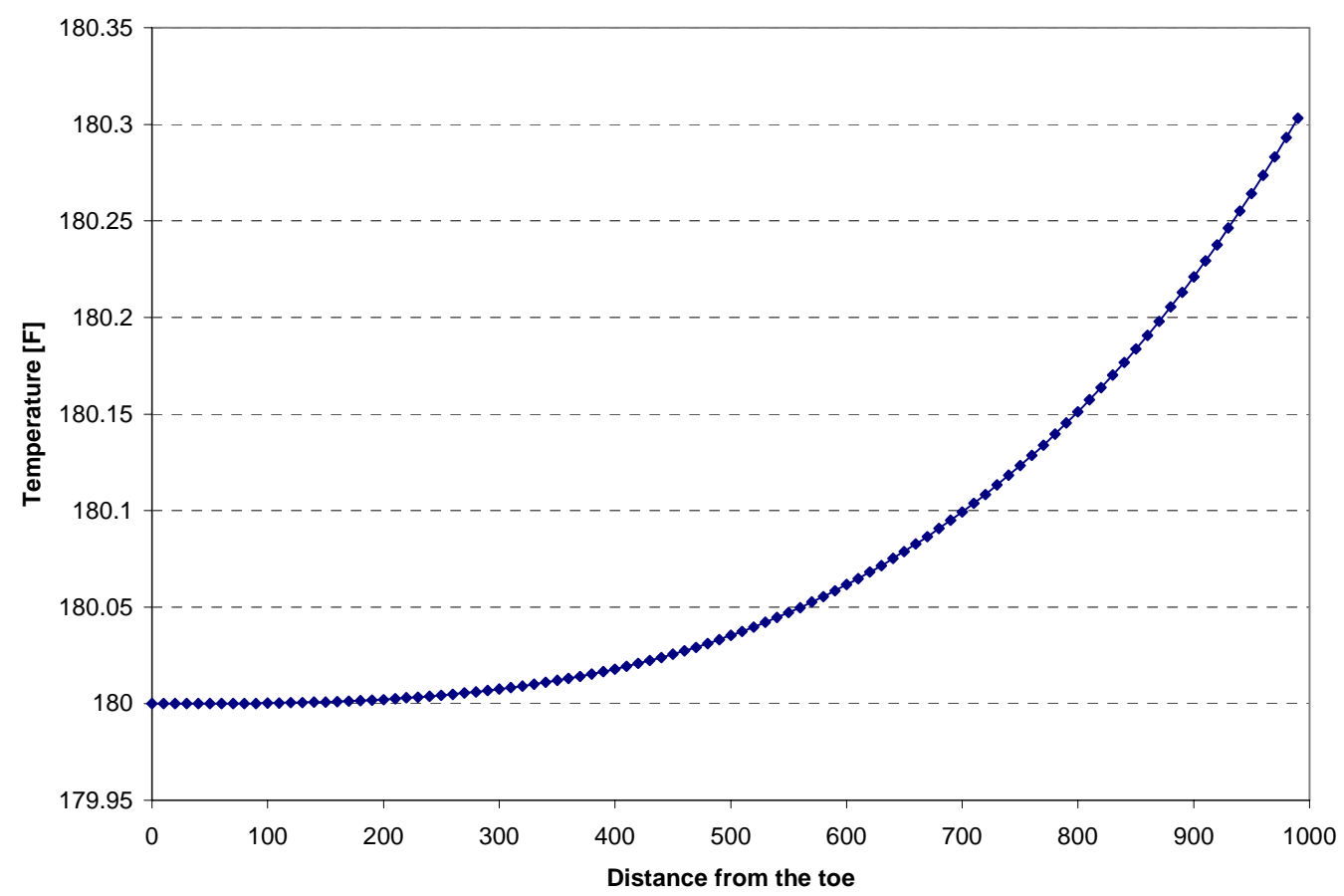

Fig. 4.13 Predicted temperature profile 
The temperature increase in this case is smaller than the incompressible case. This is caused by the smaller pressure drop. With smaller pressure drop, Joule-Thomson effect is smaller. As expected, in both cases, the temperature increases as flow rate increases.

The overall temperature increase is out about $0.3^{\circ} \mathrm{F}$. This amount is very small and would make it difficult to determine the flow rate only from the temperature increase. However we can get more information from the shape of the temperature curve and pressure profile. When we move on to the inverse problem, we should know more about sensitivity. We will through more examples, changing various data.

\subsubsection{Comparisons of Several Cases}

From the previous example, it's seen that a large pressure drop causes temperature increase due to Joule-Thomson effect. For the next case, we assume that the toe pressure is 200 psi lower than before. As a comparison, the previous data are also shown in the figures.

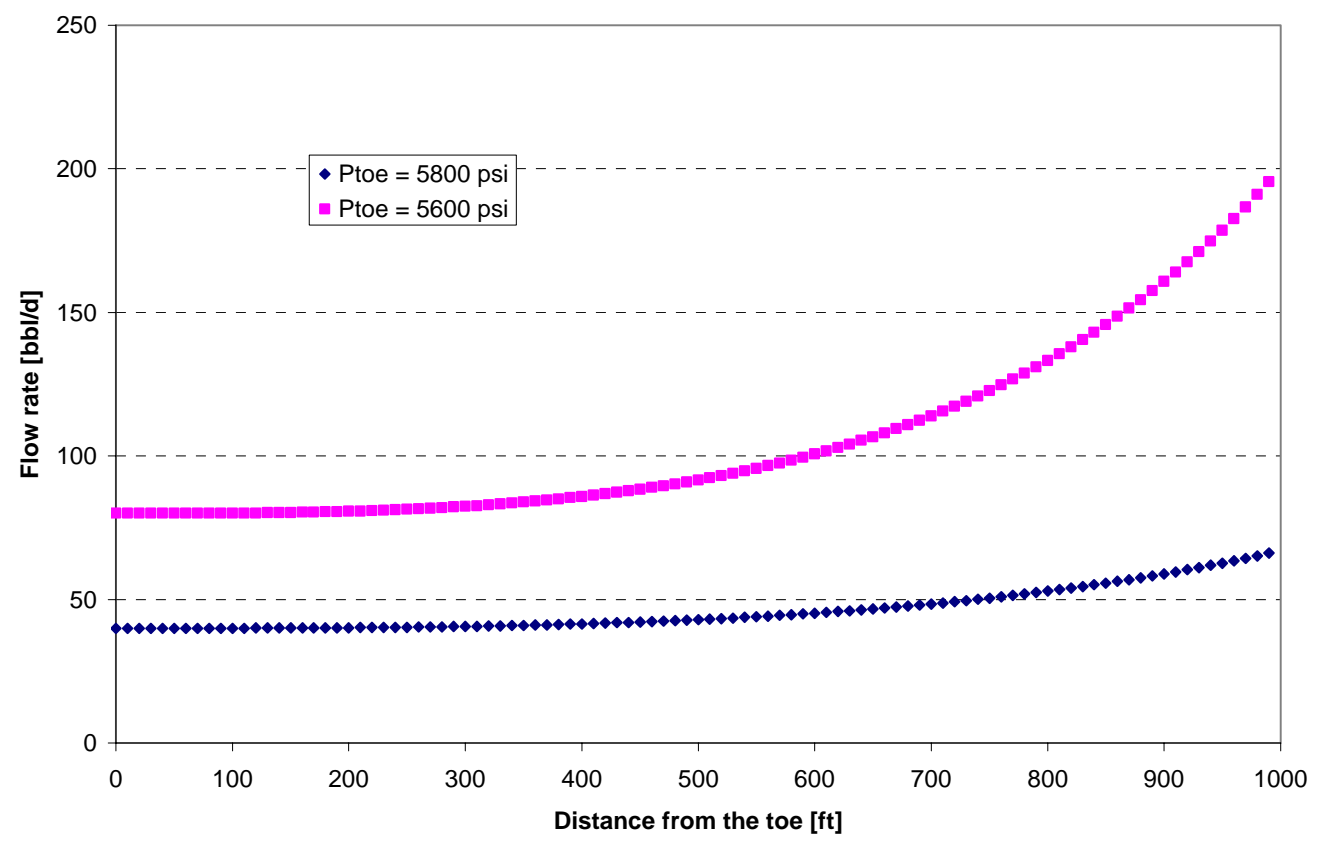

Fig. 4.14 Inflow rate profile comparison 


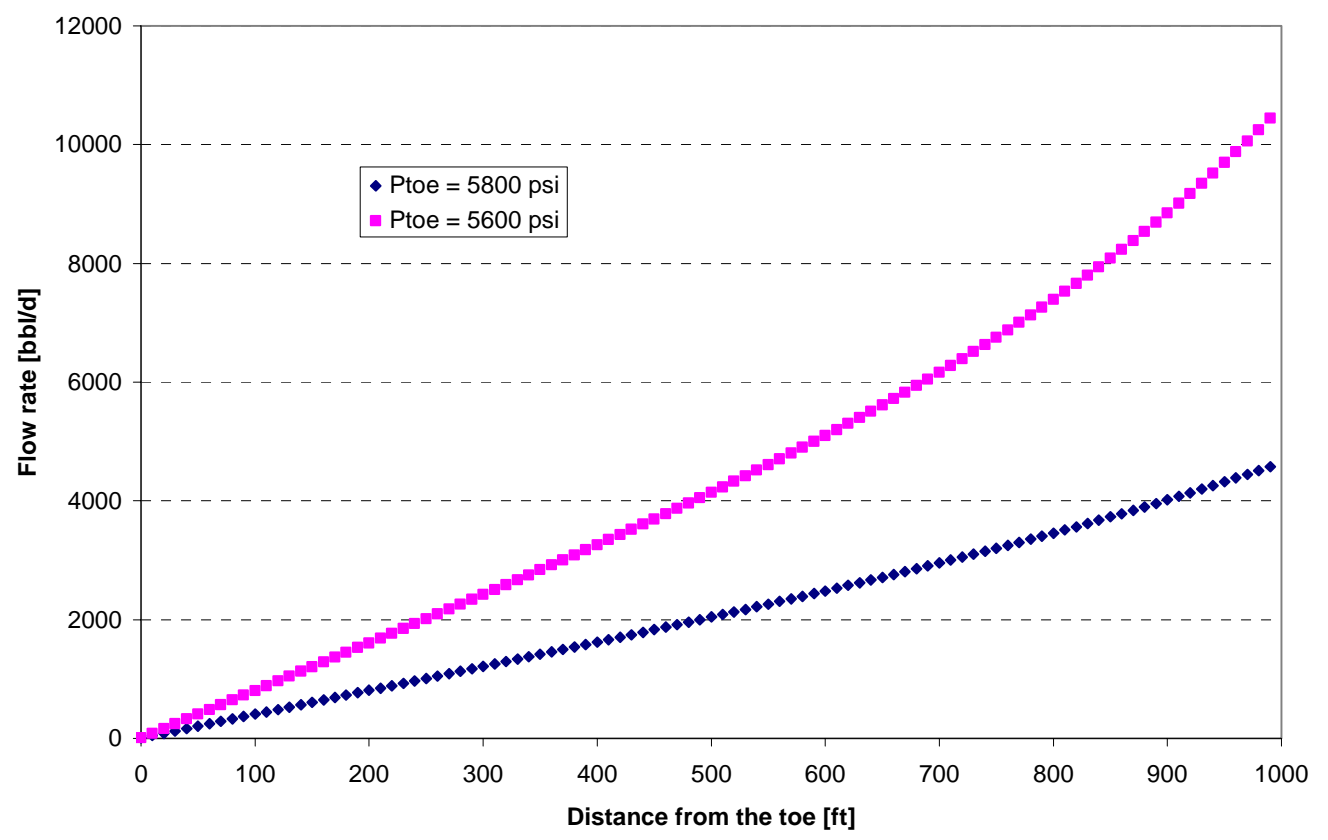

Fig. 4.15 Flow rate profile comparison

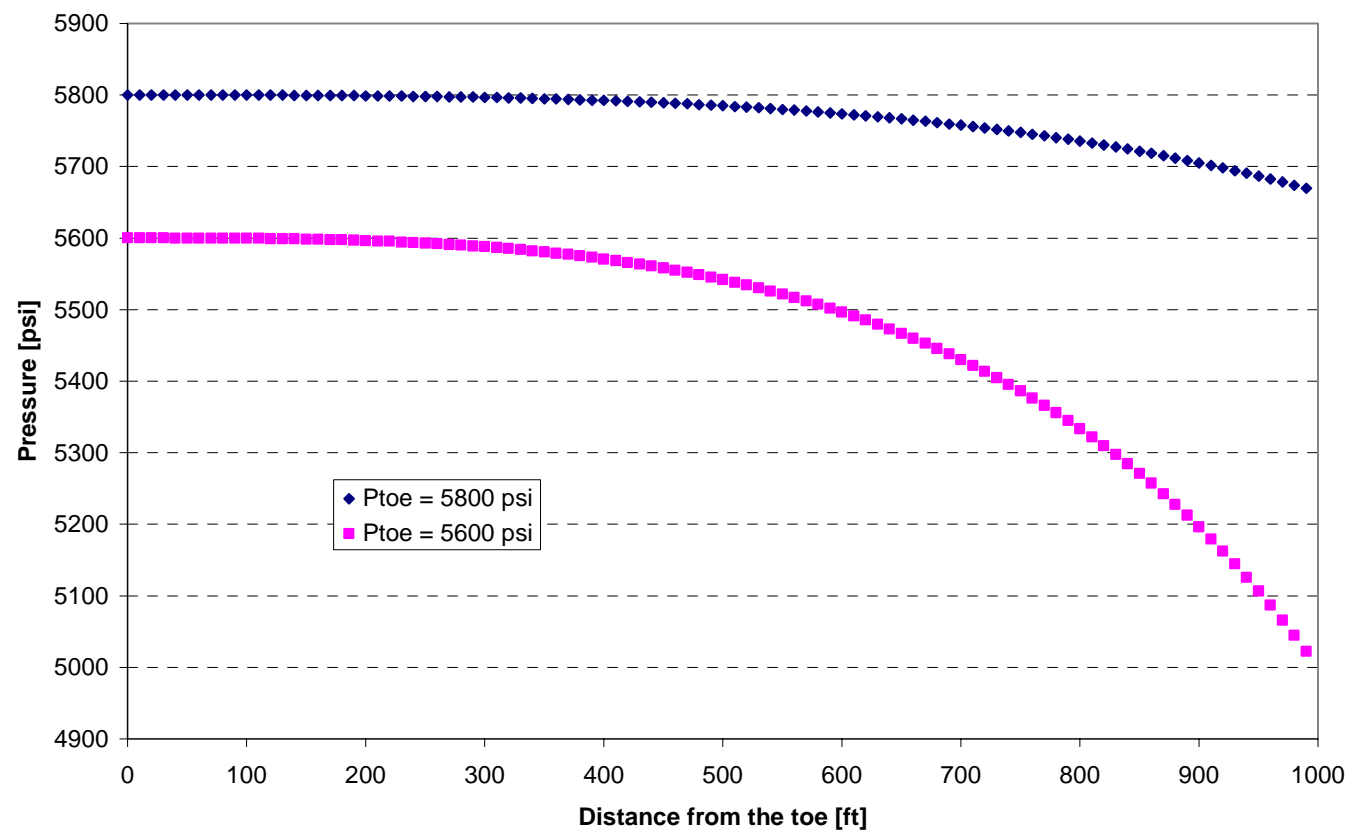

Fig. 4.16 Pressure profile comparison 


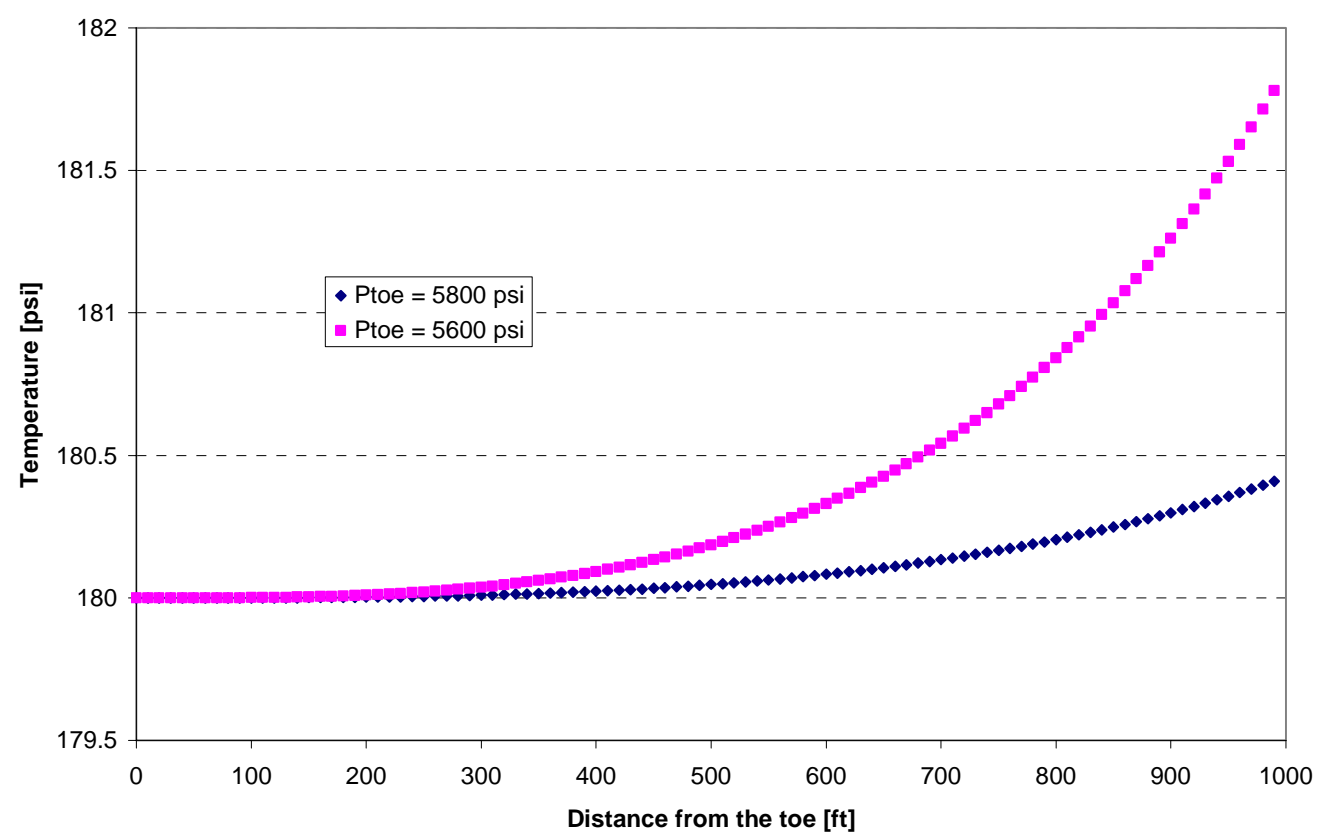

Fig. 4.17 Temperature profile comparison

This comparison shows the heel pressure difference between the two cases is 647 [psi]. And as can be seen, the temperature profile is significantly different. The temperature difference between the toe and the heel is 1.37 [F].

Those experiments are performed in uniform reservoir temperature because geothermal temperature is not considered to be different at the same depth. However, it is possible that if there is higher formation damage or lower permeability in specific regions, outside temperature distribution will be different even for the same depth. Hence, inflow from lower permeability zone is likely to have higher temperature than the other region because of frictional heating in the porous media. We next consider the situation shown in Fig. 4.18.

In this situation, formation temperature is distributed non-uniformly. In the middle there is hotter region due to the lower permeability with a $200 \mathrm{ft}$ length. We assume the productivity of lower permeability region is $50 \%$ of the other zone. Inflow, flow rate, pressure and temperature profiles are shown in followings. 
Higher

Temperature

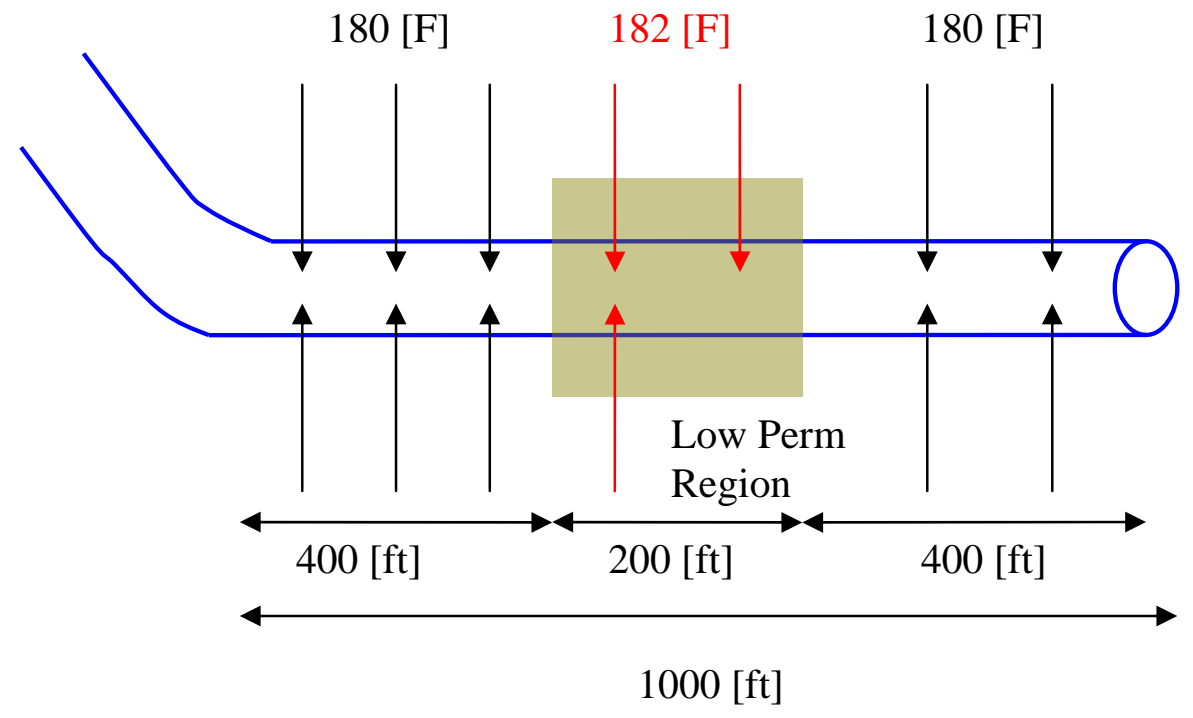

Fig. 4.18 Image of outside temperature distribution with damage zone

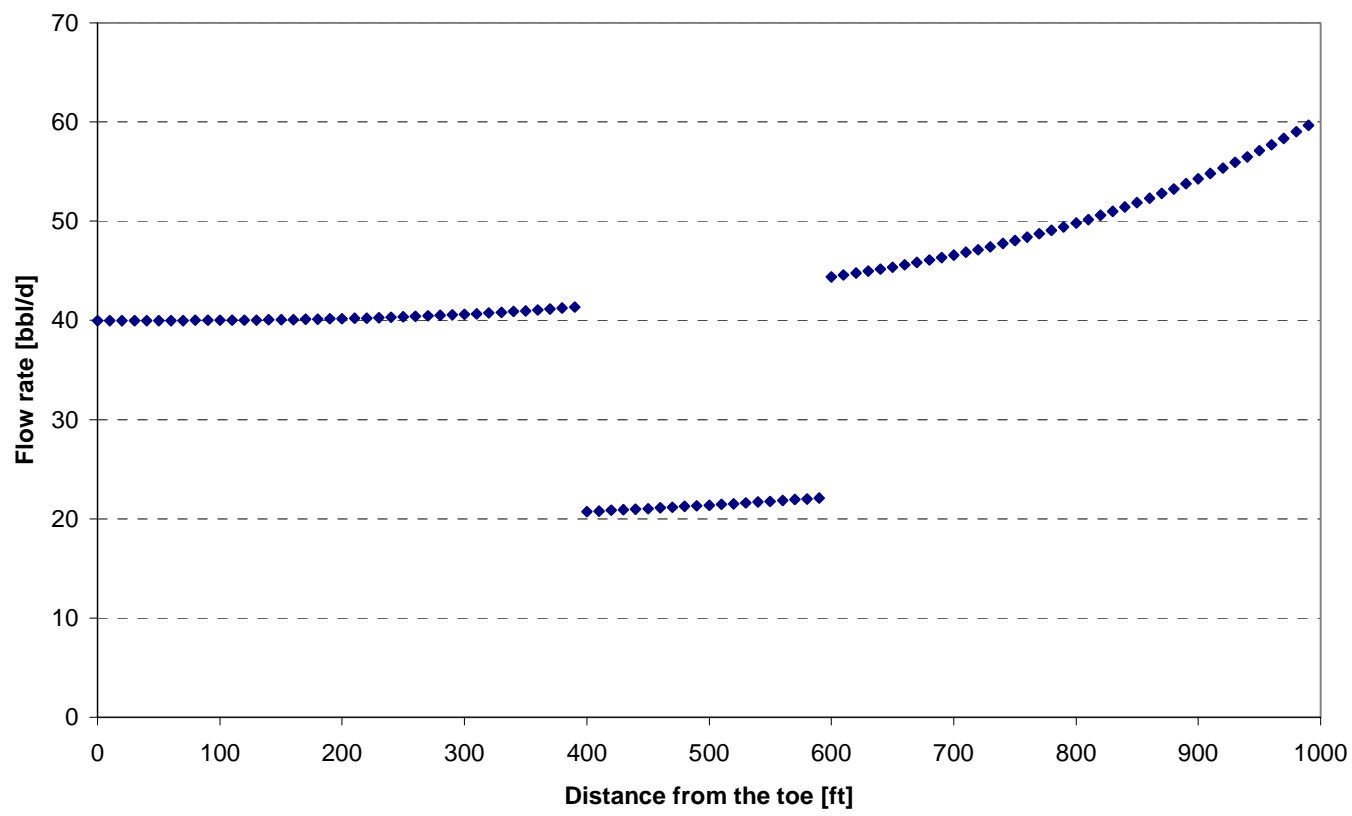

Fig. 4.19 Inflow profile with damage zone 


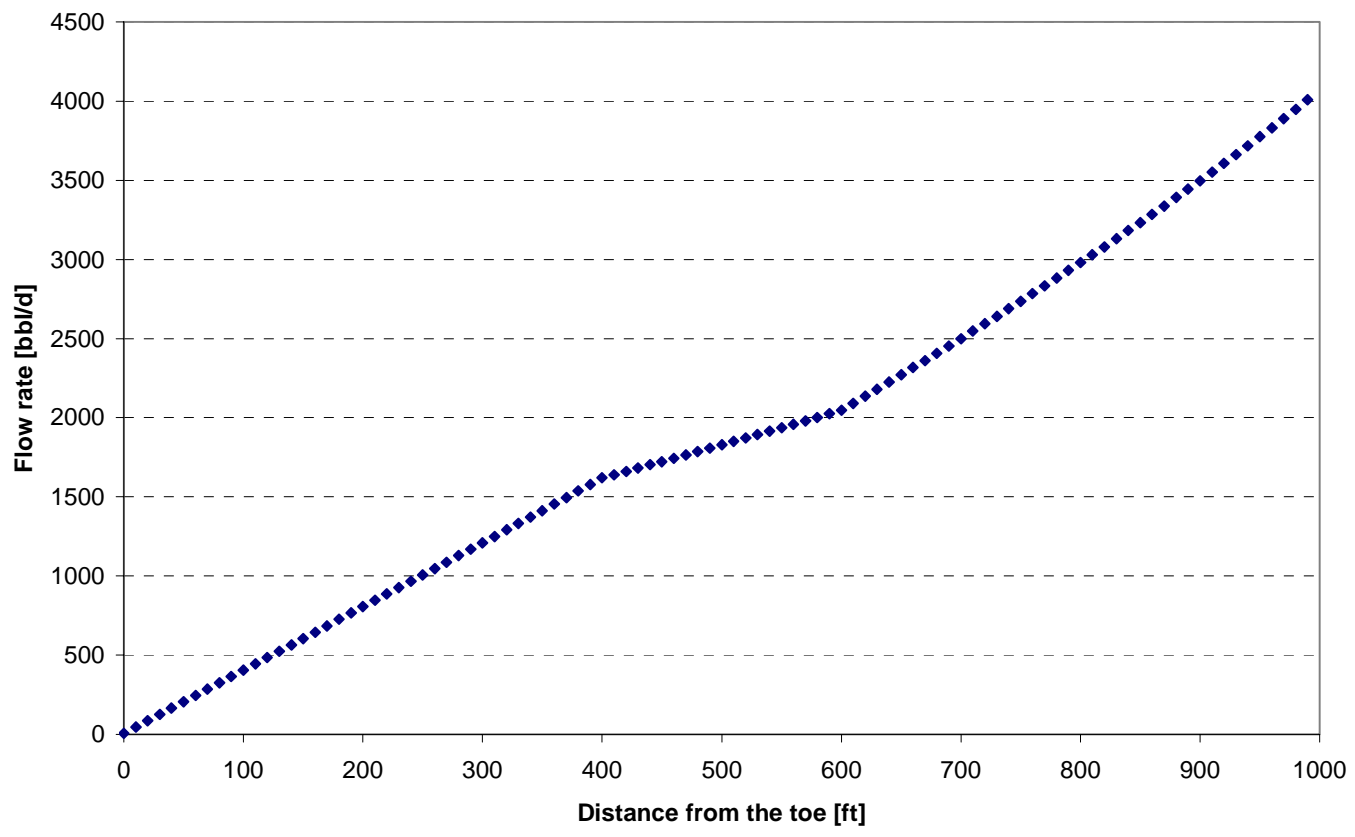

Fig. 4.20 Flow rate profile with damage zone

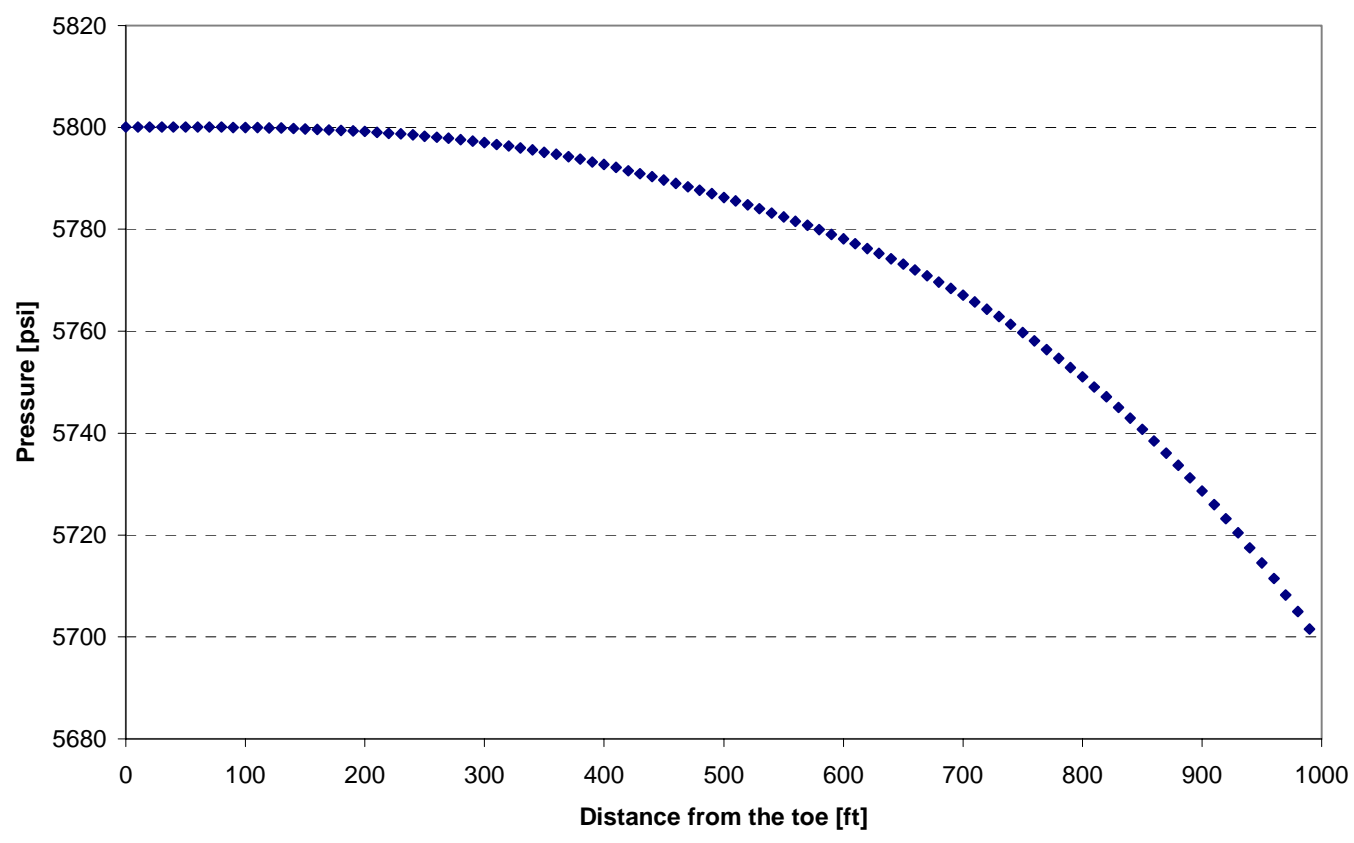

Fig. 4.21 Pressure profile with damage zone 


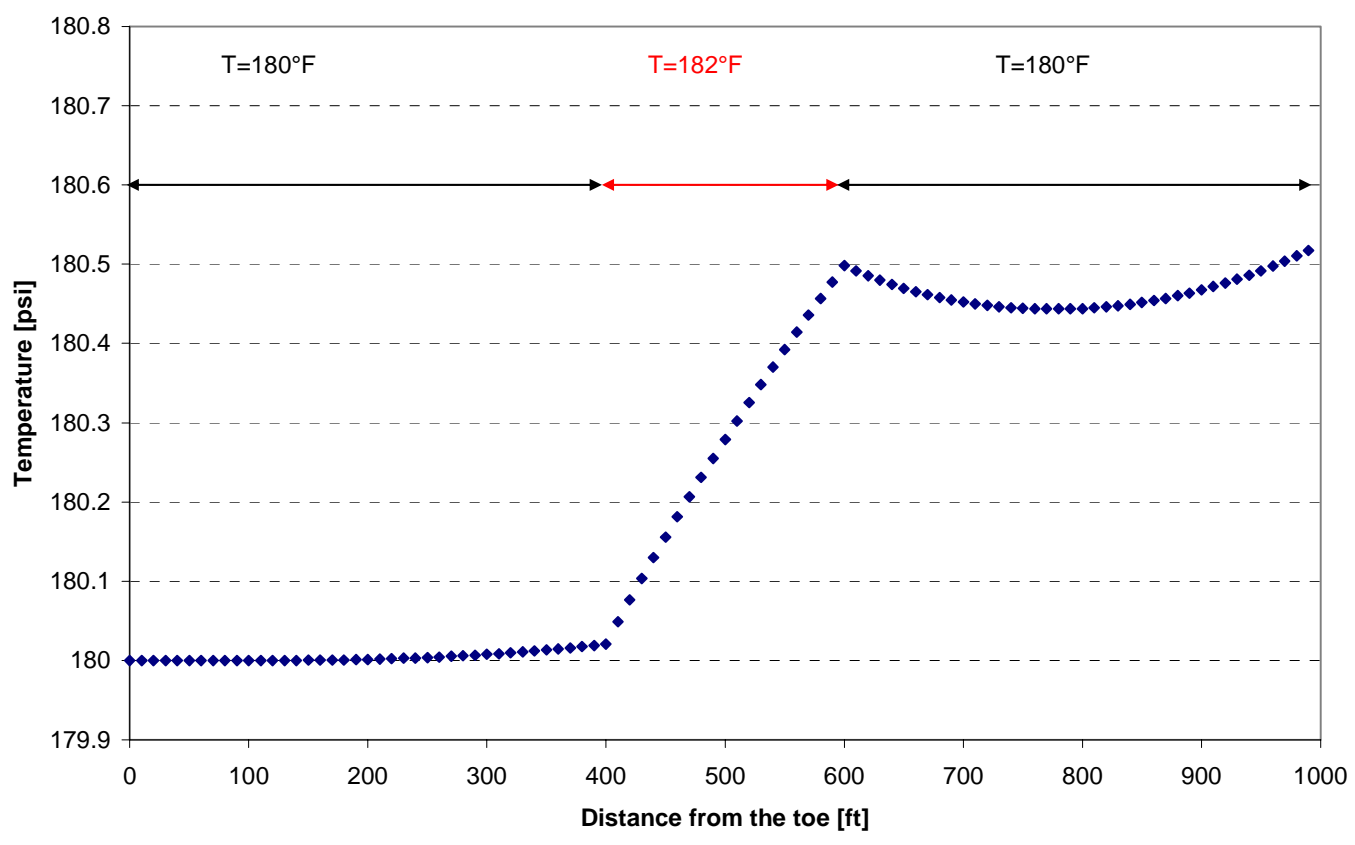

Fig 4.22 Temperature profile with hot region

In pressure profile, we barely find the discontinuity which is cased by the damage zone. This tells that 200 [ft] length low productivity zone little affects pressure drop. On the other hand, temperature is increasing with flow rate. Before hitting the hotter zone, a slight temperature increase can be observed. At the hotter region, the temperature profile becomes discontinuous and higher. An interesting thing is that the wellbore fluid requires some distance to reach the same temperature as the environment. Before obtaining same temperature, the wellbore fluid encounters normal temperature zone and gets cooler and starts heating again.

As a final example, we will see the case with production intervals, using same productivity index but there assuming two distinct production intervals. We set productivity as twice as the base case to have close amount of production. The production scheme is shown in Fig. 4.23. 


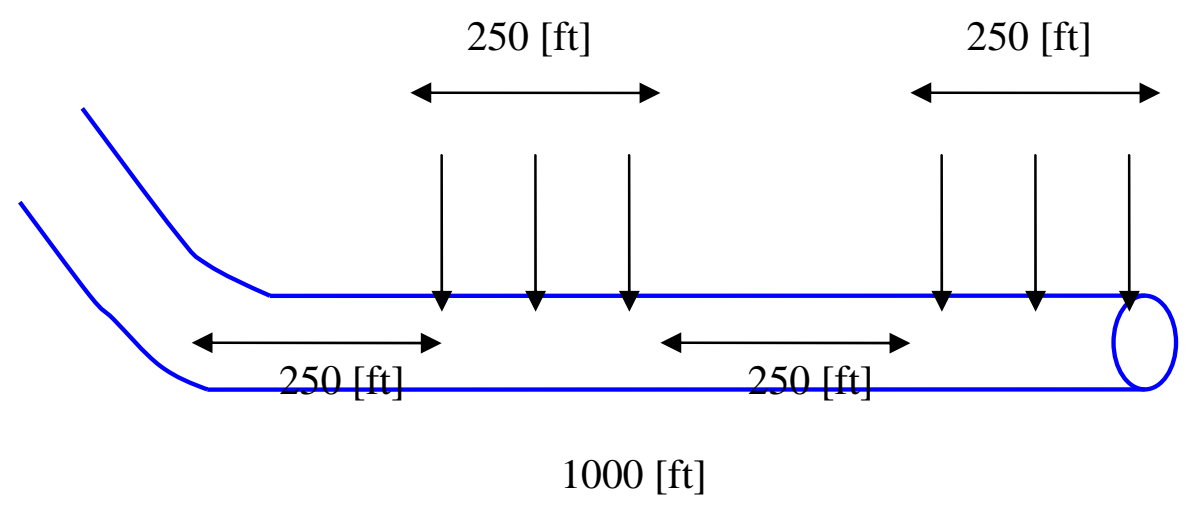

Fig. 4.23 Production scheme

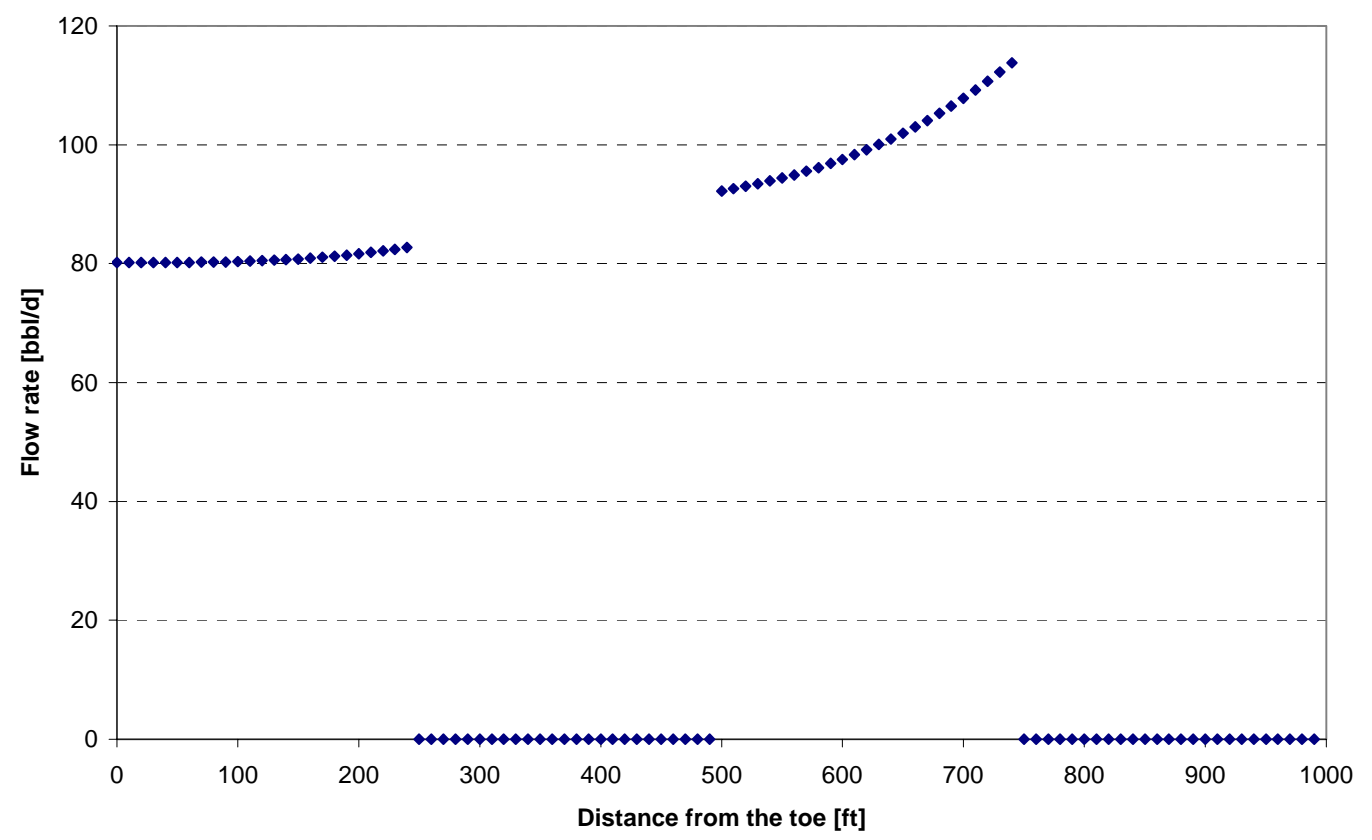

Fig. 4.24 Inflow profile with production intervals 


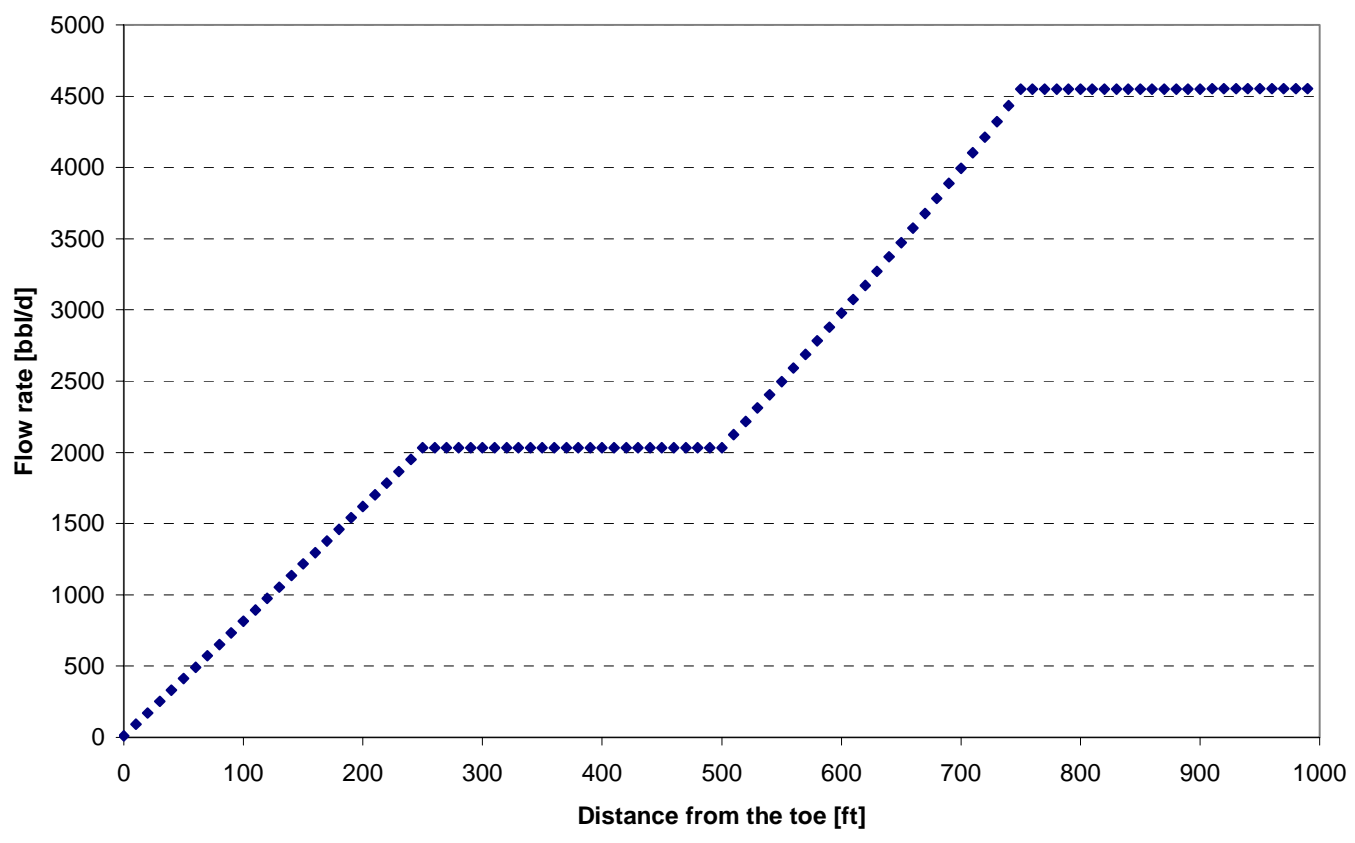

Fig. 4.25 Flow rate profile with production intervals

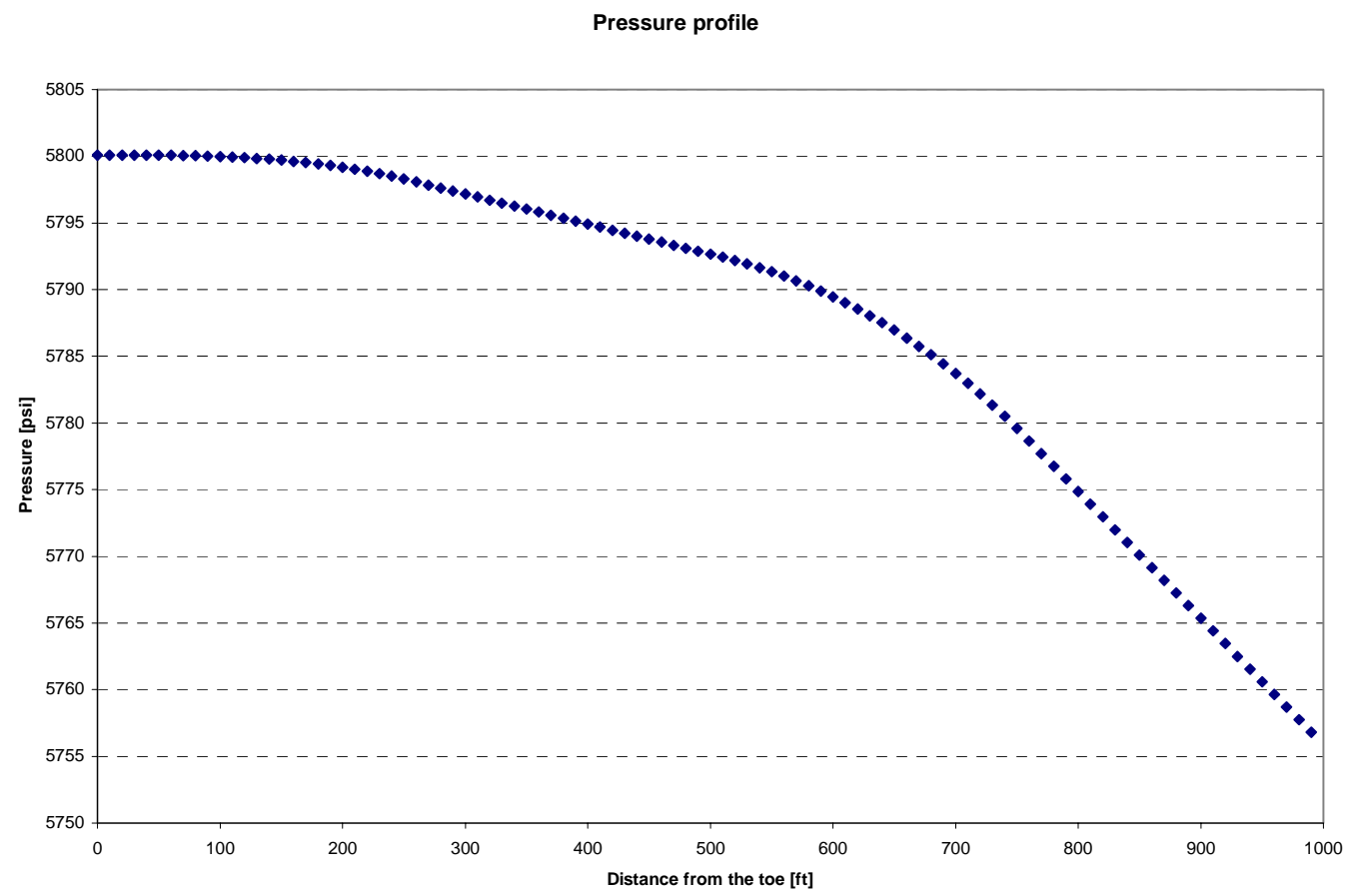

Fig. 4.26 Pressure profile with production intervals 


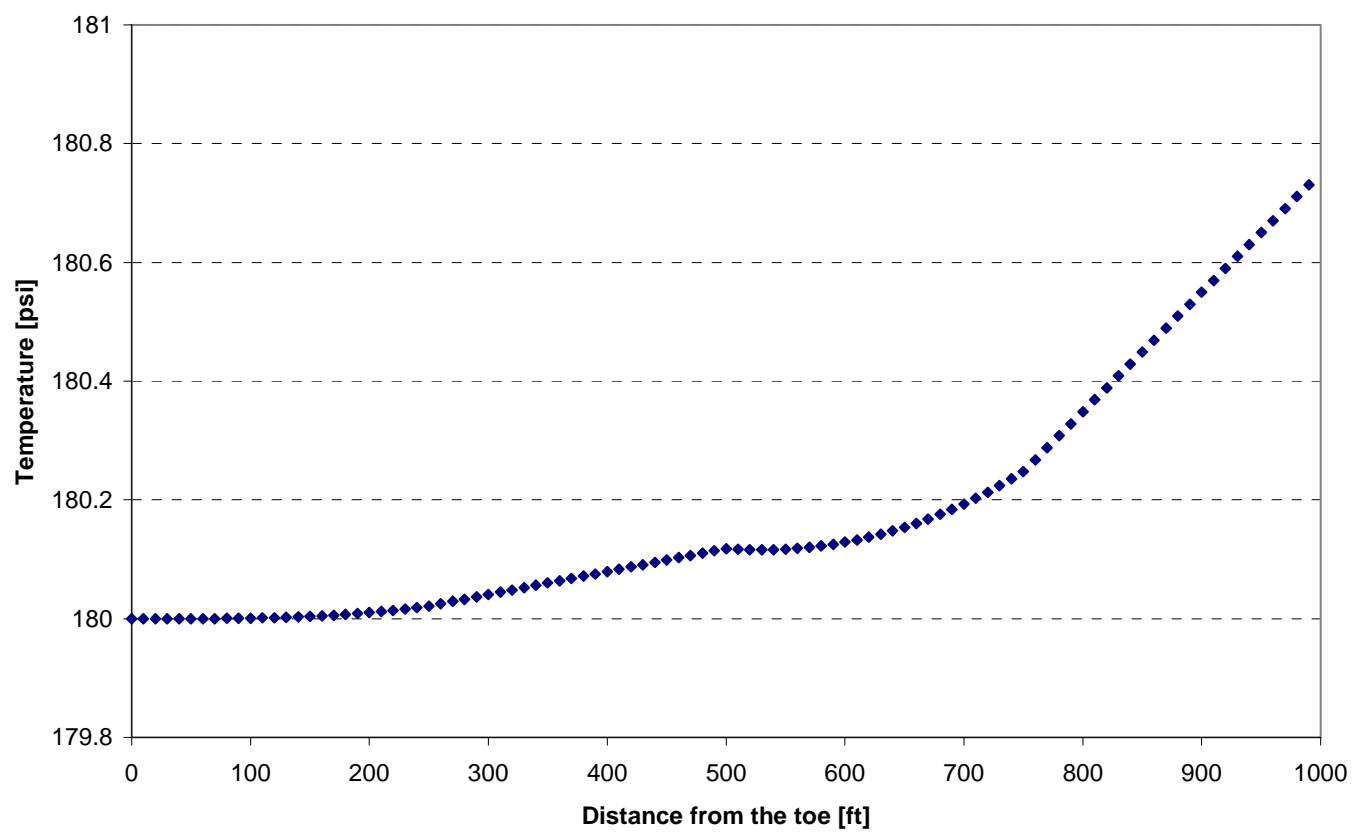

Fig. 4.27 Temperature profile with production intervals

As expected, temperature change is still small although. The important finding is that between production intervals, the profiles have discontinuities. Different intervals have different temperature curve. In the pressure profile, that discontinuity is also observed.

\section{5 Conclusion of Well Model}

The prediction model has been developed. That model yields flow rate, pressure and temperature profile along the producing wellbore with given productivity index of reservoir and boundary pressure of the wellbore. The governing equations have been derived for compressible fluid, so it can work for gas reservoir also. However, for gas, all the fluid properties will highly depend on pressure and temperature such as viscosity. The model should retain those functions in next stage. As a conclusion, the prediction model presented in this report works very well. That has been certified by comparison with analytical solution. Analytical solution is derived by simplifying the situation though, the numerical model can work more complicated situation. On the other hand, if we can regard the problem as simple one, the analytical solution may be used for it. Using analytical solution to express temperature profile will make inverse problem much easier.

As can be seen, temperature change in the wellbore is usually very small due to small geothermal change. From those results, inverse problem looks difficult only by quantity analysis. Even though, we have observed the discontinuities of temperature profile and those observations provide us valuable information other than flow rate. Also we can see discontinuities in pressure profile. It is necessary to collect sensitivity of any condition for more understanding. The relationship between fluid properties, reservoir 
condition and temperature and pressure profile have to be studied in future. Only by quantity of temperature change, inverse must be tough problem. But systematizing those parameters will make the interpretation of flow profile possible. 


\section{Model Development for Build Section}

The build section problem has two parts. First, we seek a method to calculate the temperature and pressure profile of a build section with any arbitrary well trajectory. The methods developed must be applicable to the multiphase flow conditions expected in most build sections. Second, we analyze the temperature and pressure behavior at wellbore junctions where the production streams from individual laterals are commingled.

\subsection{Temperature Profile for Single Phase Flow}

Ramey $^{23}$ made an energy balance for the fluid by assuming single-phase flow and constant angle through the fluid trajectory wellbore.

\section{Energy Balance for Wellbore Fluid}

Temperature difference between the wellbore fluid and the surrounding formation results in energy exchange. The model is derived from the total energy-balance equation over a control volume of length $\mathrm{dz}$ at a distance $\mathrm{z}$ from the wellhead shown in Fig. 5.1., where the distance coordinate, $\mathrm{z}$, is positive in the downward direction, inclined at an angle, $\theta$, to the horizontal. Assuming steady-state conditions and no work done by or to the flowing fluid. The amount of heat enters the element at $(\mathrm{z}+\mathrm{dz})$ by convection, while conduction from the formation adds $Q$ to the element. In the same way, heat leaves the element $\mathrm{z}$ by convection, adding potential and kinetic energies to the heat energy of the fluid. Thus,

$$
\frac{d H}{d z}+\frac{g \sin \theta}{g_{c} J}+\frac{v}{g_{c} J} \frac{d v}{d z}= \pm \frac{Q}{w}
$$

where the enthalpy term in Eq. 5.1 is a function of pressure and temperature and is defined as

$$
d H=C_{p} d T_{f}-K_{J T} C_{p} d p
$$




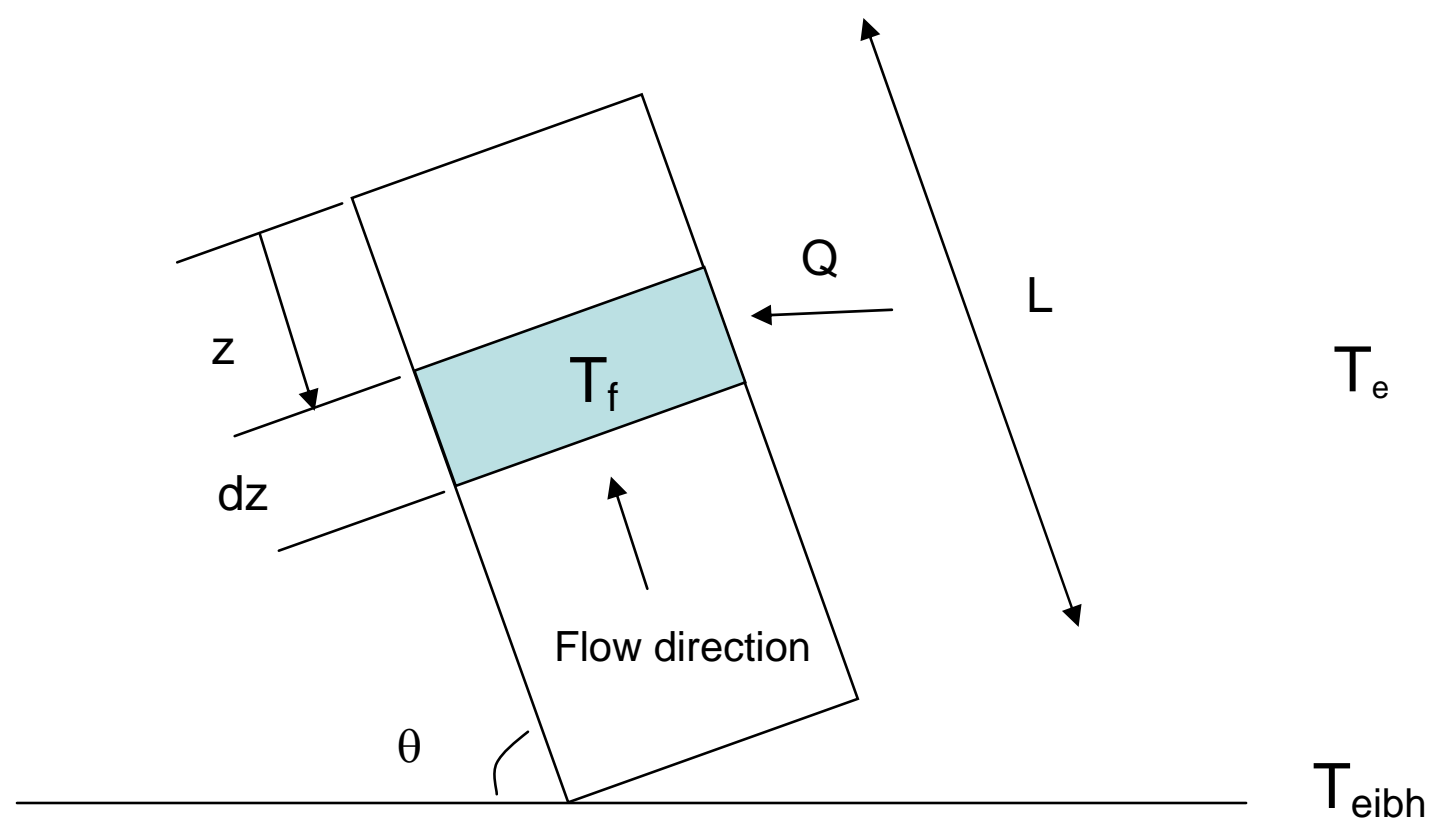

Fig. 5.1 Control volume

Substituting Eq. 5.2 into Eq. 5.1, it becomes

$$
\frac{d T_{f}}{d z}=K_{J T} \frac{d p}{d z}+\frac{1}{C_{p}}\left[ \pm \frac{Q}{w}-\frac{g \sin \theta}{J g_{c}}-\frac{v}{J g_{c}} \frac{d v}{d z}\right]
$$

The rate of heat flow at steady state condition from the wellbore fluid to the cement/earth interface is defined as,

$$
Q=-2 \pi r_{t o} U_{t o}\left(T_{f}-T_{w b}\right)
$$

assuming resistances offered by the tubing wall, tubing insulation, tubing casing-annulus, casing wall, and cement are in series, and, except for the annulus, the only energy transport mechanism is conductive heat transfer.

And the radial heat transfer from the cement/earth interface to the surrounding earth is

$$
Q \equiv-\frac{2 \pi k_{e}}{f(t)}\left(T_{w b}-T_{e i}\right)
$$

Combining Eqs. 5.4 and 5.5 yields 


$$
Q \equiv-\frac{w C_{p}}{A}\left(T_{f}-T_{e i}\right)
$$

Substituting Eq. 5.6 into Eq. 5.3, we have

$$
\frac{d T_{f}}{d z}= \pm \frac{\left(T_{f}-T_{e i}\right)}{A}-\frac{g \sin \theta}{C_{p} J g_{c}}-\frac{v}{C_{p} J g_{c}} \frac{d v}{d z}+K_{J T} \frac{d p}{d z}
$$

Assumptions made in this derivation are compressible fluid, kinetic energy change in wellbore is negligible, flowing friction is negligible, the radiation and convection coefficients are negligible and can be ignored for calculation of overall heat transfer, and because steel has a high thermal conductivity, the thermal resistance of the pipe and casing are negligible compared with the thermal resistance of the fluid in the casing-tubing annulus. Also, for a single phase liquid flow, the static head loss equals the total pressure gradient, since liquid density variation with pressure is usually very small, thus

$$
\begin{aligned}
& \frac{d p}{d z}=\rho\left(\frac{g}{g_{c}}\right) \sin \theta \\
& K_{J T} \equiv \frac{1}{C_{p}}\left[\frac{\partial H}{\partial p}\right]_{T}=\frac{V}{C_{p}}=\frac{1}{\rho C_{p}}
\end{aligned}
$$

Combining Eqs. 5.7 and 5.9, the final energy balance equation is

$$
\frac{d T_{f}}{d z}= \pm \frac{\left(T_{f}-T_{e i}\right)}{A}
$$

where we assume that the undisturbed formation temperature, $T_{e i}$, varies linearly with depth and can be expressed as

$T_{e i}=T_{e i b h}-(L-z) g_{G} \sin \theta$

Substituting Eq. 5.11 into Eq. 5.10, the energy balance equation becomes

$$
\frac{d T_{f}}{d z}=\frac{1}{A}\left\{T_{f}-\left[T_{\text {eibh }}-(L-z) g_{G} \sin \theta\right]\right\}
$$

Eq. 5.12 is a first-order linear differential equation and can be integrated. The resulting solution is

$$
T_{f}=T_{e i}+A g_{G} \sin \theta+C \exp \frac{(z-L)}{A}
$$


Or, with Eq. 5.11,

$T_{f}=T_{\text {eibh }}-(L-z) g_{G} \sin \theta+A g_{G} \sin \theta+C \exp \frac{(z-L)}{A}$

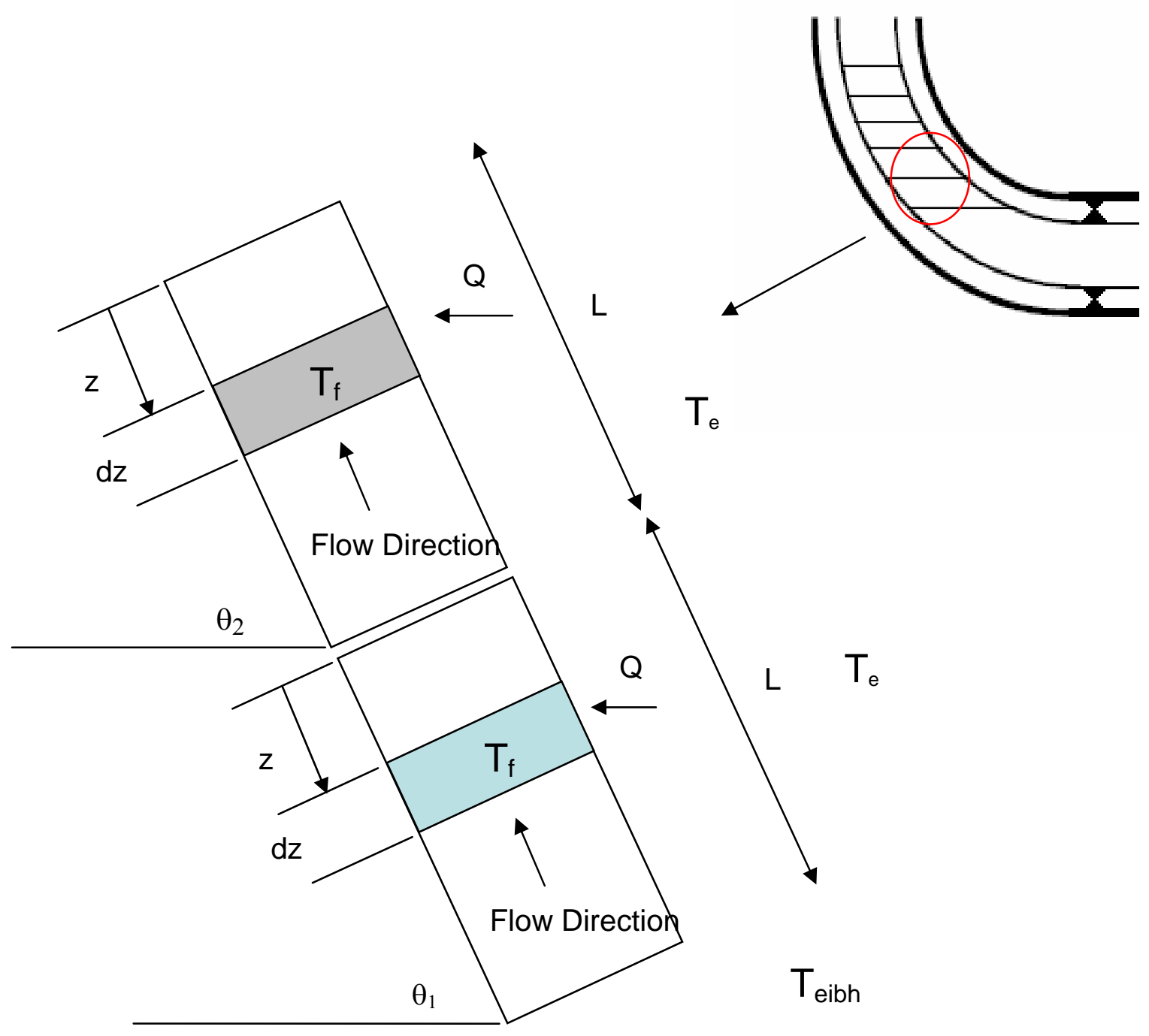

Fig. 5.2 Different boundary conditions

\section{Boundary Conditions}

Fluid entry coming from the formation, shown in Fig. 5.2, has the properties such as at the bottom hole $(\mathrm{z}=\mathrm{L})$ fluid temperature and geothermal temperature are the same at fluid entry from formation $\left(\mathrm{T}_{f}=\mathrm{T}_{\text {eibh }}\right)$ has the following integral factor,

$C=-A g_{G} \sin \theta$ 
Substitute Eq. 5.15 to 5.14, we have

$$
T_{f}=T_{e i b h}-g_{G} \sin \theta\left[(L-z)-\left(1-\exp \left(\frac{(z-L)}{A}\right)\right) A\right]
$$

For each pipe segment, as shown in Fig. 5.2., which has a constant angle which is different from the segment below it, the fluid temperature at the entrance to the segment is equal to the fluid temperature at the exit of the last segment. For this condition, the integral factor, $C$, can be defined as

$$
C=T_{f}(\text { known })-T_{e i}-A g_{G} \sin \theta
$$

Then,

$T_{f}=T_{e i}+A g_{G} \sin \theta+\left[T_{f}(\right.$ known $\left.)-T_{e i}-A g_{G} \sin \theta\right] \exp \frac{(z-L)}{A}$

\section{Overall Heat Transfer Coefficient for Casing Flow}

It is assumed that the radiation and convection coefficients are negligible and can be ignored for calculation of overall heat transfer. Because in general steels have higher thermal conductivities compared with cement, the thermal resistance of the casing is negligible compared with the thermal resistance of the cement. The overall heat transfer coefficient, $U$, for the flow in the casing is

$$
U=\frac{12}{r_{c i}}\left[\frac{\ln \left(r_{w b} / r_{c o}\right)}{k_{c e m}}\right]^{-1}
$$

and the relaxation distance parameter, $A$, is

$$
A=\left(\left(\frac{2 \pi}{w C_{p}}\right)\left[\frac{r_{c i} U k_{e}}{k_{e}+r_{c i} U f(t) / 12}\right] \frac{1}{86400 \times 12}\right)^{-1}
$$

If $f(t) r_{c i} U$ is large compared to the thermal conductivity, $k_{e}$, then $A$ simplifies to

$$
A=\frac{w C_{p} f(t)}{2 \pi k_{e}}
$$

For long times, $f(t)$ can be approximated

$$
f(t)=-0.272\left(r_{w b}\right)+3.53
$$




\section{$\underline{\text { Angle Changes along Build Section }}$}

To derive the temperature equation for the curved build section, we considered the trajectory follows the path of a curve (a quarter of a circle), and divided the true vertical depth of build section in equal segments. Knowing the distance from vertical and horizontal section, which would be the radius of a circle as shown in Fig. 5.3.The angles for different segments were calculated as follows:

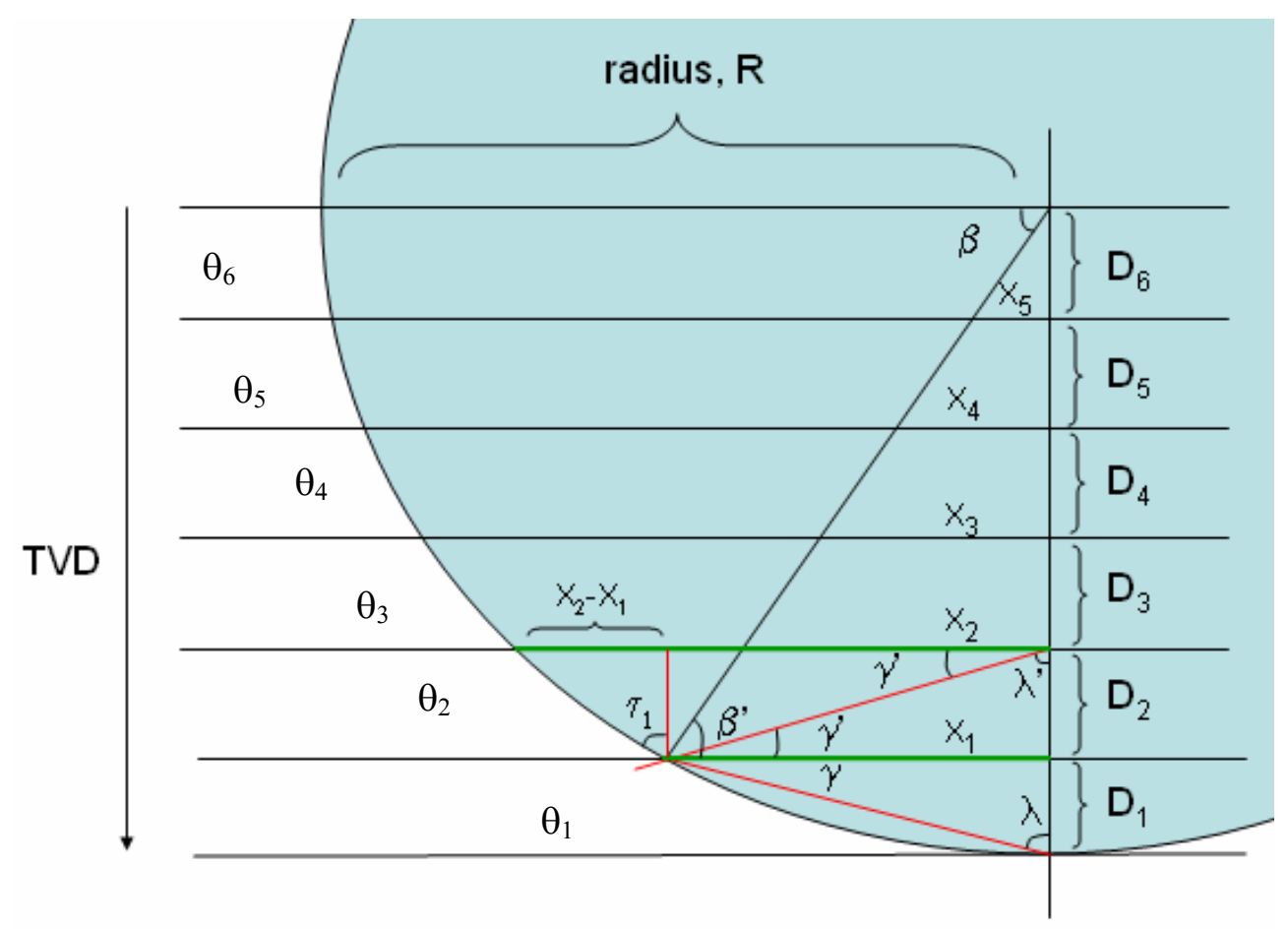

Fig. 5.3 Variable angle calculation

$\theta_{1} \neq \theta_{2} \neq \theta_{3} \neq \theta_{4} \neq \theta_{5} \neq \theta_{6}$

$D_{1}=D_{2}=D_{3}=D_{4}=D_{5}=D_{6}$

$\beta=\arcsin \left(\frac{D_{5}+D_{4}+D_{3}+D_{2}+D_{1}}{R}\right)$

and

$X_{1}=\cos (\beta) \times R$

For the first segment, 
$\theta_{1}=\arctan \left(\frac{D_{1}}{X_{1}}\right)$

For other segments,

$\theta_{n}=180-\tau_{n}$

$\tau_{n}=\arctan \left(\frac{X_{n}-X_{n-1}}{D_{n}}\right)$

\subsection{Temperature at Junctions}

The McKinley's mixing method ${ }^{15}$ can be applied to a junction with two streams mixed at the junction resulting in an enthalpy balance given by

$w_{1} C_{p 1}\left(T_{m}-T_{1}\right)+w_{2} C_{p 2}\left(T_{m}-T_{2}\right)=0$

where temperature of mixture can be expressed as

$T_{m}=\frac{w_{1} C_{p 1} T_{1}+w_{2} C_{p 2} T_{2}}{w_{1} C_{p 1}+w_{2} C_{p 2}}$

The heat capacity of a mixture, $C_{p m}$, is defined as

$C_{p m}=\left(\frac{w_{1}}{w_{1}+w_{2}}\right) C_{p 1}+\left(\frac{w_{2}}{w_{1}+w_{2}}\right) C_{p 2}$ 


\subsection{Results and Discussion}

\subsubsection{Temperature Profile along the build section}

Temperature profiles considering constant angle $\left(90^{\circ}, 45^{\circ}, 25^{\circ}, 10.5^{\circ}\right)$ and variable angle along the build section were calculated using the data given in Table 5.1. From the results shown in Fig. 5.4, it can be seen that taking a constant angle of $45^{\circ}$ through the build section would result in underestimating the temperature while taking a constant angle of $90^{\circ}$ could result in overestimating the temperature at the end of the build section compared with the temperature profile using variable angles.

\subsubsection{Temperature Profile along the Build Section and Mixed Zone}

In the next cases, temperature profiles from two laterals that are joined at a junction were calculated using the enthalpy balance applied at the junction, then the temperature profile of the main wellbore above the junction was calculated.

\begin{tabular}{|c|c|}
\hline Geothermal Gradient ${ }^{\circ} \mathrm{F} / \mathrm{ft}$ & 0.027 \\
\hline $\mathrm{C}_{\mathrm{po}}, \mathrm{Btu} / \mathrm{lbm}^{0} \mathrm{~F}$ & 0.49 \\
\hline Wellbore diameter, in & 7.5 \\
\hline Outside Casing diameter, in & 5.5 \\
\hline Inside Casing diameter, in & 5.05 \\
\hline Thermal Conductivity of cement, Btu/D ft ${ }^{\circ} \mathrm{F}$ & 96.5 \\
\hline Thermal Conductivity of earth, Btu/D ft ${ }^{\circ} \mathrm{F}$ & 33.6 \\
\hline Flow rate of oil, STB/D & 200 \\
\hline${ }^{\circ} \mathrm{API}$ & 35 \\
\hline Oil Gravity & 0.85 \\
\hline Oil Density, $\mathrm{lbm} / \mathrm{ft}^{3}$ & 53.03 \\
\hline Temperature at $Z=0,{ }^{\circ} \mathrm{F}$ & 237.2 \\
\hline Radious from lateral to vertical, $\mathrm{ft}$ & 1500 \\
\hline The dimensionless time function & 2.51 \\
\hline Oil Flow Rate, Ibm/sec & 0.69 \\
\hline Overall Heat Transfer Coefficient, Btu/lbm ${ }^{0} \mathrm{~F} \mathrm{ft}{ }^{2}$ & 1479.54 \\
\hline Coefficient $\mathrm{A}, \mathrm{ft}$ & 343.55 \\
\hline
\end{tabular}

Table 5.1 Properties used in calculation of temperature profiles 


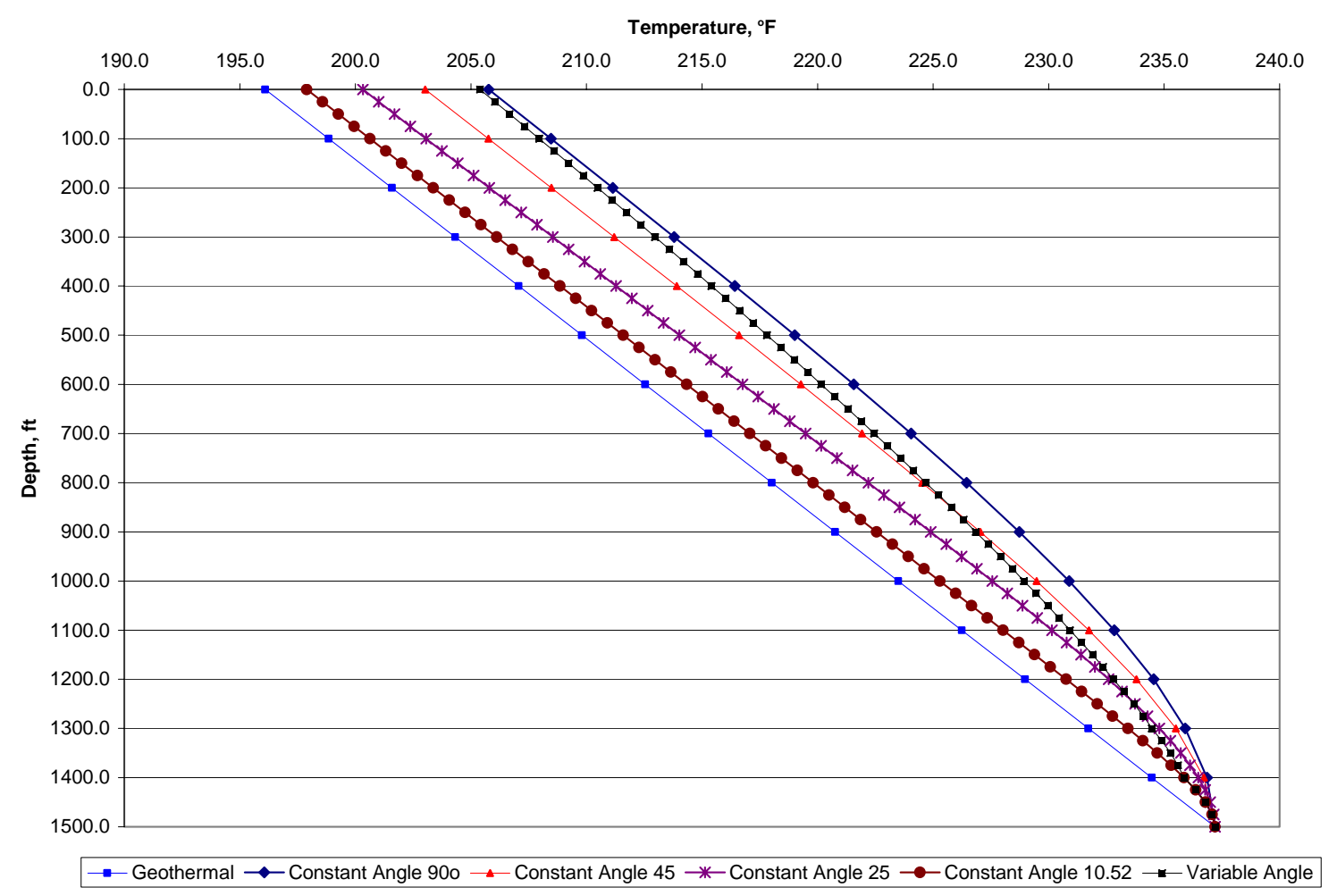

Fig. 5.4 Comparison of predicted temperature profile with constant angles and variable angle along build section

\section{$\underline{\text { Same Heat Capacities and Flow Rates for Both Streams }}$}

Temperature profiles with same heat capacities and flow rates for each lateral (condition1: constant angle, condition 2: variable angle) were calculated using the data given in Table 5.2. The results for this case are shown in Fig. 5.5, where it can be seen that both laterals reach the same value of fluid temperature before mixing because of similar conditions and after mixing the difference between geothermal and fluid temperature increases because of the increase in flow rate after mixing both streams. 


\begin{tabular}{|c|c|c|c|}
\hline & Condition 1 & Condition 2 & Junction (Condition 3) \\
\hline Geothermal Gradient ${ }^{\circ} \mathrm{F} / \mathrm{ft}$ & 0.03 & 0.03 & 0.03 \\
\hline $\mathrm{C}_{\mathrm{p} 1}, \mathrm{Btu} / \mathrm{lbm}^{0} \mathrm{~F}$ & 0.49 & 0.49 & 0.49 \\
\hline Wellbore diameter, in & 7.5 & 7.5 & 7.5 \\
\hline Outside Casing diameter, in & 5.5 & 5.5 & 5.5 \\
\hline Inside Casing diameter, in & 5.05 & 5.05 & 5.05 \\
\hline Thermal Conductivity of cement, Btu/D ft ${ }^{\circ} \mathrm{F}$ & 96.5 & 96.5 & 96.5 \\
\hline Thermal Conductivity of earth, Btu/D ft ${ }^{\circ} \mathrm{F}$ & 33.6 & 33.6 & 33.6 \\
\hline Flow rate of oil, STB/D & 100 & 100 & 200 \\
\hline${ }^{\circ} \mathrm{API}$ & 35 & 35 & 35 \\
\hline Oil Gravity & 0.85 & 0.85 & 0.85 \\
\hline Oil Density, lbm/ft ${ }^{3}$ & 53.03 & 53.03 & 53.03 \\
\hline Temperature at $\mathrm{Z}=0,{ }^{\circ} \mathrm{F}$ & 237.2 & 237.2 & 237.2 \\
\hline Radious from lateral to vertical, $\mathrm{ft}$ & 1500 & 1500 & 1500 \\
\hline The dimensionless time function & 2.51 & 2.51 & 2.51 \\
\hline $\begin{array}{c}\text { Oil Flow Rate, } \mathrm{lbm} / \mathrm{sec} \\
\end{array}$ & 0.34 & 0.34 & 0.69 \\
\hline Overall Heat Transfer Coefficient, Btu/lbm ${ }^{\circ} \mathrm{F} \mathrm{ft}{ }^{2}$ & 1479.54 & 1479.54 & 1479.54 \\
\hline Coefficient $\mathrm{A}$ for Casing Flow, $\mathrm{ft}$ & 179.17 & 179.17 & 358.33 \\
\hline Temperature at the junction, ${ }^{\circ} \mathrm{F}$ & & & 200.87 \\
\hline
\end{tabular}

Table 5.2 Properties used in calculation of temperature profiles for two laterals with same heat capacities and flow rates mixed at junction

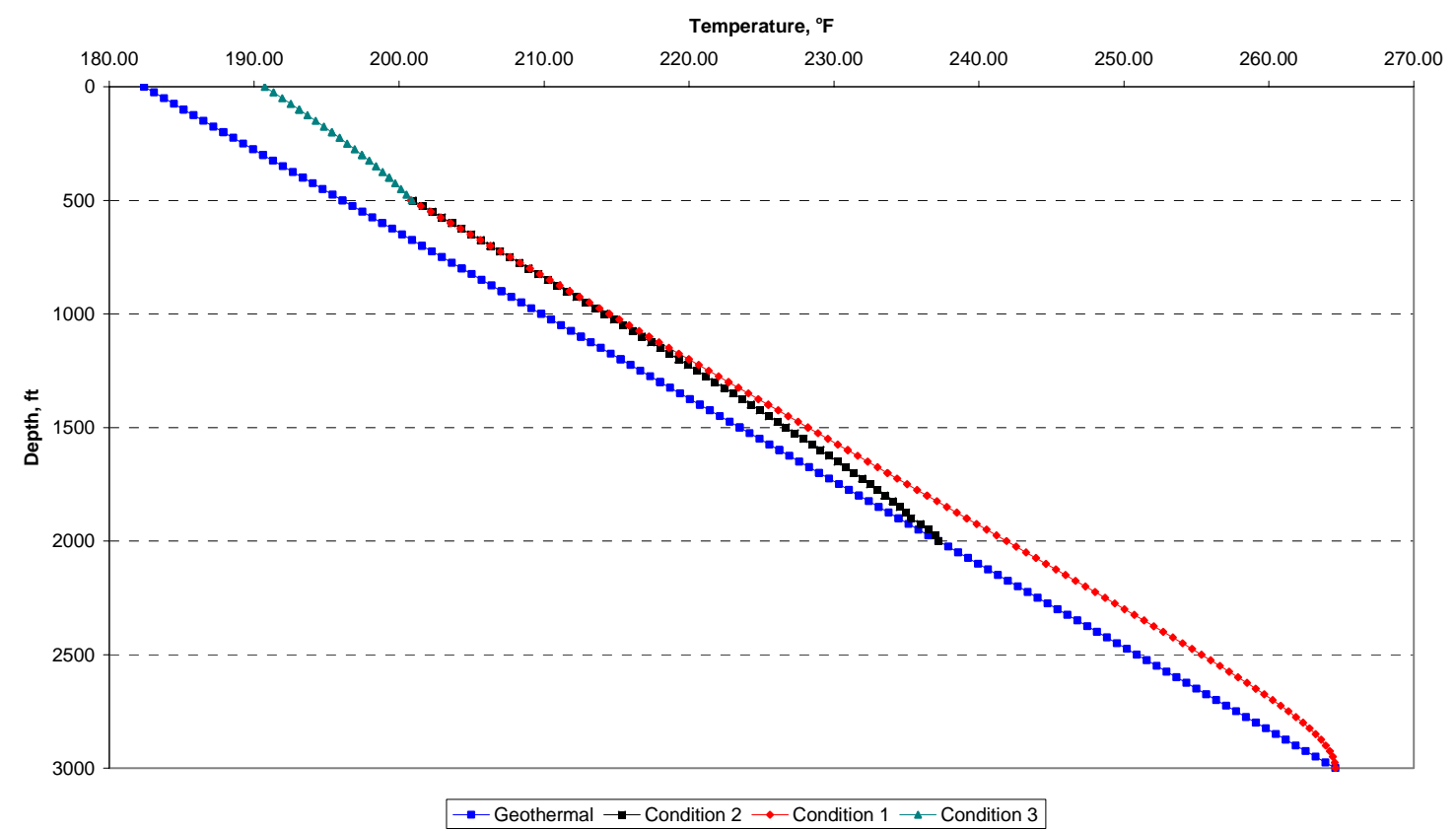

Fig. 5.5 Temperature profiles for two laterals with same heat capacities and flow rates mixed at junction 


\section{Different Heat Capacities and Flow Rates for Both Streams}

Temperature profiles with different heat capacities and flow rates for each lateral (condition1: constant angle, condition 2: variable angle) were calculated using the data given in Table 5.3. The results for this case are shown in Fig. 5.6, where it can be seen that both laterals reach different values of fluid temperature at the moment of mixing because of differences in heat capacities and flow rates for each one. In this case the mixed stream temperature is between each temperature stream and then, the mixed stream presents a bigger difference between geothermal and fluid temperature.

\begin{tabular}{|c|c|c|c|}
\hline & Condition 1 & Condition 2 & Junction (Condition 3) \\
\hline Geothermal Gradient ${ }^{\circ} \mathrm{F} / \mathrm{ft}$ & 0.03 & 0.03 & 0.03 \\
\hline $\mathrm{C}_{\mathrm{p} 1}, \mathrm{Btu} / \mathrm{lbm}^{\circ} \mathrm{F}$ & 0.6 & 0.49 & 0.55 \\
\hline Wellbore diameter, in & 7.5 & 7.5 & 7.5 \\
\hline Outside Casing diameter, in & 5.5 & 5.5 & 5.5 \\
\hline Inside Casing diameter, in & 5.05 & 5.05 & 5.05 \\
\hline Thermal Conductivity of cement, Btu/D ft ${ }^{\circ} \mathrm{F}$ & 96.5 & 96.5 & 96.5 \\
\hline Thermal Conductivity of earth, Btu/D ft ${ }^{\circ} \mathrm{F}$ & 33.6 & 33.6 & 33.6 \\
\hline Flow rate of oil, STB/D & 300 & 200 & 500 \\
\hline${ }^{\circ} \mathrm{API}$ & 35 & 35 & 35 \\
\hline Oil Gravity & 0.85 & 0.85 & 0.85 \\
\hline Oil Density, $\mathrm{Ibm} / \mathrm{ft}^{3}$ & 53.03 & 53.03 & 53.03 \\
\hline Temperature at $\mathrm{Z}=0,{ }^{\circ} \mathrm{F}$ & 237.2 & 237.2 & 237.2 \\
\hline Radious from lateral to vertical, $\mathrm{ft}$ & 1500 & 1500 & 1500 \\
\hline The dimensionless time function & 2.51 & 2.51 & 2.51 \\
\hline Oil Flow Rate, Ibm/sec & 1.03 & 0.69 & 1.72 \\
\hline Overall Heat Transfer Coefficient Btu/lbm ${ }^{\circ} \mathrm{F} \mathrm{ft}^{2}$ & 1479.54 & 1479.54 & 1479.54 \\
\hline Coefficient A for Casing Flow, ft & 664.95 & 358.33 & 1023.28 \\
\hline Temperature at the junction, ${ }^{\circ} \mathrm{F}$ & & & 210.48 \\
\hline
\end{tabular}

Table 5.3 Properties used in calculation of temperature profiles along build section and junction with different heat capacities and flow rates 


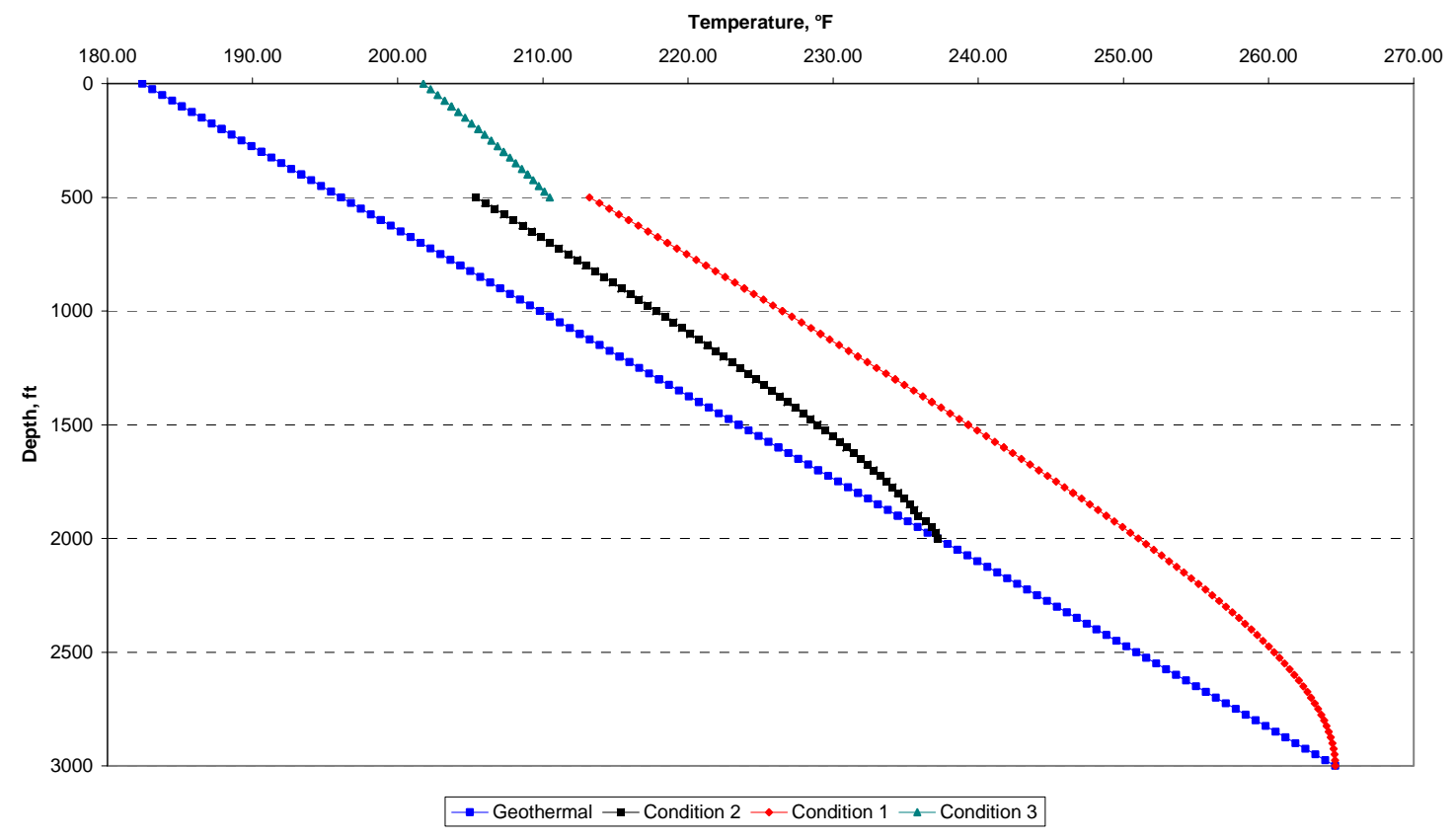

Fig. 5.6 Predicted temperature profiles for two laterals with different heat capacities and flow rates mixed at junction

\subsection{Conclusions of Build Section Model}

Calculating the temperature profile with variable angle along the build section considering that the trajectory follows the path of a curve (a quarter of a circle) gives realistic results that can improve the development of a forward model of wellbore fluids temperature and pressure. For single phase flow at every junction in a multilateral well, commingling of fluid streams with different temperatures can be modeled using the mixing method applying an enthalpy balance at the junction. 


\section{Conclusions}

The work to date on the forward model of temperature and pressure behavior in complex wells has been divided into the three physical parts of the system: the reservoir, the producing laterals, and the build sections connecting laterals and the main wellbore. Conclusions regarding each part of this system are:

Reservoir: Both Joule-Thomson expansion and frictional heating in the near wellbore region can create temperature changes of several degrees, though often in opposite directions (Joule-Thomson cooling and frictional heating). This level of temperature change is easily detectable with current smart well hardware.

Lateral flow: Thermal effects in the wellbore itself are small and may not be detectable in many cases. However, when a change in inflow conditions along the lateral occurs, noticeable and measurable inflections occur in the wellbore temperature profile. Coupling of the lateral model with the reservoir model will allow us to explore this in detail.

Build section: The temperature in the build section can be predicted by adapting the Ramey Equation to the complex , variable-inclination geometry of a typical build section. Mixing effects at junctions are sometimes very pronounced, suggesting that flow rate from individual laterals may be obtainable from the temperature in the vicinity of the junction. This effect depends strongly on the trajectories of the laterals. 


\section{Nomenclature}

\begin{tabular}{|c|c|}
\hline$A$ & relaxation distance \\
\hline$C_{p}$ & specific heat capacity \\
\hline $\overrightarrow{\mathbf{e}}$ & combined energy flux vector \\
\hline$E_{t}$ & total energy \\
\hline$f$ & friction factor \\
\hline$f(t)$ & time function \\
\hline$g$ & gravity acceleration \\
\hline$g_{c}$ & conversion factor, $32.17 \mathrm{lbm}-\mathrm{ft} / \mathrm{lbf}_{-\mathrm{s}^{2}}$ \\
\hline$g_{G}$ & geothermal gradient \\
\hline$h$ & reservoir thickness \\
\hline$H$ & enthalpy \\
\hline$J$ & productivity index \\
\hline$J$ & mechanical equivalent of heat \\
\hline$k$ & Permeability \\
\hline$k_{\text {cem }}$ & thermal conductivity of cement \\
\hline$k_{e}$ & thermal conductivity of earth or formation \\
\hline$K_{T t}$ & Total thermal conduction coefficient \\
\hline$L$ & total measure well depth \\
\hline$N_{\mathrm{Re}}$ & Reynolds number \\
\hline$N_{\mathrm{Re}, w}$ & wall Reynolds number \\
\hline$p$ & pressure \\
\hline q & flow rate \\
\hline $\overrightarrow{\mathbf{q}}$ & heat flux \\
\hline$Q$ & heat transfer rate per unit length of wellbore \\
\hline$R$ & radius \\
\hline$R$ & distance from lateral and main wellbore \\
\hline$t$ & time \\
\hline$T$ & temperature \\
\hline$T_{w}$ & temperature of fluid entering a wellbore \\
\hline$T_{e i}$ & formation temperature at initial condition \\
\hline$T_{\text {eibh }}$ & static formation temperature at the bottom hole \\
\hline $\overrightarrow{\mathbf{u}}$ & Darcy velocity \\
\hline$U$ & internal energy \\
\hline$U$ & heat transfer coefficient \\
\hline$v$ & velocity \\
\hline$\hat{V}$ & specific volume \\
\hline$w$ & wellbore mass flow rate \\
\hline $\mathrm{W}$ & Work \\
\hline $\mathrm{Y}$ & Length of reservoir \\
\hline
\end{tabular}




\begin{tabular}{|c|c|}
\hline$z$ & variable well depth from surface \\
\hline$\alpha$ & momentum correction factor \\
\hline$\beta$ & thermal expansion coefficient \\
\hline$\delta_{i j}$ & Kronecker delta \\
\hline$\varepsilon$ & relative roughness of pipe \\
\hline$\mu$ & viscosity \\
\hline $\overrightarrow{\boldsymbol{\pi}}$ & molecular momentum flux vector \\
\hline$\rho$ & density \\
\hline$\phi$ & Porosity \\
\hline$\theta$ & angle from horizontal \\
\hline$\tau$ & dummy variable of integration \\
\hline $\overrightarrow{\overline{\boldsymbol{\tau}}}$ & stress shear tensor \\
\hline \multicolumn{2}{|c|}{ Subscripts } \\
\hline$c i$ & casing inside \\
\hline $\mathrm{CO}$ & casing outside \\
\hline cem & cement \\
\hline$e$ & earth, formation \\
\hline$I$ & inflow \\
\hline$i$ & cell number \\
\hline$m$ & mixed stream \\
\hline$O$ & oil \\
\hline$r$ & r-coordinate \\
\hline$R$ & reservoir \\
\hline$t i$ & tubing inside \\
\hline to & tubing outside \\
\hline$x$ & $\mathrm{x}$-coordinate \\
\hline$w$ & well location \\
\hline$w b$ & wellbore \\
\hline$\theta$ & $\theta$-coordinate \\
\hline 1 & stream 1 \\
\hline 2 & stream 2 \\
\hline \multicolumn{2}{|c|}{ Superscripts } \\
\hline$o$ & original \\
\hline \multicolumn{2}{|c|}{ Overlines } \\
\hline $\bar{X}$ & average \\
\hline$\hat{X}$ & per unit mass \\
\hline $\overrightarrow{\mathbf{X}}$ & vector \\
\hline $\overrightarrow{\overrightarrow{\mathbf{X}}}$ & tensor \\
\hline
\end{tabular}




\section{References}

1. Al-Hadhrami, A.K., Elliott, L., and Ingham, D.B.,: “A New Model for Viscous Dissipation in Porous Media Across a Range of Permeability Values," Transport in Porous Media 53, Netherlands, 2003.

2. Alves, I.N., Alhanatl, F.J.S. and Shoham, O.; “A Unified Model for Predicting Flowing Temperature Distribution in Wellbores and Pipelines”, SPE 20632, 1992

3. Barua, S.: "Computation of Heat Transfer in Wellbores With Single and Dual Completions," paper SPE 22868 presented at the $66^{\text {th }}$ Annual Technical Conference and Exhibition of the Society of Petroleum Engineers held in Dallas, TX, October 6-9 1991.

4. Bird, R. B., Stewart, W.E., and Lightfoot, E.N.; “Transport Phenomena”, John Wiley and Sons, New York, NY, 2002.

5. Bowman, W.J., and Hitchcock, J.; "Transient, Compressible Heat-pipe Vapor Dynamics”, Proc. 25 ${ }^{\text {th }}$ ASME Natn. Heat Transfer Conf., Houston, Texas, pp. 329-337, 1988.

6. Brill, J.P., Mukherjee, H. : “Multiphase Flow in Wells,” Monograph volume 17, SPE Henry L. Doherty series, Society of Petroleum Engineers, Richardson, TX, 1999.

7. Brown, G., Storer, D., McAllister, K., Al-Asimi, M., and Raghavan, K. : “Monitoring Horizontal Producers and Injectors During Cleanup and Production Using Fiber-OpticDistributed Temperature Measurements" paper SPE 84379 presented at the SPE Annual Technical Conference and Exhibition, Denver, CO, 5-8 October, 2003.

8. Butler, R.M. : “Horizontal Wells for the Recovery of Oil, Gas, and Bitumen”, The Petroleum Society of the Canadian Institute of Mining, Metallurgy and Petroleum, Calgary, Canada, 1994.

9. Chen, W., Zhu, D., Hill, A.D.: “A Comprehensive Model of Multilateral Well Deliverability," paper SPE 64751 presented at the SPE International Oil and Gas Conference and Exhibition in China held in Beijing, China, 7-10 November 2000.

10. Economides, M.J., Hill, A.D., and Ehilig- Economides, C.; "Petroleum Production Systems”, Prentice Hall Inc., New Jersey, 1994

11. Furui, K., Zhu, D., and Hill, A.D. : “A Rigorous Formation Damage Skin Factor and Reservoir Inflow Model for a Horizontal Well” paper SPE 74698 presented at the SPE International Symposium and Exhibition on Formation Damage Control, Lafayette, LA, 20-21 February, 2002. 
12. Gringarten, A. C., and Ramey, H. J. Jr.: “The Use of Source and Green's Functions in Solving Unsteady-Flow Problem in Reservoirs”, paper SPE 3818, Society of Petroleum Engineers Journal, October, 1973.

13. Hasan, A.R. and Kabir, C.S.: “Aspects of Wellbore Heat Transfer During Two-Phase Flow,” SPEPF 211, August 1994.

14. Hasan, A.R. and Kabir, C.S.: "Fluid Flow and Heat Transfer in Wellbores," Society of Petroleum Engineers, Richardson, TX, 2002.

15. Hill, A.D.; "Production Logging-Theoretical and Interpretive Elements", Society of Petroleum Engineers Inc., Richardson, TX, 1990

16. Ingham,D.B., Pop, I., and Cheng, P.: “Combined Free and Forced Convection in a Porous Medium Between Two Vertical Walls with Viscous Dissipation”, Transport in Porous Media 5, Netherlands, 1990.

17. Lake, L. W.: “Enhanced Oil Recovery”, Prentice Hall, Saddle River, NJ, 1989.

18. Li, H., Zhu, D., Lake, L.W., and Hill, A.D.; ” A New Method to Interpret Two-Phase Profiles from Temperature and Flowmeter Log”, SPE 56793, 1999

19. Maubeuge, F., Didek, M., Beardsell, M.B., Arquis, E., Bertrand, O., and Caltagirone, J.P.: “MOTHER: A Model for Interpreting Thermometrics”, paper SPE 28588 presented at the Society of Petroleum Engineers $69^{\text {th }}$ Annual Technical Conference and Exhibition, New Orleans, LA, 25-28 September, 1994.

20. Ouyang, L.B., Arbabi, S., and Aziz, K.; “A Single-Phase Wellbore-Flow Model for Horizontal, Vertical, and Slanted Wells”, SPE Journal, June, 1998

21. Ouyang, L.B., and Aziz, K.: "A Simplified Approach to Couple Wellbore Flow and Reservoir Inflow for Arbitrary Well Configurations," paper SPE 48936 presented at the SPE Annual Technical Conference and Exhibition, New Orleans, LA, 27-30 September, 1998.

22. Petricola, M.F.J. and Watfa, M.: "Multiwell Application of Downhole Temperature Profiles for Crossflow Analysis,” paper SPE 25630 presented at the SPE Middle East Oil Technical Conference and Exhibition held in Bahrain, 3-6 April 1993.

23. Ramey, H.J., Jr.: “Wellbore Heat Transmission,” Journal of Petroleum Technology, Trans. AIME volume 225, pp. 427-435, April 1962.

24. Sagar, R.K., Dotty, D.R., and Schmidt, Z.: "Predicting Temperature Profiles in a Flowing Well,” paper SPE 19702, November 1991.

25. Sensornet Limited, www.sensornet.co.uk, 2004. 
26. Steffensen, R.J., and Smith, R.C. : "The Importance of Joule-Thomson Heating (or Cooling) in Temperature Log Interpretation," paper SPE 4636 presented at the $48^{\text {th }}$ Annual Fall Meeting of the Society of Petroleum Engineers of AIME, Las Vegas, NV, 30 September- 3 October, 1973.

27. Tolan, M., Boyle, M., and Williams, G. : “The Use of Fiber-Optic Distributed Temperature Sensing and Remote Hydraulically Operated Interval Control Valves for the Management of Water Production in the Douglas Field," paper SPE 71676 presented at the 2001 SPE Annual Technical Conference and Exhibition, New Orleans, LA, 30 September-3 October, 2001.

28. White, F. M.; “Fluid Mechanics”. McGraw-Hill Book Co., New York, NY, 1986 
$\underline{\text { Appendix A }}$ : Temperature Model for Slightly Compressible Fluid

As can be seen in Fig. 3.3, there are two flow regions, radial and linear. First consider linear flow region. The pressure relationship is described by Darcy's law as:

$u_{y}=-\frac{k}{\mu} \frac{d p}{d y}$

And the above equation can be expressed for slightly compressible fluid as:

$\frac{q}{2 L h}=-\frac{k}{\mu} \frac{d p}{d y}$

The energy balance is expressed in the following form:

$\rho C_{p} \overrightarrow{\mathbf{u}} \cdot \nabla T+\overrightarrow{\mathbf{u}} \cdot \nabla p-\beta T \overrightarrow{\mathbf{u}} \cdot \nabla p+\nabla \cdot p \overrightarrow{\mathbf{u}}-\nabla \cdot \overrightarrow{\mathbf{K}}_{\mathrm{Tt}} \nabla T=0$

In one dimensional Cartesian coordinate (y-direction), the equation becomes

$$
\rho C_{p} u_{y} \frac{d T}{d y}-\beta T u_{y} \frac{d p}{d y}+2 u_{y} \frac{d p}{d y}-K_{T t} \frac{d^{2} T}{d y^{2}}=0
$$

Substituting Eq. A.2 into Eq. (A.4) and rearranging gives

$$
\frac{d^{2} T}{d y^{2}}-\frac{\rho C_{p}}{K_{T t}}\left(\frac{q}{2 h L}\right) \frac{d T}{d y}-\frac{\beta \mu}{k K_{T t}}\left(\frac{q}{2 h L}\right)^{2} T+\frac{2 \mu}{k K_{T t}}\left(\frac{q}{2 h L}\right)^{2}=0
$$

Solving the second order ordinary differential equation gives

$$
T=c_{1} e^{m_{1} y}+c_{2} e^{m_{2} y}+\frac{2}{\beta}
$$

where

$$
m_{1}=\frac{q}{4 h L}\left[\frac{\rho C_{p}}{K_{T t}}+\sqrt{\left(\frac{\rho C_{p}}{K_{T t}}\right)^{2}+\frac{4 \beta \mu}{k K_{T t}}}\right]
$$

and

$$
m_{2}=\frac{q}{4 h L}\left[\frac{\rho C_{p}}{K_{T t}}-\sqrt{\left(\frac{\rho C_{p}}{K_{T t}}\right)^{2}+\frac{4 \beta \mu}{k K_{T t}}}\right]
$$


Applying boundary conditions, $T=T_{0}$ at $y=\frac{Y}{2}$ and the effective heat transfer of radial flow and linear flow are equal at $\frac{h}{2}$, i.e. $\left(\frac{d T}{d r}\right)_{\text {radial }}=\left(\frac{d T}{d y}\right)_{\text {linear }}$ at $y=\frac{h}{2}$, to evaluate the integration constants $\left(\mathrm{c}_{1}, \mathrm{c}_{2}\right) . T_{0}$ is geothermal temperature at outer boundary. Then, $\mathrm{c}_{1}$ and $\mathrm{c}_{2}$ are determined as below

$$
T_{e}=c_{1} e^{m_{1} h / 2}+c_{2} e^{m_{2} h / 2}+\frac{2}{\beta}
$$

$c_{1}=\frac{(2 / h) m_{r}\left(T_{e}-2 / \beta\right) e^{m_{2} Y / 2}-\left(T_{o}-2 / \beta\right) m_{2} e^{m_{2} h / 2}}{m_{1} e^{m_{1} h / 2+m_{2} Y / 2}-m_{2} e^{m_{2} h / 2+m_{1} Y / 2}}$

$c_{2}=\frac{\left(T_{o}-2 / \beta\right) m_{1} e^{m_{1} h / 2}-(2 / h) m_{r}\left(T_{e}-2 / \beta\right) e^{m_{1} Y / 2}}{m_{1} e^{m_{1} h / 2+m_{2} Y / 2}-m_{2} e^{m_{2} h / 2+m_{1} Y / 2}}$

$$
m_{r}=\frac{q}{4 \pi L}\left[\frac{\rho C_{p}}{K_{T t}}-\sqrt{\left(\frac{\rho C_{p}}{K_{T t}}\right)^{2}+\frac{4 \mu \beta}{k K_{T t}}}\right]
$$

At $y=\frac{h}{2}$ is the boundary of radial flow region, the temperature is

$$
T_{\text {linear at } \frac{h}{2}}=c_{1} e^{m_{1} h / 2}+c_{2} e^{m_{2} h / 2}+\frac{2}{\beta}
$$

By substituting the linear temperature at $\frac{h}{2}$ into the radial solution derived in Appendix B. The temperature profile in the radial flow region is

$T=\frac{2}{\beta}+\left(T_{\text {linear at } \frac{h}{2}}-\frac{2}{\beta}\right)\left(\frac{r}{h / 2}\right)^{\frac{q}{4 \pi L}\left[\frac{\rho C_{p}}{K_{T t}}-\sqrt{\left(\frac{\rho C_{p}}{K_{T t}}\right)+\frac{4 \mu \beta}{k K_{T t}}}\right]}$ 
$\underline{\text { Appendix B }}$ : Temperature Model for Slightly Compressible Fluid in Radial Coordinate System

Consider a steady state radial flow in a homogeneous reservoir with thickness, $h$, as shown in figure below.

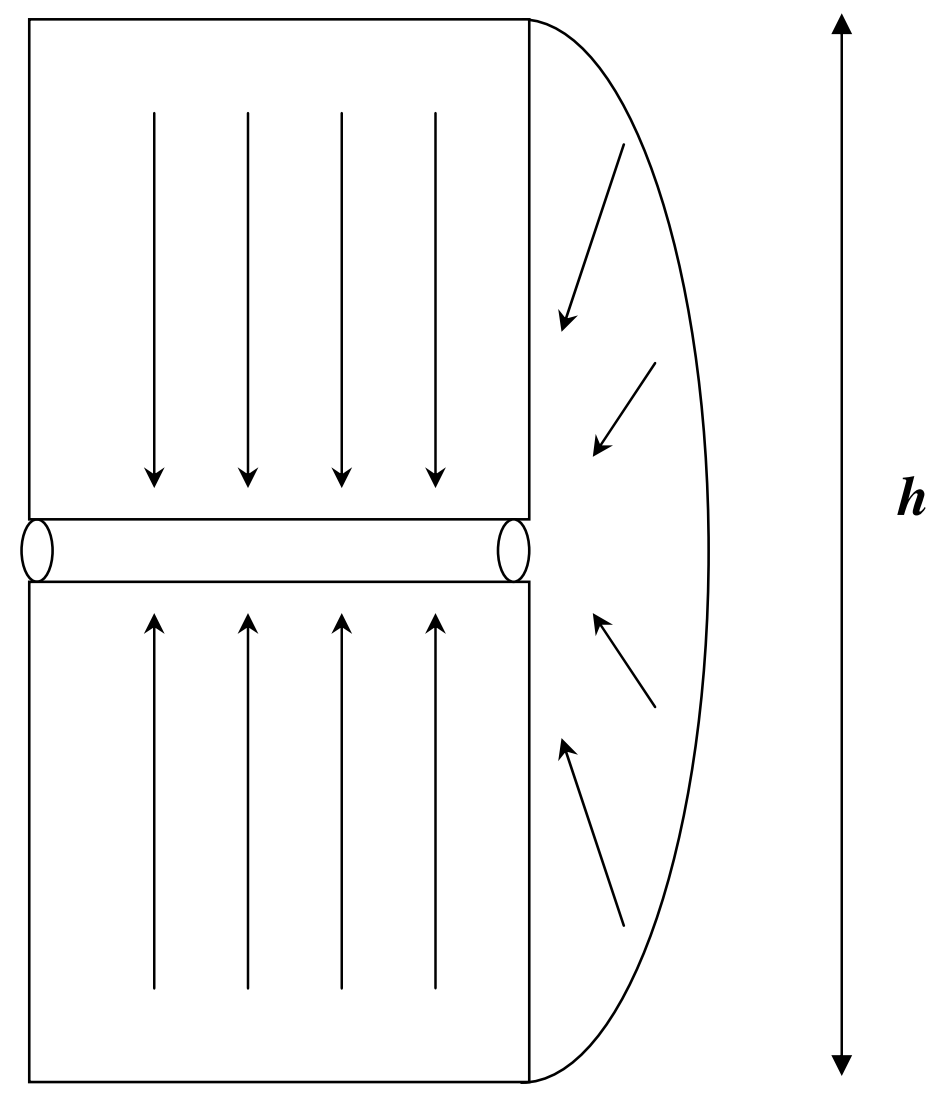

Fig. B.1 Radial flow direction

The pressure relationship is described by Darcy’s law as

$u_{r}=-\frac{k}{\mu} \frac{d p}{d r}$

And Eq. B.1 can be expressed for slightly compressible fluid as:

$\frac{q}{2 \pi r h}=-\frac{k}{\mu} \frac{d p}{d r}$

The energy balance which is derived earlier is expressed in the following form. 
$\rho C_{p} \vec{u} \cdot \vec{\nabla} T+\vec{u} \cdot \vec{\nabla} p-\beta T \vec{u} \cdot \vec{\nabla} p+\mu_{d} \vec{\nabla} \cdot p \vec{u}-\vec{\nabla} \cdot K_{T t} \vec{\nabla} T=0$

In radial coordinate system, the Eq. B.3 becomes

$$
\rho C_{p} u_{r} \frac{d T}{d r}-\beta T u_{r} \frac{d p}{d r}+2 u_{r} \frac{d p}{d r}-K_{T t} \frac{1}{r} \frac{d}{d r}\left(r \frac{d T}{d r}\right)=0
$$

By substituting Eq. B.2 into Eq. B.4 gives

$$
-\frac{2 \pi h K_{T t}}{q} r^{2} \frac{d^{2} T}{d r^{2}}+\left(\rho C_{p}-\frac{2 \pi h K_{T t}}{q}\right) r \frac{d T}{d r}+\frac{\mu q \beta T}{2 \pi k h}-\frac{\mu q}{\pi k h}=0
$$

Solution to this second order differential equation is

$$
T=c_{1} r^{m_{1}}+c_{2} r^{m_{2}}+\frac{2}{\beta}
$$

Where

$$
\begin{aligned}
& m_{1}=\frac{q}{4 \pi h}\left[\frac{\rho C_{p}}{K_{T t}}+\sqrt{\left.\left(\frac{\rho C_{p}}{K_{T t}}\right)^{2}+\frac{4 \mu \beta}{k K_{T t}}\right]}=\right.\text { negative value } \\
& m_{2}=\frac{q}{4 \pi h}\left[\frac{\rho C_{p}}{K_{T t}}-\sqrt{\left(\frac{\rho C_{p}}{K_{T t}}\right)^{2}+\frac{4 \mu \beta}{k K_{T t}}}\right]=\text { positive value }
\end{aligned}
$$

Apply the first boundary condition which is that $T$ is finite as $r$ approaches zero. So, the constant of integration $c_{1}$ must be zero. The Eq. B.6 becomes

$$
T=c_{2} r^{m_{2}}+\frac{2}{\beta}
$$

Appling the second boundary condition which is $T=T_{0}$ at $r=r_{e}$, then $c_{2}$ can be evaluate

$$
c_{2}=\left(T_{0}-\frac{d}{b}\right)\left(\frac{1}{r_{e}}\right)^{m_{2}}
$$

Finally, substituting Eq. B.10 into Eq. B.9 the solution of the differential equation yields 


$$
T=\frac{2}{\beta}+\left(T_{0}-\frac{2}{\beta}\right)\left(\frac{r}{r_{e}}\right)^{\frac{q}{4 \pi h}\left[\frac{\rho C_{p}}{K_{T t}}-\sqrt{\left(\frac{\rho C_{p}}{K_{T t}}\right)^{2}+\frac{4 \mu \beta}{k K_{T t}}}\right]}
$$




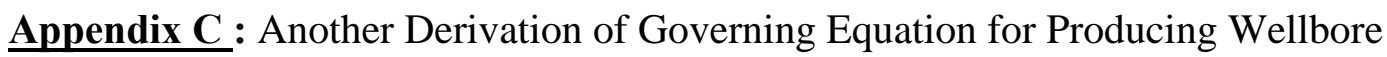

In 2-D cylindrical coordinate, flow equations are given by Tannehill

Mass balance equation

$\frac{\partial \rho}{\partial t}+\frac{\partial\left(\rho v_{x}\right)}{\partial x}+\frac{1}{r} \frac{\partial\left(r \rho v_{r}\right)}{\partial r}=0$

Momentum balance equation

Axial direction

$\frac{\partial\left(\rho v_{x}\right)}{\partial t}+\frac{\partial}{\partial x}\left(\rho v_{x} \cdot v_{x}+p-\tau_{x x}\right)+\frac{1}{r} \frac{\partial}{\partial r} r\left(\rho v_{x} \cdot v_{r}-\tau_{r x}\right)-\rho g \sin \theta=0$

Radial direction

$\frac{\partial\left(\rho v_{r}\right)}{\partial t}+\frac{\partial}{\partial x}\left(\rho v_{x} \cdot v_{r}-\tau_{x r}\right)+\frac{1}{r} \frac{\partial}{\partial r} r\left(\rho v_{r} \cdot v_{r}+p-\tau_{r r}\right)=0$

Viscous stress tensors are defined by Navier-Stokes

$$
\begin{aligned}
& \tau_{x x}=\frac{2}{3} \mu\left(2 \frac{\partial v_{x}}{\partial x}-\frac{\partial v_{r}}{\partial r}-\frac{v_{r}}{r}\right) \\
& \tau_{r r}=\frac{2}{3} \mu\left(2 \frac{\partial v_{r}}{\partial r}-\frac{\partial v_{x}}{\partial x}-\frac{v_{r}}{r}\right) \\
& \tau_{x r}=\mu\left(\frac{\partial v_{x}}{\partial r}+\frac{\partial v_{r}}{\partial x}\right)=\tau_{r x}
\end{aligned}
$$

Energy balance equation

$$
\begin{aligned}
& \frac{\partial E_{t}}{\partial t}+\frac{\partial}{\partial x}\left(u\left(E_{t}+p-\tau_{x x}\right)-v \tau_{x r}+q_{x}\right) \\
& +\frac{1}{r} \frac{\partial}{\partial r}\left(r\left[v\left(E_{t}+p-\tau_{r r}\right)-u \tau_{r x}+q_{r}\right]\right)-\rho u g \sin \theta=0
\end{aligned}
$$

Considering same velocity distribution assumption as 


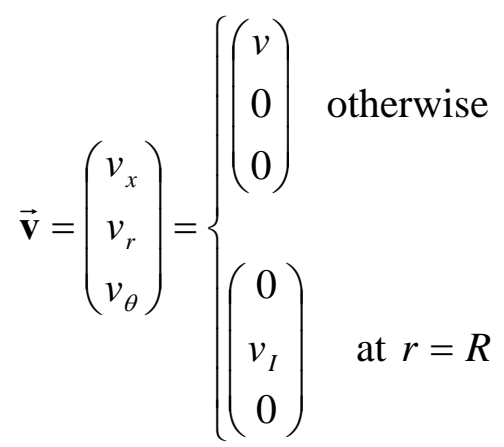

Integrations of those equations are followings;

Mass balance equation is

$r \frac{\partial \rho}{\partial t}+r \frac{\partial\left(\rho v_{x}\right)}{\partial x}+\frac{\partial\left(r \rho v_{r}\right)}{\partial r}=0$

Taking area integral yields

$$
\begin{aligned}
& \int_{0}^{2 \pi} \int_{0}^{R}\left(r \frac{\partial \rho}{\partial t}+r \frac{\partial\left(\rho v_{x}\right)}{\partial x}+\frac{\partial\left(r \rho v_{r}\right)}{\partial r}\right) d r d \theta \\
& =2 \pi\left\{\left(\frac{\partial \rho}{\partial t}+\frac{\partial(\rho v)}{\partial x}\right) \frac{R^{2}}{2}+\int_{0}^{R} \frac{\partial\left(r \rho v_{r}\right)}{\partial r} d r\right\} \\
& =2 \pi\left\{\left(\frac{\partial \rho}{\partial t}+\frac{\partial(\rho v)}{\partial x}\right) \frac{R^{2}}{2}+\int_{0}^{R} \partial\left(r \rho v_{r}\right)\right\} \\
& =2 \pi\left\{\left(\frac{\partial \rho}{\partial t}+\frac{\partial(\rho v)}{\partial x}\right) \frac{R^{2}}{2}-R \rho v_{I}\right\}=0
\end{aligned}
$$

Then, we have

$$
\frac{\partial \rho}{\partial t}=-\frac{\partial(\rho v)}{\partial x}+\frac{2}{R}\left(\rho_{I} v_{I}\right)
$$

Momentum balances are

$$
\frac{\partial\left(\rho v_{x}\right)}{\partial t}+\frac{\partial}{\partial x}\left(\rho v_{x} \cdot v_{x}+p-\tau_{x x}\right)+\frac{1}{r} \frac{\partial}{\partial r} r\left(\rho v_{x} \cdot v_{r}-\tau_{r x}\right)-\rho g \sin \theta=0
$$




$$
\frac{\partial\left(\rho v_{r}\right)}{\partial t}+\frac{\partial}{\partial x}\left(\rho v_{x} \cdot v_{r}-\tau_{x r}\right)+\frac{1}{r} \frac{\partial}{\partial r} r\left(\rho v_{r} \cdot v_{r}+p-\tau_{r r}\right)=0
$$

We are not interested in momentum balance in $r$ direction so only axial direction momentum balance equation will be considered. Taking area integral of $\mathrm{x}$ direction momentum balance gives

$$
\int_{0}^{2 \pi} \int_{0}^{R}\left\{r \frac{\partial\left(\rho v_{x}\right)}{\partial t}+r \frac{\partial}{\partial x}\left(\rho v_{x} \cdot v_{x}+p-\tau_{x x}\right)+\frac{\partial}{\partial r} r\left(\rho v_{x} \cdot v_{r}-\tau_{r x}\right)-r \rho g \sin \theta\right\} d r d \theta=0
$$

Viscous stress shear stresses are from the assumption

$$
\begin{aligned}
& \tau_{x x}=\frac{2}{3} \mu\left(2 \frac{\partial v_{x}}{\partial x}-\frac{\partial v_{r}}{\partial r}-\frac{v_{r}}{r}\right)=\frac{2}{3} \mu\left(2 \frac{\partial v_{x}}{\partial x}-0-0\right)=\frac{4}{3} \mu \frac{\partial v_{x}}{\partial x} \\
& \tau_{x r}=\mu\left(\frac{\partial v_{x}}{\partial r}+\frac{\partial v_{r}}{\partial x}\right)=\mu\left(0+\frac{\partial v_{r}}{\partial x}\right)=\mu \frac{\partial v_{r}}{\partial x} \\
& \int_{0}^{2 \pi}\left\{r \frac{\partial\left(\rho v_{x}\right)}{\partial t}+r \frac{\partial}{\partial x}\left(\rho v_{x} \cdot v_{x}+p-\tau_{x x}\right)+\frac{\partial}{\partial r} r\left(\rho v_{x} \cdot v_{r}-\tau_{r x}\right)-r \rho g \sin \theta\right\} d r d \theta \\
& =\pi R^{2}\left\{\frac{\partial(\rho v)}{\partial t}+\frac{\partial}{\partial x}\left(\rho \alpha v^{2}+p-\frac{4}{3} \mu \frac{\partial v}{\partial x}\right)\right\}+\int_{0}^{2 \pi} \int_{0}^{R}\left\{\frac{\partial}{\partial r} r\left(\rho v_{x} \cdot v_{r}-\tau_{r x}\right)\right\} d r d \theta+\pi R^{2} \rho g \sin \theta \\
& =\pi R^{2}\left\{\frac{\partial(\rho v)}{\partial t}+\frac{\partial}{\partial x}\left(\rho \alpha v^{2}+p-\frac{4}{3} \mu \frac{\partial v}{\partial x}\right)\right\}+2 \pi\left[\int_{0}^{R}\left\{\frac{\partial}{\partial r} r\left(\rho v_{x} \cdot v_{r}\right)\right\} d r-\int_{0}^{R}\left\{\frac{\partial \tau_{r x}}{\partial r}\right\} d r\right]+\pi R^{2} \rho g \sin \theta \\
& =0
\end{aligned}
$$

From the assumption that inflow is perpendicular to the axis at the pipe wall

$$
\begin{aligned}
& \pi R^{2}\left\{\frac{\partial(\rho v)}{\partial t}+\frac{\partial}{\partial x}\left(\rho \alpha v^{2}+p-\frac{4}{3} \mu \frac{\partial v}{\partial x}\right)\right\}+2 \pi\left[\int_{r=0}^{r=R} d\left\{r\left(\rho v_{x} \cdot v_{r}\right)\right\}-\int_{r=0}^{r=R} \partial \tau_{r x}\right]+\pi R^{2} \rho g \sin \theta \\
& =\pi R^{2}\left\{\frac{\partial(\rho v)}{\partial t}+\frac{\partial}{\partial x}\left(\rho \alpha v^{2}+p-\frac{4}{3} \mu \frac{\partial v}{\partial x}\right)\right\}+2 \pi\left[0-\left.r \tau_{r x}\right|_{r=D / 2}\right]+\pi R^{2} \rho g \sin \theta \\
& =\pi R^{2}\left\{\frac{\partial(\rho v)}{\partial t}+\frac{\partial}{\partial x}\left(\rho \alpha v^{2}+p-\frac{4}{3} \mu \frac{\partial v}{\partial x}\right)\right\}-\left.2 \pi R \mu \frac{\partial u}{\partial x}\right|_{r=R}+\pi R^{2} \rho g \sin \theta
\end{aligned}
$$


Wall friction $\tau_{W}$ is defined as

$\tau_{W}=-\left.\mu \frac{\partial u}{\partial r}\right|_{r=R}=\frac{\rho v^{2} f}{2}$

Substituting and dividing $\pi R^{2}$ gives

$$
\frac{\partial(\rho v)}{\partial t}+\frac{\rho v^{2} f}{R}+\frac{\partial\left(\alpha \rho v^{2}+p\right)}{\partial x}-\frac{\partial}{\partial x}\left(\frac{4}{3} \mu \frac{\partial v}{\partial x}\right)+\rho g \sin \theta=0
$$

Integration of energy balance gives

$$
\begin{aligned}
& 2 \pi \int_{0}^{R}\left[r\left\{\frac{\partial E_{t}}{\partial t}+\frac{\partial}{\partial x}\left(v_{x}\left(E_{t}+p-\tau_{x x}\right)-v_{r} \tau_{x r}+q_{x}\right)\right\}+\frac{\partial}{\partial r}\left(r\left[v_{r}\left(E_{t}+p-\tau_{r r}\right)-v_{x} \tau_{r x}+q_{r}\right]\right)-r \rho v_{x} g \sin \theta\right] d r \\
& =\pi R^{2}\left[\frac{\partial E_{t}}{\partial t}+\frac{\partial}{\partial x}\left\{v\left(E_{t}+p-\frac{4}{3} \mu \frac{\partial v}{\partial x}\right)+q_{x}\right\}\right]+\int_{0}^{R} \frac{\partial}{\partial r}\left(r\left[v_{r}\left(E_{t}+p-\tau_{r r}\right)-v_{x} \tau_{r x}+q_{r}\right]\right) d r-\pi R^{2} \rho v g \sin \theta
\end{aligned}
$$

Neglecting viscous shear of $\mathrm{r}, \tau_{r r}=0$, and $\left(v_{x} \tau_{r x}\right)_{r=R}=0$ yield

$$
\begin{aligned}
& \pi R^{2}\left[\frac{\partial E_{t}}{\partial t}+\frac{\partial}{\partial x}\left\{v\left(E_{t}+p-\frac{4}{3} \mu \frac{\partial v}{\partial x}\right)+q_{x}\right\}\right] \\
& -\pi R\left\{v_{I}\left(\left(E_{t}\right)_{I}+p_{I}\right)+q_{I}\right\}-\pi R^{2} \rho v g \sin \theta=0
\end{aligned}
$$

Then, we have

$$
\begin{aligned}
& \frac{\partial E_{t}}{\partial t} \\
& =-\frac{\partial}{\partial x}\left\{v\left(E_{t}+p-\frac{4}{3} \mu \frac{\partial v}{\partial x}\right)+q_{x}\right\}+\frac{2}{R}\left\{v_{I}\left(\left(E_{t}\right)_{I}+p_{I}\right)+q_{I}\right\}+\pi R^{2} \rho v g \sin \theta
\end{aligned}
$$

Obtained Eqs. C.10, C.17 and C.19 are the same Eqs. derived by using macroscopic method. 